\title{
Approved and experimental small-molecule oncology kinase inhibitor drugs: a mid-2016 overview
}

Peter M. Fischer

School of Pharmacy and Centre for Biomolecular Sciences, University of Nottingham, Nottingham NG7 2RD, UK

Abstract. Kinase inhibitor research is a comparatively recent branch of medicinal chemistry and pharmacology and the first small-molecule kinase inhibitor, imatinib, was approved for clinical use only 15 years ago. Since then, 33 more kinase inhibitor drugs have received regulatory approval for the treatment of a variety of cancers and the volume of reports on the discovery and development of kinase inhibitors has increased to an extent where it is now difficult -even for those working in the field- easily to keep an overview of the compounds that are being developed, as currently there are 231 such compounds, targeting 38 different protein and lipid kinases (not counting isoforms), in clinical use or under clinical investigation. The purpose of this review is thus to provide an overview of the biomedical rationales for the kinases being targeted on the one hand, and the design principles, as well as chemical, pharmacological, pharmaceutical, and toxicological kinase inhibitor properties, on the other hand. Two issues that are especially important in kinase inhibitor research, target selectivity and drug resistance, as well as the underlying structural concepts, are discussed in general terms and in the context of relevant kinases and their inhibitors.

Keywords: cancer, oncology, kinase inhibitor, experimental drug, drug design, clinical trials

Contact: Professor Peter Fischer, Centre for Biomolecular Sciences, University of Nottingham, University Park, Nottingham NG7 2RD, UK. Phone +44 1158466 242. Email peter.fischer@nottingham.ac.uk 


\section{Introduction}

Kinases are now one of the most important drug development target classes in several therapeutic indications, but until very recently all kinase inhibitors that were approved for clinical use were anticancer drugs, with the exception of fasudil, a (diazepanesulfonyl)isoquinoline ROCK inhibitor (for protein kinase nomenclature refer to Table S1). ${ }^{1}$ Fasudil has been approved for some years in Japan for the treatment of certain cardiovascular indications ${ }^{2}$ but even this agent -and ROCK inhibitors in general- may in fact also have potential uses in oncology. ${ }^{3}$ However, the situation changed with the U.S. Food and Drug Administration (FDA) approval in 2012 of the pyrrolopyrimidine JAK inhibitor tofacitinib for the treatment of rheumatoid arthritis. ${ }^{4}$ Again JAK inhibitors are also relevant in cancer, as we shall see later. At the time it was expected that the first kinase inhibitor approval outside oncology would act as a watershed and that pharmaceutical companies would accelerate kinase inhibitor development for a range of different therapeutic indications. In fact kinase inhibitors are undergoing clinical development in inflammatory, cardiovascular, neurodegenerative, liver, and metabolic diseases ${ }^{5}$ but since the approval of tofacitinib there has been only one additional approval outside oncology: nintedanib, a multi-targeted kinase inhibitor (MTKI), in idiopathic pulmonary fibrosis (IPF). ${ }^{6}$ Interestingly this compound was also approved in the European Union for lung cancer patients with advanced adenocarcinoma after first-line chemotherapy. Here we shall confine our discussion to kinase inhibitors in oncology, not only approved agents (Figure 1), but all compounds currently undergoing clinical development.

According to an analysis of new anticancer therapeutics that entered the clinic during the period 1990-2006, kinase inhibition was the most prevalent mode of action of experimental agents, whereas previously interference with DNA replication was the most common mechanism. ${ }^{7}$ A different analysis of new clinical oncology agents during a similar period (19952007 ) noted that while clinical development attrition was high (82\%) overall, kinase inhibitors performed significantly better ( $53 \%$ attrition). ${ }^{8}$

Only a few years ago it was easy for those working in the field to keep track of the kinase inhibitor oncology drugs under development but the sheer number of agents that have recently entered clinical studies now renders this difficult. The main purpose of the present review is therefore to provide an up-to-date summary of some of approved and experimental (as verified in relevant publicly accessible literature, corporate disclosures, and https://clinicaltrials.gov/) kinase inhibitor cancer drugs. Salient points regarding kinase inhibitor targets, selectivity, medicinal chemistry, pharmacology, and toxicology will be discussed. Biological agents, including antibodies and antisense oligonucleotides, are not included but the focus is on small-molecule agents. 


\section{Selectivity}

Following the success with the first approved small-molecule kinase inhibitor anticancer drug, imatinib (Figure 1), ${ }^{9}$ especially in the treatment of chronic myelogenous leukaemia $(\mathrm{CML})$, highly specific targeted kinase inhibitors rapidly became a desirable goal in oncology drug discovery. However, demonstration of clear-cut clinical efficacy has been much more elusive with a range of other experimental kinase inhibitor drugs, and this situation has led to the on-going dispute about selective versus so-called multi-targeted agents.

The question is whether 'magic bullets' are preferable to 'magic shotguns' ${ }^{10}$ and arises not so much because one might be superior to the other, but because upon closer examination even the most carefully designed small-molecule kinase inhibitors often inadvertently turn out to be multi-targeted. In the early days of kinase inhibitor development it was not possible to profile the kinase selectivity of candidate molecules extensively, and unexpected kinase targets were frequently discovered for drugs late in the development process. This situation has changed with the advent of kinase panel ${ }^{11}$ and chemical proteomics $^{12}$ technologies that permit kinome-wide analysis of candidate molecules.

Nevertheless, multi-targeted agents are frequently more efficacious than selective ones, ${ }^{13}$ although this may be due to selective drugs lacking efficacy because they block targets that are inappropriate per se or inappropriate for a specific cancer. Furthermore, network models suggest that partial inhibition of a small number of targets can be more efficient than the complete inhibition of a single target. ${ }^{14}$ Certainly the task of devising a kinase inhibitor that exclusively inhibits a range of predetermined kinases - which may not necessarily be structurally related- would appear to be formidable. Although strategies for the rational design of multi-targeted agents by multiple structure-based design and pharmacophore combination ${ }^{15,16}$ have been described, these have not yet been implemented widely for the design of kinase inhibitors with predetermined multiple selectivity.

Active versus inactive kinases. Because the vast majority of small-molecule kinase inhibitors target the ubiquitous ATP-binding site, target selectivity represents the biggest challenge for kinase inhibitor drug design and development. Protein and lipid kinases catalyse the transfer of the $\mathrm{y}$-phosphoryl group of ATP to their specific macromolecular substrates. This requires an architectural arrangement of the kinase to allow simultaneous binding of both substrates and positioning of the catalytic residues for productive phosphoryl-transfer. For protein kinases this canonical arrangement can be illustrated using PKA as an example (Figure 2). ATP is bound in a cleft between the $\mathrm{N}$ - and C-lobes of the kinase. These lobes are connected by the hinge region, which contains the so-called gatekeeper residue. The adenine portion of ATP forms $\mathrm{H}$-bonds with the main-chain peptide bonds of the two hinge residues distal to the gatekeeper. The activation ( $A$ ) loop adopts a conformation that supports binding of the macromolecular substrate and in which the side 
chain of the Asp residue of the conserved DFG motif is positioned towards the ATP $\mathrm{Y}^{-}$ phosphate (DFG in). Furthermore, the active (i.e. catalytically competent) kinase architecture is characterised by a near-linear arrangement of the regulatory ( $R$ ) spine, which is composed of five residues (R0-R4) that interlock through $\mathrm{H}$-bonding (R0-R1), $\mathrm{CH}-\Pi$ (R1R2-R3), and hydrophobic (R3-R4) interactions. Distortion of the linear spine, which contains residues from both the $\mathrm{N}$ - (R3 and R4) and C-lobes (R0, R1, and R2), is characteristic of inactive kinase architectures and can result from tilting of the kinase lobes about the hinge, by conformational preferences of the A-loop as a result of its phosphorylation status, or movement of the aC-helix or the catalytic (C) loop.

Classification of kinase inhibitors. Kinase inhibitors are classified using various criteria and several classification systems are in use. Here we shall use the integrated system recently proposed by Roskoski: ${ }^{17}$ Type-I inhibitors bind to the active kinase conformation with DFG-Asp in (towards the ATP-binding site), the aC-helix in, and with the R-spine in a linear configuration. Type-I $1 \frac{1}{2}$ inhibitors bind to kinases with an inactive (distorted R-spine) conformation with DFG-Asp in but aC-helix out. Type-II inhibitors also bind to inactive kinase conformations, but with DFG-Asp out. By division of the ATP-binding cleft into a front cleft and a back cleft (see below), type-I and type-II inhibitors can be further separated into $A$ and $B$ subtypes, where subtype $A$ extends into the back cleft and subtype $B$ does not. Allosteric inhibitors of type III bind in the cleft between the small and large kinase lobes but adjacent to the ATP-binding pocket, whereas type-IV inhibitors bind outside of the cleft and the phosphoryl-acceptor region. Allosteric ligands that span two regions of the protein kinase domain are type- $V$ inhibitors. Compounds that form covalent adducts with a kinase are type-VI inhibitors.

$X$-ray crystal structures of complexes with type-I inhibitors generally show an active-like kinase conformation with the inhibitors bound at the same site as ATP but making contacts with kinase residues beyond those involved in binding of ATP. Such inhibitors also frequently recapitulate the hinge $\mathrm{H}$-bonding pattern observed with ATP, using a variety of heterocyclic systems. ${ }^{18}$ Whereas binding of ATP does not usually depend on interactions with the gatekeeper, binding of inhibitors generally does. In type-I inhibitors selectivity arises from interactions of the inhibitor with non-conserved residues at the front (solventexposed; hydrophobic pocket I) and the back (towards gatekeeper; hydrophobic pocket II) of the binding cleft.

Type-II inhibitors are still ATP-competitive and bind to the ATP-binding pocket in a similar manner as type-I inhibitors but they bind to kinases with inactive architectures (disrupted $\mathrm{R}$ spine). These architectures possess altered A-loop conformations, where the DFG Asp residue side chain points away from the ATP $Y$-phosphate (DFG out) and the DFG Phe residue ( $R 2$ ) projects into the ATP-binding site. As a result, the $R$ spine is disrupted and the kinase is not catalytically competent. Such a movement of the R2 side chain opens up 
a new binding pocket (sometimes called the back cleft) beyond the gatekeeper and towards the aC-helix. Type-II inhibitors, which are typically larger than type-I inhibitors, usually extend into this pocket, which differs significantly between kinases.

Selectivity based on kinase inhibition potency. Inhibitor selectivity between kinase pairs is commonly expressed as a ratio (Figure $3 a$; selectivity index $1, S I_{1}$ ) between measures of potency, such as half-maximal inhibition values $\left(\mathrm{IC}_{50}\right)$, inhibition constants $\left(K_{\mathrm{i}}\right)$, or dissociation constants $\left(K_{\mathrm{d}}\right)$. Biochemical kinase activity assays are generally used to determine $\mathrm{IC}_{50}$ values but it is important to remember that selectivity ratios for ATPcompetitive inhibitors based on IC $_{50}$ values can be misleading unless kinase assays are performed at ATP concentrations that give half-maximal reaction velocities for the kinases

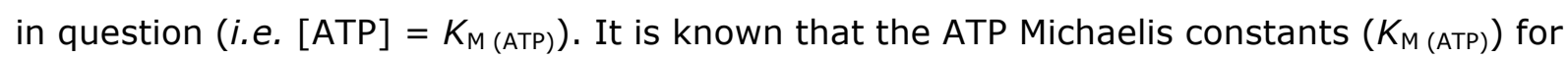
different kinases vary over a range of 3 orders of magnitude from low $\mu \mathrm{M}$ to $\mathrm{mM}$ values. ${ }^{19}$ This is important because in cells, where the ATP concentration is typically as high as 1-5 $\mathrm{mM}$, the potency of an inhibitor against a given kinase is highly dependent on the $K_{\mathrm{M}}$ (ATP) of that kinase. For this reason $K_{\mathrm{i}}$ values are more useful for selectivity measurements, since these take into account $K_{\mathrm{M} \text { (ATP) }}: K_{\mathrm{i}}=\mathrm{IC}_{50} /\left(1+\left([\mathrm{ATP}] / K_{\mathrm{M}(\mathrm{ATP})}\right)\right) .{ }^{20} K_{\mathrm{d}}$ values are typically determined using biophysical binding assays rather than functional kinase assays. Provided assay conditions are similar (concentrations of protein and substrates) then $K_{d}$ and $K_{\mathrm{i}}$ values are also numerically similar.

It is sometimes stated that type-II inhibitors are not ATP competitive and that for this reason the drop-off in cellular potency from biochemical potency is frequently smaller than is the case for type-I inhibitors. ${ }^{21}$ This is not entirely correct, since inactive forms of kinases can still bind ATP, although the difference in $K_{M}$ (ATP) between inactive and activated (phosphorylated) kinase forms can be large. ${ }^{19}$ The ratio of biochemical and cellular potency of a kinase inhibitor will therefore depend on whether it competes with ATP for the active or an inactive state of the target kinase, and in the latter case on the $K_{\mathrm{M} \text { (ATP) }}$ of the inactive state. Regardless of inhibition mode, the relationship between biochemical and cellular potency is of course also affected by factors such as solubility, protein binding, and membrane permeability.

Selectivity based on receptor occupancy. For kinase inhibitor drugs we are interested not so much in selectivity based on differential kinase affinity under equilibrium conditions $\left(S I_{1}\right)$, but in selectivity under physiological conditions in vivo. Here we need to take into account that the magnitude of physiological responses is proportional to the amount of inhibitor bound by a particular kinase over time. To assess true selectivity of kinase inhibitors it is therefore more useful to look at fractional kinase (receptor) occupancy as a function of time $\left(f_{\mathrm{t}}\right)$. Based on this we can formulate a second selectivity index (Figure 3a; $\left.S I_{2}\right)$. 
For a type-I kinase inhibitor that reversibly binds a kinase by mechanism $A, f o_{t}$ depends on ligand concentration and $K_{\mathrm{d}}$ as shown in Figure 3a. To illustrate the relationship between $S I_{1}$ and $S I_{2}$, let us assume that we have kinase inhibitors that all bind a particular on-target kinase with a $K_{\mathrm{d}}$ value of $10 \mathrm{nM}$ but that bind an undesirable (leading to toxicity) off-target kinase with $K_{d}$ values of $50 \mathrm{nM}\left(S I_{1}=5\right), 250 \mathrm{nM}\left(S I_{1}=25\right), 1 \mu \mathrm{M}\left(S I_{1}=100\right)$, and $10 \mu \mathrm{M}$ $\left(S I_{1}=1,000\right)$, respectively. If we assume that these hypothetical kinase inhibitors have identical pharmacokinetic profiles and are dosed repeatedly so as to achieve continuous exposure, measured as the concentration of free drug fraction at steady state $\left(C_{s s f}\right)$, then we can substitute $C_{\mathrm{ssf}}$ for $[\mathrm{L}]_{\mathrm{t}}$ in the expression for $f_{\mathrm{t}}$ and evaluate $S I_{2}$ as a function of $C_{\mathrm{ssf}}$. This analysis (Figure $3 \mathrm{~b}$ ) shows that at $C_{\mathrm{ssf}}=K_{\mathrm{d} \text { (on-target) }}=10 \mathrm{nM}$ we have approximately $50 \%$ on-target receptor occupancy. At that $C_{\text {ssf }}$ value we have $S I_{2}$ values of $3,13,50$, and 500 for the inhibitors with $S I_{1}$ values of $5,25,100$, and 1,000, respectively. Depending on how physiological responses are coupled to on-target kinase occupancy, we may require much higher fo to achieve efficacy. If $90 \%$ occupancy is required ( $f O_{\text {on-target }}=0.9, C_{\text {ssf }}=90$ $\mathrm{nM})$, then the $S I_{2}$ values for our inhibitors are $1.4\left(S I_{1}=2.5\right), 3.4\left(S I_{1}=25\right), 11\left(S I_{1}=\right.$ $50)$, and $100\left(S I_{1}=1,000\right)$, respectively. Depending on how off-target kinase occupancy is coupled to toxicity, the drop-off from $S I_{1}$ to $S I_{2}$ may be smaller or larger but the relationship between selectivity in terms of binding versus occupancy as a function of $C_{\text {ssf }}$ clearly shows that an inhibitor with an $S I_{1}$ value of $<100$ is unlikely to provide a useful margin between on- and off-target activities in vivo.

Selectivity based on residence time. High-affinity kinase inhibitors, especially those of type II, frequently exhibit slow binding kinetics. This is due to induced-fit binding, where the kinase undergoes a time-dependent change from a conformation $\mathrm{R}$ to a conformation $R^{*}$, where the latter has higher affinity for the ligand $L$ than the former (Figure 3a; mechanism $B$ ). Here dissociation (off-rate) and residence time are not defined by a single rate constant as in mechanism $\mathrm{A}$, but by a composite of rate constants, where $k_{4}$ (and sometimes $k_{2}$ ) are limiting for dissociation. For inhibitors that bind kinases in inactive-like conformations, mechanism $C$ is sometimes invoked, especially when these inhibitors selectively bind the kinase in its A-loop non-phosphorylated state. There are currently insufficient kinetic data to distinguish clearly between inhibitors that act by mechanisms $B$ and C, however. ${ }^{22}$

In any case, the situation often arises where an inhibitor exhibits kinase residence time that is significant in comparison to its in vivo elimination half-life. Under these conditions receptor occupancy can be maintained at levels leading to pharmacological responses even after a drug has been largely eliminated from systemic circulation. Under these circumstances we need to take the dynamics of binding into account when assessing selectivity. To illustrate this point let us assume that we have a kinase inhibitor that interacts with 3 
target kinases and that the rate constants $k_{1}\left(1 \times 10^{8} \mathrm{M}^{-1} \mathrm{~s}^{1}\right.$, i.e. 10 -fold below the diffusional limit), $k_{2}\left(1 \mathrm{~s}^{-1}\right)$, and $k_{3}\left(6 \times 10^{-3} \mathrm{~s}^{-1}\right)$ are identical in each case, and that only $k_{4}$ varies: $8.5 \times 10^{-3} \mathrm{~s}^{-1}$ (target 1), $2.8 \times 10^{-4} \mathrm{~s}^{-1}$ (target 2), and $1.26 \times 10^{-5} \mathrm{~s}^{-1}$ (target 3), i.e. typical values for type-I, type-II, and covalent reversible type-VI inhibitors. ${ }^{17,23}$ With these values we have residence times $T$ of 2 min (target 1 ), $1 \mathrm{~h}$ (target 2 ), and $22 \mathrm{~h}$ (target 3 ). If we further assume that the inhibitor was dosed orally once, reaching a maximum plasma concentration $\left(C_{\max }\right.$ ) of $10 \mu \mathrm{M}$ and being eliminated with a half-life of $3 \mathrm{~h}$, then we can calculate free plasma levels, as well as $f o_{t}$ with respect to the 3 targets, as a function of time as shown (Figure 3c). This analysis reveals that here selectivity is not driven by apparent affinity under equilibrium, but by residence time. Thus $>50 \%$ receptor occupancy is maintained for up to $12 \mathrm{~h}$ (target 1 ), $24 \mathrm{~h}$ (target 2), and $36 \mathrm{~h}$ (target 3). For targets 2 and 3 , significant receptor occupancy persists at a time when plasma levels would be undetectable using conventional bioanalysis methods ( $24 \mathrm{~h}, C_{f}=4.2 \times 10^{-10} \mathrm{M}$ ). Because the inhibitor dissociates from target 3 much more slowly than for targets 1 and 2 , it can be expected to be functionally selective for target kinase 3. Again the exact situation will depend on the magnitude and duration of receptor occupancy required to elicit physiological responses from the 3 targets, but it is clear that residence time is important in terms of selectivity and should be considered in the drug discovery process. ${ }^{24}$

\section{Target validity}

It is important to remember that the pioneer kinase inhibitor drug imatinib itself is not mono-selective for $B C R-A B L$, the oncogenic kinase uniquely present in chronic myelogenous leukaemia ( $C M L)$, but also inhibits a range of other kinases with high potency. Clearly the therapeutic effectiveness of imatinib in CML is not principally a result of drug selectivity, but the vital dependence of CML cells on BCR-ABL kinase activity. So for the narrow versus broad drug selectivity discussion to make any sense, we need to consider target validity.

If pharmacological modulation of a specific cellular component is sufficient to eradicate a diagnostically distinct cancer type, then surely an agent highly selective for that target should be preferable to a more promiscuous one on basic pharmacological grounds. If, on the other hand, the viability of a particular cancer type only appears susceptible to interference with two or more aberrant components, then multi-targeted agents would seem to be called for. Because we want to devise new cancer therapies that are inherently less toxic than conventional chemotherapies, the important thing is specificity for cancerous cells rather than inherent target selectivity.

If we accept the premise that at least for some cancer indications modulation of multiple targets is necessary to achieve the desired therapeutic effect, then the question that poses itself is whether it would be better to employ an appropriate cocktail of highly selective 
drugs or a single multi-targeted drug. ${ }^{25,26}$ Considering the complexity of human cancers, one would expect that the availability of a range of highly targeted agents would allow a more effective and modular approach to personalised therapy than multi-targeted agents. However, combination therapies are prone to dosing errors and drug-drug interactions. ${ }^{27}$ Furthermore, highly targeted drugs by definition will not find broad chemotherapeutic application but will only work in the (perhaps small) subset of cancers that possess the specific targeted molecular lesion, whereas multi-targeted drugs may find wider therapeutic use, i.e. they may be preferable commercial propositions for pharmaceutical companies.

As it turns out, the main clinical indication for imatinib, CML, is unusual since most cancers, especially non-haematological tumours, including e.g. common epithelial neoplasms of the lung, breast, colon, and prostate, display a much more complex transformed genotype than CML and may lack the critical vulnerability centred on a single cellular component characteristic of early-stage CML. In fact mouse models suggest that the $B C R-A B L$ translocation is the only genetic abnormality required to cause CML. ${ }^{28}$ It thus follows that imatinib probably represents an exception rather than a new paradigm in the area of kinase inhibitors. $^{29}$

However, target validation work in several areas suggests that there are probably many other cancers that may also possess therapeutic Achilles heels. Typically this situation arises as a result of oncogene addiction, i.e. the apparently paradoxical dependence of a tumour cell for sustained proliferation and survival on a single oncogenic pathway or protein, despite the presence of numerous genetic alterations. ${ }^{30} \mathrm{~A}$ number of such oncogene addictions have been identified and frequently the relevant oncogene products are kinases. ${ }^{31,32}$ Such kinases are attractive as cancer drug targets, and several are now being targeted with experimental drugs, e.g. BRAF in melanoma, HER2 in breast cancer, HER1 in non-small cell lung cancer (NSCLC), MET in gastric cancer, and ARK in colon cancer. However, unlike in the case of $\mathrm{CML}$, these addictions are not usually present in all cancers of a particular histological type, but the potentially responsive cancers need to be identified by examination of appropriate biomarkers.

\section{Drug resistance}

Although not themselves mutagenic like many conventional chemotherapy agents, kinase inhibitor drugs, just like most anticancer drugs, are nevertheless prone to both intrinsic and acquired resistance. The manifestation of drug resistance in at least a proportion of cases involves direct mutation of the target kinase in such a way that function is preserved, while inhibition by the drug is circumvented. Although it remains unclear how these mutations are caused, it is likely that genetic selection for insensitive target kinase mutants under the influence of the kinase inhibitor drug plays a role. This phenomenon was first 
observed with imatinib and is in part responsible for the current emphasis on MTKIs, which may not be vulnerable to the same extent to this selection pressure. ${ }^{33}$

In the case of the HER1 inhibitors gefitinib and erlotinib, which are used for the treatment of NSCLC with certain somatic mutations in the HER1 Tyr kinase or with amplified wildtype $H E R 1$, it has also been observed that all patients eventually develop resistance, either through a secondary HER mutation T790M in about half of all cases, by MET oncogene amplification, or via other mechanisms. ${ }^{34}$ The HER1 T790M mutation concerns the gatekeeper residue (Thr in most Tyr kinases), which is not required for the binding of the natural cofactor ATP, but which is almost invariably involved in the recognition of ATPantagonist kinase inhibitors. Resistance to HER2 inhibitors in breast cancer are also frequent and also develops through multiple mechanisms but unlike for HER1 inhibitors, e.g. lapatinib resistance due to the HER2 gatekeeper T798I mutation, appears to be less prevalent. ${ }^{35}$ Resistance mutations corresponding to those observed with HER Tyr kinase inhibitors are known to be important in the case of many other kinases and their inhibitors, as we shall see later.

It has long been known that cancer cells can develop drug resistance much more rapidly than would be predicted from the rates of conventional mutation, by differential utilisation of the genome, while normal cells in the same organism remain sensitive. There is now much evidence that not only point mutations, but also deletions, translocations, amplifications, epigenetic changes, and even altered microRNA levels, can generate cancer cell progeny tolerant to individual or multiple drugs. ${ }^{36}$ It is also now believed that clonal karyotype alterations are generated auto-catalytically by cancer-specific aneuploidy and that these specific chromosomal alterations cause drug resistance by changing the stoichiometry and integrity of multigenic transcriptomes. ${ }^{37}$ Even in the case of imatinib over half of all resistance cases are associated with clonal karyotype alterations rather than kinase mutations. $^{38}$

\section{Toxicology}

For the discussion of kinase inhibitor toxicology we need to return to the question of selectivity. Provided a hypothetical mono-selective kinase inhibitor anticancer drug does not give rise to toxic metabolites, one would expect such a compound to possess a clean toxicology profile. Obviously this will only be the case, however, if on-target effects in tissues other than the tumour can be tolerated. Again we can use the pioneer kinase inhibitor drug imatinib to illustrate this point. Although one of the targets of imatinib, $B C R-A B L$, is uniquely present in transformed granulocytes as a result of an oncogenic chromosomal translocation, the normal non-fused ABL kinase, which is widely expressed in the body, is of course also inhibited by imatinib. This kinase turns out to be especially important for cardiomyocyte function and survival, and $A B L$ inhibition has been identified as the root 
cause of the cardiotoxicity of imatinib. ${ }^{39}$ Similarly, hypo-phosphataemia is probably another on-target toxicity of imatinib, resulting from inhibition of PDGFR kinase activity in osteoclasts. ${ }^{40}$

Various other toxicities are target-related, such as the skin toxicity of HER inhibitors. ${ }^{41}$ Dermatological toxicities are also a feature of the multi-targeted kinase inhibitor sorafenib, currently approved for the treatment of metastatic clear-cell renal cell carcinoma and hepatocellular carcinoma, but it is not understood if this is target-related. ${ }^{42}$ Animal studies show that chronic pharmacologic inhibition of HER leads to cardiac dysfunction. ${ }^{43}$ Cardiac toxicity -drugs causing heart muscle valve damage or potentially fatal arrhythmias- has been implicated in $28 \%$ of drug withdrawals in the USA over the last 30 years. That cardiac and skin-related toxicities have been observed for many Tyr kinase inhibitors both in oncology and other indications suggests the possibility of a class effect. ${ }^{44}$ Another on-target toxicity is hyperglycaemia, which has often been reported as a side effect of agents targeting IGFIR, PI3K, AKT, and mTOR. All these kinases are important components of the insulin signalling pathway and their inhibition leads to altered hepatic glycogen metabolism and blockage of peripheral glucose uptake. It was shown recently, however, that drug-induced hyperglycaemia might be attenuated by decreasing liver glycogen storage using fasting, combined with a low-carbohydrate diet. ${ }^{45}$

\section{Approved and experimental kinase inhibitor drugs}

An overview of approved and experimental oncology kinase inhibitor drugs is given in Figure 4 and individual agents are discussed below by the kinases they act on. Details for compounds are summarised in Tables S2-S29.

BCR-ABL kinase. The $B C R-A B L$ oncogene is generated in CML by the so-called Philadelphia chromosome that results from a reciprocal translocation that juxtaposes the Abelson murine leukaemia viral oncogene homolog $1(A B L)$ gene with the breakpoint cluster region $(B C R)$ gene. ${ }^{46}$ Since its approval, the first $A B L$ kinase inhibitor imatinib has been used successfully in the control of progression from CML chronic phase to blast crisis. Whereas medial survival in CML was 3 to 6 years prior to the clinical use of imatinib, a 10-year survival rate of $68 \%$ has been reported for patients with CML who receive imatinib after failure of interferon. ${ }^{47}$

A $2^{\text {nd }}$ generation of $A B L$ inhibitors has now been approved: dasatinib and nilotinib, predominantly because many patients with CML undergo clinical relapse due to resistance to imatinib while these new agents are effective against most imatinib-resistant $B C R-A B L$ mutants (Figure 5). ${ }^{48}$ Neither imatinib nor the $2^{\text {nd }}$-generation $A B L$ inhibitors are selective, but also inhibit a range of other kinases, especially PDGFR and SCFR. In fact some of these agents have been used in the treatment of dermatofibroma sarcoma protuberans (DFSP) 
and gastrointestinal stromal tumours (GIST), in which disorders PDGFR and SCFR, respectively, are implicated. ${ }^{49}$

Bosutinib is a $3^{\text {rd }}$ generation $A B L$ inhibitor. Like the $2^{\text {nd }}$-generation inhibitors, it is also active against the majority of imatinib-resistant $B C R-A B L$ mutants, but it is a much more selective inhibitor, with only SRC kinases as significant additional targets. ${ }^{50}$ The other approved $3^{\text {rd }}$-generation inhibitor, ponatinib, on the other hand, is a multi-targeted kinase inhibitor, but it is the only approved $A B L$ inhibitor that has activity against the imatinibresistant T315I gatekeeper BCR-ABL mutant kinase (Figure 5). ${ }^{51}$ Another BCR-ABL inhibitor, radotinib (structurally closely related to imatinib), was approved in South Korea in 2012.

Although there are currently six approved $A B L$ inhibitors used successfully for the treatment of $C M L$, there are at least another two $A B L$ inhibitors at various stages of clinical development, including flumatinib (another compound closely related to imatinib) and $A B L$ 001, an allosteric (type IV) inhibitor that targets the myristoyl-binding site in the SH1 catalytic kinase domain (Table S2).

SRC kinases. A number of $A B L$ kinase inhibitors approved in $C M L$, such as dasatinib, nilotinib, bosutinib, and ponatinib also inhibit the closely related SRC kinases. SRC family kinases are cytosolic Tyr kinases that have a multitude of functions in cellular signal transduction, whose activated forms are aberrantly expressed in many solid tumours, especially metastatic cancers. ${ }^{52}$ This has led to clinical evaluation of such dual ABL and SRC agents, including saracatinib and AZD 0424 (closely related compounds that share the (6-chloro$2 H$-[1,3]dioxolo[4,5-b] pyridin-7-yl)aminoquinazolin-7-yl substructure), in advanced solid tumours such as NSCLC, as well as in lymphomas. ${ }^{53}$ Saracatinib is also currently being evaluated in Alzheimer's disease as an inhibitor of the SRC family kinase FYN, which is implicated in the pathogenesis of this disease. ${ }^{54}$ The most SRC-selective experimental kinase inhibitor drug (Table S3) appears to be a compound known as KX 01, which is a typeIII kinase inhibitor, i.e. a peptidomimetic of SRC kinase substrate peptides (Figure 6).

To date, therapeutic success in clinical trials of SRC kinase inhibitors has been limited and it has been postulated that a paradigm shift is required in clinical trial design for such agents in terms of patient selection, biomarker development, and rational design of combinatorial regimens with SRC inhibitors. ${ }^{55}$ However, recent insights suggest that highly selective SRC kinase inhibitors may possess significant advantages over dual ABL/SRC inhibitors, since tumour suppressor signalling through $A B L$ appears to occur at least in some cancers. ${ }^{56}$ Inhibitors that are highly selective for SRC over ABL have recently been reported, and the clinical development of such compounds may overcome this issue, as well as the cardiotoxicity associated with $\mathrm{ABL}$ inhibition. ${ }^{57}$ 
Epidermal growth factor receptor kinases. The EGFR Tyr kinase family consists of four transmembrane receptors, HER1-4, and signalling through this receptor family is important for many cell functions and for cell survival. At least HER1-3 are involved in tumourigenesis, and hetero-dimerisation of these receptors plays an important role in their function. ${ }^{58}$ Inhibitors of the EGFR Tyr kinase family have been studied mostly in breast cancers and NSCLCs driven by HER2 and HER1, respectively. As a consequence, activating mutations in e.g. EGFR (such as exon 19 in-frame deletions and L858R point mutations) serve not only as the rationale for the use of EGFR Tyr kinase inhibitors, but also as biomarkers for patient selection. ${ }^{59}$ Of the approved EGFR inhibitors, both gefitinib and erlotinib (Figure 7b) are HER1-specific and are used in NSCLCs, many of which overexpress HER 1.60

The trend in new EGFR Tyr kinase inhibitors is now towards pan-HER-targeted compounds, including the approved agent lapatinib (Figure 7d), which inhibits both HER1 and HER2 and is used for the treatment of advanced HER2-positive breast cancer. ${ }^{61}$ Icotinib, which is approved in China, is also an inhibitor of both HER1 and HER2. Although HER2 lacks an extracellular ligand-binding domain, it is nevertheless involved in hetero-dimerisation with other ligand-bound EGFR partners. Because EGFR signalling that affects cell proliferation and survival depends on both HER1 and HER2, inhibitors that block the catalytic activity of both have been sought, in the hope of enhanced clinical efficacy. However, clinical studies of pan-HER inhibitors have been difficult due to a narrow therapeutic margin with such agents, with dose-limiting on-target pan-HER toxicities such as diarrhoea and skin rash. ${ }^{62}$ Although the HER2-specific antibody drug trastuzumab has been used successfully in HER2-overexpressing breast cancer for some time, ${ }^{63}$ it is perhaps surprising that more progress has not been achieved to date with HER2-selective small-molecule kinase inhibitors. Highly HER2-selective inhibitors are in fact known (Figure 7c) ${ }^{64}$ but none seem to be under clinical evaluation at present.

Both acquired and innate drug resistance appear to play an important role in limiting the response rates to EGFR Tyr kinase inhibitor drugs. The comparatively unique presence of an unpaired Cys residue in the ATP-binding pocket of the EGFR Tyr kinases has allowed the development of irreversible ATP-site inhibitors. ${ }^{65}$ In preclinical studies it has been shown that cancers that have become resistant to gefitinib and erlotinib through the secondary EGFR T790M mutation (in ca. $60 \%$ of cases) remain sensitive to $2^{\text {nd }}$-generation type-VI inhibitors. ${ }^{66}$ This, and the observation that such inhibitors may be less likely to induce drug-resistant kinase mutants, might be due to the fact that covalent inhibitors can still block a mutant kinase that has reduced affinity for non-covalent inhibitors, because with covalent binders inhibition is rate- rather than affinity-driven. ${ }^{67}$ Two such agents that can overcome T790M mutation, afatinib (Figure 7f) and osimertinib (Figure 7g), were re- 
cently approved in metastatic NSCLC. Afatinib is a pan-HER inhibitor that blocks the catalytic activity of HER1, HER2, and HER4, as well as phosphorylation of the EGFR-transsignalling partner HER3, ${ }^{68}$ which is itself kinase-inactive. ${ }^{69}$ Osimertinib, on the other hand, is selective for T790M-mutant HER1, as well as L858R and exon-19 deletion mutant forms of HER1. Because osimertinib spares wild-type HER1, it may have reduced toxicity compared to afatinib and other EGFR inhibitors used in NSCLC. ${ }^{70,71}$

Apart from the six small-molecule EGFR Tyr kinase inhibitors already approved, no fewer than four type-VI inhibitors are currently in late-stage clinical development, including dacomatinib and neratinib (pan-HER and T790M HER1), as well as ASP 8273 and rociletinib (T790M HER1-selective). At least another 16 agents, with varying selectivity profiles, are undergoing clinical development (Table S4).

CSF1/PDGF receptor subfamily kinases (Table S5). The CSF1/PDGF Tyr kinase subfamily contains a number of closely related kinases that are relevant to cancer, including CSF1R, VEGFR/FLT, PDGFR, and SCFR (c-Kit). Vascular endothelial growth factors (VEGFs) act on three main receptor Tyr kinase receptors, i.e. VEGFR1, VEGFR2, and VEGFR3, whereas platelet-derived growth factors (PDGFs) act on the PDGFRa and PDGFR $\beta$ receptors, which contain similar Tyr kinase domains. Both VEGFRs and PDGFRs activate cellular proliferation and survival signalling pathways, such as the RAF-MAP-MEK-ERK and the PI3K-AKT pathways. These receptors are implicated in tumour angiogenesis, metastasis, and progression. ${ }^{72,73}$

All currently approved (and many of the late-stage development) compounds that inhibit CSF1/PDGF Tyr kinases are MTKIs and contain some of the original kinase inhibitor core templates, $^{74}$ e.g. sunitinib and nintedanib are 3-methylidene-2-oxo- $1 H$-indoles, vandetanib is a 4 -(anilino)quinazoline, and pazopanib is a bis(arylamino)pyrimidine. These agents are variously used in the treatment of advanced renal cell carcinoma (sunitinib, axitinib, pazopanib), gastrointestinal stromal tumours (sunitinib), advanced or metastatic pancreatic neuroendocrine tumours (sunitinib), advanced soft tissue sarcoma (pazopanib), late-stage medullary thyroid cancer (MTC; vandetanib), and progressive, radio-iodine-refractory differentiated thyroid cancer (lenvatinib). Nintedanib is another approved MTKI with activity against CSF1/PDGF kinases. It received its first global approval not in oncology but for the treatment of IPF. The activity in this indication may be linked to the ability of nintedanib, amongst other kinases, potently to inhibit FGFR Tyr kinases, as aberrant FGFRs appear to contribute to the pathogenesis of IPF (see below). ${ }^{75}$ Nintedanib is also now used for the treatment of advanced, metastatic NSCLC in combination with docetaxel. Numerous additional VEGFR inhibitors with varying selectivity profiles are now being developed. E.g. fruquitinib and cediranib are selective pan-VEGFR/PDGFR inhibitors. 
Although there is abundant evidence that angiogenesis inhibitors can suppress tumour development in preclinical models, recent findings show that pharmacological as well as genetic VEGFR suppression can in fact alter tumour development by increasing invasion and metastasis. ${ }^{76,77}$ In the clinic, the response to VEGFR inhibitors is often limited and followed by disease progression that is at least in part due to tumour escape as a result of VEGFR inhibition. For these reasons research and clinical trialling of drug combination strategies that may prevent such escape mechanisms are on-going. ${ }^{78}$

Closely related to the VEGFR Tyr kinases is FLT3, which is commonly overexpressed in most B-lineage leukaemias (acute lymphocytic leukaemia, ALL; acute myelogenous leukaemia, AML), in subsets of T-cell ALL, and CML in blast crisis. Furthermore, FLT3 activating mutations are present in about a third of $\mathrm{AML}$ patients. ${ }^{79} \mathrm{~A}$ number of multi-targeted CSF1/PDGF TK inhibitors with activity against FLT3 are being developed, e.g. midostaurin, AKN 028, and PLX 7486. Quizartinib, a benzo[d]imidazo[2,1-b]thiazol-2-yl)phenyl)urea compound, is an example of a late-stage selective type-II ${ }^{80}$ FLT3 inhibitor being trialled in the treatment of ALL. ${ }^{81}$ Unusually for a type-I TK inhibitor, crenolanib has high selectivity for FLT3, SCFR, and PDGFR kinases, including resistance-conferring kinase domain mutant forms. ${ }^{82}$ This finding supports the emerging notion that type-II inhibitors may not necessarily be intrinsically more selective than type-I inhibitors, as has been thought in the past. $^{83}$

Among the CSF1/PDGF Tyr kinases is SCFR. Oncogenic signalling from mutant KIT is especially common in GIST $^{84}$ and currently the standard therapy for unresectable or metastatic GISTs is first-line imatinib, second-line sunitinib, and third-line regorafenib. ${ }^{85}$ All three of these agents are MTKIs that were originally developed for alternative indications, but more selective SCFR inhibitors are also now being developed, e.g. crenolanib and PLX 9486. The D816V KIT activating mutation occurs in > 90\% of patients with systemic mastocytosis and whereas imatinib is not efficacious in this indication, dasatinib has been shown to be active. ${ }^{86}$

CSF1R is a cell-surface receptor for both macrophage colony-stimulating factor-1 (CSF1) and interleukin-34. It plays an important role as a regulator of tissue and tumour-associated macrophages. ${ }^{87}$ Because CSF1R activation promotes tumour progression through suppression of the anti-tumour immune response, as well as promotion of angiogenesis and metastasis, CSF1R inhibitors may be particularly useful in many cancer indications. In fact many MTKIs from the CSF1/PDGF class also inhibit CSF1R and this activity may contribute to their efficacy. However, selective CSF1R inhibitors are also now under development, e.g. pexidartinib and ARRY 382 (undisclosed structure).

Fibroblast growth factor receptor kinases. The four isoforms (FGFR1-4) of this receptor Tyr kinase family are structurally and functionally related to the HER family and bind 
diverse FGF ligands. FGFR signalling appears to be implicated in the pathogenesis of several cancers and FGFR aberrations are frequent (7.1\% of cancers), especially in urothelial, breast, endometrial, and lung cancers. Apart from finding potential therapeutic application against tumours with FGFR genetic alterations, FGFR inhibitors may also be useful to overcome resistance against HER inhibitors, as alternative signalling through the FGFR pathway apparently represents an important escape mechanism when HER signalling is suppressed. Conversely, FGFR kinase inhibitors may be able to potentiate HER inhibition. ${ }^{8}$

Many of the approved MTKIs in the CSF1/PDGF class also inhibit FGFR kinases but more selective FGFR kinase inhibitors are also now under development. AZD 4547, infigratinib, and erdafitinib (Figure $8 \mathrm{a}$ ) are potent and selective pan-FGFR inhibitors currently undergoing phase-II trials in a range of cancers. Another 7 FGFR kinase inhibitors (structures not disclosed) are currently in phase-I trials (Table S6). At least two of these are irreversible inhibitors (PRN 1371; TAS 120, refer Figure 8e), whereas another is an isoform-selective FGFR4 inhibitor (FGF 401).

One of the mechanisms of acquired resistance to FGFR inhibition is gatekeeper mutation. Thus V561M mutation in FGFR1 confers significant resistance to e.g. lucitanib (Figure 8c) but not dovitinib (Figure 8d). Interestingly AZD 4547 retains affinity for the V561M mutant FGFR1 by adopting an alternative binding mode in which the flexible dimethoxyphenethyl group -which is in contact with the wild-type V561 gatekeeper- is able to bend away from the larger M561 gatekeeper in the mutant kinase. ${ }^{89}$

Hepatocyte growth factor receptor kinase (HGFR or MET). The MET Tyr kinase is a cell-surface receptor for hepatocyte growth factor, whose downstream signalling pathways are important for invasive growth in terms of motility, proliferation, and protection from apoptosis. These processes are important in embryonic development and tissue morphogenesis but often become reactivated in cancer, especially in tumour metastasis. Constitutive MET activation is a feature of many cancers, ${ }^{32}$ including NSCL carcinomas with acquired resistance to EGFR inhibitors. ${ }^{90}$ MET amplification can be a primary or secondary transformation event and e.g. in some patients with colorectal carcinomas, MET amplification has been observed not in primary tumours, but in liver metastases. ${ }^{91}$

The first MET Tyr kinase inhibitor to be approved for the treatment of patients with progressive metastatic MTC is cabozantinib, a MTKI with MET and VEGFR2 as its main targets. ${ }^{92}$ A range of functionally different (Figure 9) selective and MTKI MET inhibitors are currently under clinical evaluation in a number of cancers, including NSCLC resistant to EGFR inhibitors. ${ }^{93}$ The most advanced of these is tivantinib, a highly selective MET inhibitor. Second-generation inhibitors that are active against mutant forms of MET, such as tepotinib, are also being developed (Table S7). 
Anaplastic lymphoma kinase (Table S8). ALK is a receptor Tyr kinase in the insulin receptor family and $A L K$ rearrangement is second only to $H E R$ mutation as an oncogene addiction in NSCLC. Modification of $A L K$ is present in only a small proportion of NSCLC patients but specific targeting of this population for treatment with ALK inhibitors is particularly effective. ${ }^{94}$ The first ALK inhibitor approval in NSCLC was crizotinib, a MTKI originally discovered and developed as a MET inhibitor and now prescribed successfully as firstor second-line therapy in ALK-positive NSCLC. ALK rearrangements also occur in $>50 \%$ of anaplastic large cell lymphomas (ALCLs) and crizotinib has been shown to have clinical activity in advanced chemoresistant ALCL. ${ }^{95}$

The development of acquired resistance is the main reason why patients with ALK-positive NSCLC eventually relapse on crizotinib. Secondary resistance mutations in the ATP-binding site have been identified ${ }^{96}$ and one of the main drivers for the development of secondgeneration ALK inhibitors has been to overcome crizotinib resistance. ${ }^{97}$ Ceritinib was approved later and is a more potent ALK inhibitor that overcomes the most common secondary mutation to crizotinib (gatekeeper L1196M), as does the recently approved selective ALK inhibitor alectinib.

These second-generation ALK inhibitors also show activity against brain metastases, which is important, as almost half of ALK-positive NSCLC patients on crizotinib exhibit such metastases, even when systemic disease is under control. ${ }^{98}$ Three more third-generation ALK inhibitors are currently undergoing clinical evaluation: brigatinib, belizatinib, and lorlatinib. At least the latter compound was designed specifically to have high CNS penetrance (Figure 10). ${ }^{99}$

IGF1R and IR. The insulin-like growth factor 1 (IGF1) signalling pathway regulates many aspects of the proliferation, differentiation, and growth of normal cells. However, its receptor, IGF1R, as well as its ligands and adaptor proteins, are highly expressed in many malignancies. Furthermore, the IGF1 pathway is also implicated in carcinogenesis and cancer risk. IGF1R activates the RAS-RAF-MAPK and PI3K-AKT-mTOR pathways, both of which are associated with cancer cell proliferation and survival. For these reasons IGF1R inhibitors have been developed, especially in NSCLC. ${ }^{100}$ The first generation of IGF1R inhibitors that have been tested in clinical trials are humanised monoclonal antibodies that block ligand binding to IGF1R. Although early results were promising, later studies using combinations with chemotherapy or EGFR inhibitors in NSCLC have been disappointing. ${ }^{101,102}$ Currently the only clinical small-molecule IGF1R inhibitor targeted at the Tyr kinase activity of this receptor is the phenylquinoline compound linsitinib, a dual IGF1R and insulin receptor (IR) inhibitor (Table S29).

Tropomyosin receptor kinase (Table S9). TRK was originally identified as an oncogene product that arises as a result of mutations and rearrangements of the NTRK genes and has been detected in various tumours, especially in the colon, the lung, and the thyroid 
gland. ${ }^{103}$ The three isoforms of wild-type TRK are expressed predominantly in neurons, however, where their activation by neurotrophins plays important roles in cell development, maintenance, and function. It is now clear that TRKs also regulate processes in nonneuronal cells. Furthermore, activation of cell survival pathways through TRKs contributes to tumourigenesis and resistance to cytotoxic chemotherapy not only in neuroblastoma, but also in many other cancers. ${ }^{104}$ The TRKs belong to the same insulin receptor Tyr kinase subfamily as ALK and ROS, and entrectinib, a pan-TRK inhibitor with additional potent ALK and ROS inhibitory potency, is currently undergoing phase-II trials in colorectal carcinoma and NSCLC. ${ }^{105}$ Another agent at a similar stage of development is LOXO 101, a selective pan-TRK inhibitor. Entrectinib and LOXO 101 are structurally related, the former contains an indazole, the latter a pyrazolo[1,5-a]pyrimidine core, both compounds containing a meta-difluorophenyl substituent.

AXL belongs to the TYRO3, AXL, and MERTK (TAM) family of receptor Tyr kinases and although it does not appear to be a strong oncogenic driver, it is overexpressed in many tumours and overexpression correlates with poor prognosis. Aberrant TAM signalling in cancer suggests that $A X L$ inhibitors may confer anti-tumour immunity and tumour cell survival, as well as enhance chemosensitivity and suppress metastases. ${ }^{106}$ It is somewhat difficult to decide which development compounds should be designated as AXL inhibitors, since several MTKIs, including approved agents such as cabozantinib, bosutinib, crizotinib, and sunitinib, are also potent $A X L$ inhibitors. ${ }^{107}$ One of the most potent $A X L$ inhibitors (sub$\mathrm{nM} \mathrm{IC}_{50}$ ) is the pyrazinecarboxamide gilteritinib, which also potently inhibits FLT3 and ALK. ${ }^{108}$ The first selective (at least in terms of cell-based kinase inhibition activity) AXL inhibitor to enter the clinic is BGB 324, a substituted triazole-3,5-diamine that contains two instances of the unusual tetrahydrobenzannulene substructure (Table S10).

RET (REarranged during Transfection) is a receptor Tyr kinase whose activity supports cell survival and proliferation, as well as cell migration, differentiation, and chemotaxis. Activating mutations in RET (C634W and M918T) and a number of different gene translocations leading to oncogenic fusions involving RET occur in various cancers, particularly in MTC and NSCLC. ${ }^{109}$ RET is closely related to other receptor Tyr kinases, especially VEGFR, and many MTKIs possess RET-inhibitory activity (refer Tables S5, S7, S9). As we have seen, vandetanib, lenvatinib, and cabozantinib are approved for use in MTC and the activity of these agents in thyroid cancers -as well as in some lung cancers- is probably due to a large extent because they inhibit RET. ${ }^{110-113}$ However, the efficacy of MTKIs, especially those that also block VEGFR activity, is limited by toxicities ${ }^{114}$ and selective RET inhibitors are highly desirable for the treatment of cancers that are driven by RET activation. Although research aimed at the discovery of such agents is under way, ${ }^{115,116}$ there do not appear to be any RET-specific kinase inhibitors under clinical evaluation at present. 
Transforming growth factor- $\boldsymbol{\beta}$ receptor. A range of cellular responses, including proliferation, differentiation, motility, and survival, are elicited upon activation by the TGF- $\beta$ cytokines of the three TGFR isoforms through receptor hetero-dimerisation. However, in normal cells TGF- $\beta$ actually functions as a tumour suppressor but TGF- $\beta$ signalling is highjacked by cells during tumour development and is particularly important during the epithelial-mesenchymal transition. ${ }^{117}$ TGFRs contain Ser/Thr kinase domains and signal transduction occurs through TGFR heterodimer-mediated phosphorylation of cellular proteins such as SMADs, which activate the TGF- $\beta$ signalling pathway. ${ }^{118}$ Drugs, including antisense oligonucleotides, antibodies, and small molecules targeting this pathway have been under development in both neoplastic and non-neoplastic indications, especially in fibrosis. ${ }^{119}$ Two TGFR1 kinase inhibitors are currently being developed in oncology, galunisertib and TEW 7197, both of which contain the 2-methyl-6-(4H,5H,6H-pyrrolo[1,2-b]pyrazol-2-yl)pyridine substructure (Table S11).

Janus kinases. The JAK family includes the 3 cytoplasmic Tyr kinases JAK1, JAK2, and JAK3, as well as the non-receptor Tyr kinase TYK2. These kinases transmit signalling from a number of cytokines and growth factors important in haematopoiesis and cellular immune functions. Whereas JAK3, which is critical to signal transduction via common $y$-chains of interleukin receptors, is pursued primarily as a drug target in inflammation, especially JAK2 is also an oncology drug target. The interest in JAK2 inhibitors stems from the discovery that many myeloproliferative neoplasms display an activating mutation (V617F) in the JAK2 gene. ${ }^{120}$

Ruxolitinib, a JAK1/2-selective compound, ${ }^{121}$ is the first JAK inhibitor in oncology, approved for the treatment of myelofibrosis. A recent survey of the clinical use of this agent concluded that ruxolitinib, currently the only approved therapy for myelofibrosis, is associated with a survival benefit and has changed the treatment landscape for this disease. ${ }^{122} \mathrm{Nev}$ ertheless, clinical responses have been attenuated and the original hope of curative effects with JAK2 inhibition similar to those seen with imatinib in $\mathrm{CML}$, has not been fulfilled. It has recently been suggested that this may be due to an intrinsic resistance to JAK2 inhibition in myelofibrosis. ${ }^{123}$

A number of other (structurally diverse) compounds (Table S12), including pacritinib (JAK2/FLT3-selective), momelotinib (JAK1-3-selective), are also currently undergoing clinical development in myeloproliferative neoplasms. ${ }^{124}$ It is not as yet clear how these agents will be differentiated from ruxolitinib and if they will have to be compared to ruxolitinib in randomised studies for the purposes of approval. ${ }^{122}$

Bruton tyrosine kinase. BTK is a non-receptor kinase in the B-cell antigen receptor (BCR) signalling cascade that mediates B-cell activation, proliferation, migration, and survival. In B-cell non-Hodgkin's lymphomas (NHL) constitutive BCR activation through BTK supports cell proliferation and survival, rendering BTK a relevant pharmacological target in NHL. 
This is especially attractive in terms of specificity, since the phenotype of loss-of-function BTK, as occurs in the human disease X-linked agammaglobulinaemia, is restricted to Bcells. ${ }^{125}$

The first potent and selective type-VI inhibitors of BTK were reported in 2007 and it took only 6 years before the first inhibitor of this type, ibrutinib, obtained initial global regulatory approval in the treatment of mantle cell lymphoma. The first BTK inhibitors of any kind were actually reported already in 1999: terreic acid (a quinone epoxide) ${ }^{126}$ and leflunomide metabolite analogues (2-cyano-3-hydroxybut-2-enamides). ${ }^{127}$ Since these compounds possess highly electrophilic functions, it is likely that they are also type-VI inhibitors that react covalently with the thiol of $\mathrm{C} 481$, an unpaired Cys residue in the ATP-binding site of BTK (corresponding to C773 in HER kinases), although this was not apparently realised at the time of their discovery. Interestingly, terreic acid was originally studied in in the context of mast cell activation and it was realised that BTK inhibitors may find therapeutic application in immunology and inflammation, whereas leflunomide metabolite analogues were reported as anti-leukaemic drug leads. Ibrutinib, too, was discovered in a rheumatoid arthritis drug discovery programme, ${ }^{128}$ and was later repositioned in B-cell malignancies. ${ }^{129}$ Apart from ibrutinib (a pyrazolo[3,4-d]pyrimidine) there are currently five additional BTK inhibitors under clinical development in cancer (Table S13). Of these the chemical structures of two are in the public domain and both are structurally closely related to ibutinib. Acalabrutinib is an imidazo[1,5-a]pyrazine derivative with a reactive but-2-enoyl function, whereas GS 4059 is a purin-8-one derivative with -like ibrutinib- an acryloyl electrophile.

Focal adhesion kinase. FAK1 is a cytoplasmic non-receptor Tyr kinase that transmits cell motility, survival, and proliferation signals from integrin-mediated cell extracellular matrix attachment and growth factor receptors. Because metastatic propensity and poor clinical outcomes of many cancers are correlated with enhanced FAK1 expression and activity, FAK1 is a promising target for cancer therapy. ${ }^{130}$

A number of FAK1 inhibitors have been under clinical investigation in the past, ${ }^{131}$ but currently only three remain active (Table S14). GSK 2256098 and VS 4718 are selective FAK1 inhibitors, whereas defactinib is a dual FAK1 and FAK2 inhibitor. Such dual selectivity is believed to be potentially beneficial due to functional overlap and redundancy of these two closely related kinases. ${ }^{132}$ All three compounds are structurally related ATP-competitive (presumably type-I) inhibitors, but allosteric FAK1 inhibitors have also been reported, ${ }^{133}$ although these have not yet reached clinical evaluation.

Spleen tyrosine kinase. SYK is a cytoplasmic non-receptor Tyr kinase expressed predominantly in haematopoietic cells. Phosphorylation of cytoplasmic immunoreceptor tyrosine-based activation motifs (ITAMs) leads to recruitment of SYK, which propagates signalling by activation of downstream pathways, including PI3K, MAPK, and BTK. ${ }^{134}$ Because 
SYK mediates cellular responses to antigens and antigen-immunoglobulin complexes it is especially relevant as a drug target to suppress the consequences of acute and chronic inflammation. ${ }^{134}$ However, SYK, together with BTK, is also involved in dysregulation of BCR signalling in haematological malignancies such as diffuse large $B$-cell lymphoma ( $D L B C L)$, follicular lymphoma, and chronic lymphocytic leukaemia (CLL). ${ }^{135}$

A number of SYK inhibitors have been evaluated clinically, mainly for the treatment of immune disorders, but were found to exhibit dose-limiting toxicities attributed predominantly to lack of sufficient selectivity for SYK. ${ }^{136}$ The first of these was fostamatinib (a methylene phosphate oral prodrug of a poorly soluble dianilinopyrimidine compound), which failed in phase-III trials in rheumatoid arthritis and has also been trialled in B- and T-cell lymphoma, but apparently has not progressed. ${ }^{137}$ Second-generation SYK inhibitors with improved kinome selectivity profiles have now been developed and entered the clinic (Table S15). The most advanced of these is entospletinib, a highly SYK-selective oral disubstituted imidazo[1,2-a]pyrazine compound, currently in phase-II studies in both inflammation and cancer (haematological malignancies). Cerdulatinib is a dual JAK and SYK inhibitor (Table S12) that has activity in autoimmunity and B-cell cancers like other SYK inhibitors. ${ }^{138}$

PI3K pathway kinases (Table S16). The PI3K pathway regulates protein synthesis, cell growth, and cell proliferation in response to nutrient availability and mitogenic growth factors. Activation of this pathway has long been associated with malignant transformation and anti-apoptotic signalling. ${ }^{139}$ Amplification or mutation of many receptor Tyr kinases, mutation of downstream signalling components, including PI3K itself, loss or mutation of the PTEN tumour suppressor protein, as well as mutation and amplification of $A K T$, can lead to pro-survival signalling through the PI3K-AKT-mTOR-eIF4E pathway in cancer cells. At least four kinases in this pathway, PI3K itself (i.e. the class-I PI3K isoforms $a-\delta$ ), AKT, its activating kinase PDK1, and mTOR, are being actively pursued as oncology drug targets. The first generation mTOR inhibitors are derived from the macrolide rapamycin (sirolimus), a natural product from a fungus indigenous to the Easter Island (Rapa Nui). Studies based on the observation that rapamycin inhibited the proliferation of T-cells led to the discovery of the mTOR (mechanistic or mammalian target of rapamycin) genes and the development of sirolimus as a targeted drug. Although the anticancer activities of rapamycin had been known for a long time, sirolimus was first developed as an immunosuppressive agent. However, a rapalogue (rapamycin analogue), temsirolimus, was approved for the use in advanced renal cell carcinoma (RCC). Objective responses of kidney cancer patients to rapalogues had been observed already in phase-I studies ${ }^{140}$ and the demonstration that loss of the von Hippel-Lindau tumour suppressor gene (VHL) sensitises kidney cancer cells to mTOR inhibitors subsequently enabled biomarker-driven development of temsirolimus in RCC. ${ }^{141}$ Another rapalogue, everolimus, was approved later for the same indication . 
mTOR participates in at least two functionally distinct multiprotein complexes termed mTORC1/RAPTOR and mTORC2/RICTOR. Rapalogues, which block mTOR activity indirectly in complex with the immunophilin receptor known as FK506-binding protein-12 (FKBP12), specifically inhibit mTORC1. ${ }^{142}$ It was reported recently that the rapalogue everolimus can also act as a MET inhibitor (refer section on hepatocyte growth factor receptor kinase), since MET -like mTOR- also requires FKBP12 binding for full activity. ${ }^{143}$ Because of the presence of mTORC1-dependent negative feedback loops via the mTOR phosphorylation substrate ribosomal protein S6 kinase $\beta$-1 (S6K1) leading to upregulation of PI3K and RAS activities, inhibition of mTORC1 can lead to activation of AKT- and MAPK-dependent survival pathways. ${ }^{144}$ For this reason, selective ATP-antagonist mTOR inhibitors, which block both mTOR complexes, were developed and are also now entering clinical trials. Clinical compounds belonging to this group include vistusertib, sapanisertib, and CC 223.

Unsurprisingly considering the similarity of mTOR and PI3K, the majority of ATP-antagonist mTOR inhibitors turned out to inhibit PI3K as well, or they were designed to possess dual specificity. Again several compounds of this type are being developed, e.g. the dual panclass I PI3K and mTOR kinase inhibitors voxtalisib, LY 3023414, PQR 309, gedatolisib, and VS 5584.

Inhibitors that do not modulate mTOR at all but inhibit various combinations of class-I PI3K isoforms are also now being pursued. This appears to be a valid approach as all four functionally non-redundant isoforms can generate the signalling molecule phosphatidylinositol3,4,5-trisphosphate ( $\left.\mathrm{PIP}_{3}\right)$, which recruits AKT to the cell membrane, and because all are oncogenic in model systems. ${ }^{145}$ Furthermore, all four isoforms are frequently genetically altered in a variety of cancers. ${ }^{146}$ At present it remains unclear what PI3K/mTOR inhibition selectivity (Figure 11) will be therapeutically optimal. It is likely that the preferred class-I PI3K target will depend on the cellular context. ${ }^{147}$ The main driver for seeking isoformselective PI3K inhibitors is probably because broad selectivity may limit the therapeutic margin of an mTOR/PI3K inhibitor, due to the important role of the PI3K pathways in normal cells. Currently there are several pan-class-I PI3K inhibitors undergoing clinical trials, including buparlisip, copanlisip, pictilisib, ZSTK 474, and SF 1126, the latter being a vascularly targeted prodrug.

That PI3Kס is only expressed in haematopoietic cells provides the therapeutic rationale for the use of the PI3Kס-selective compounds in haematological cancers ${ }^{148}$ and this concept has now led to the first approval of a PI3Kס-selective inhibitor, idelalisib as monotherapy for follicular and small lymphocytic lymphoma. Another compound, duvelisib, with a similar selectivity profile (10- to 40-fold selectivity over PI3KY and higher over other PI3Ks) is in late-stage development. Other clinical compounds with more or less pronounced PI3Kס selectivity include AMG 319, HMPL 689, INCB 050465, and CDZ 173. A recent study showed that PI3Kס (and PI3KY) is important for T-cell-mediated immune tolerance to cancer and 
PI3K $\delta$ inhibitors may therefore find therapeutic applications not just in blood cancers but perhaps much more widely. ${ }^{149}$

Apart from PI3Kס-selective inhibitors, a growing number of experimental PI3K inhibitor drugs with a range of PI3K isoform selectivity profiles are being investigated. The interest in PI3Ka-selective agents stems predominantly from the observation that the gene encoding this isoform is mutated particularly frequently in certain cancers (e.g. HER2- and KRASdriven tumours). ${ }^{150}$ The most advanced PI3Ka-selective compounds are alpelisib and talelisib; others include MLN 1117 and AZD 8835. Because PI3Ka specifically regulates glucose haemostasis, this must be carefully managed with PI3Ka-selective inhibitors in the clinical setting. ${ }^{151}$ Dependence on PI3K $\beta$ has been observed in certain PTEN-deficient tumours and the first PI3K $\beta$-selective agent, GSK 2636771 is now being trialled in this setting. Other PI3K $\beta$-selective clinical compounds include AZD 8186 and KA 2237.

Because of the importance of the PI3K pathway to cancer cell survival, it was originally thought that PI3K inhibitors might have anticancer efficacy in monotherapy, but with the clinical experience amassed so far with over 20 different PI3K/mTOR inhibitors, it is increasingly becoming clear that this is unlikely to be the case. Like most kinase inhibitors, PI3K inhibitors will probably work best in combination with other anticancer drugs. ${ }^{152}$

AKT (also known as PKB) is a Ser/Thr kinase that acts downstream of PI3K and is activated by recruitment to the cell membrane by interaction with PIP $_{3}$ through its $\mathrm{PH}$ (pleckstrin homology) domain. Once activated, AKT mediates downstream responses by phosphorylation of multiple proteins. The lipid phosphatase PTEN, which is frequently inactivated in human tumours, negatively regulates AKT by dephosphorylation of $\mathrm{PIP}_{3}$, whereas AKT is amplified or overexpressed in many tumours. ${ }^{153}$

Until recently the clinically most advanced AKT inhibitor was the alkylphospholipid perifosine. It is not a direct kinase inhibitor but interferes with membrane localisation of AKT. ${ }^{154}$ Phase-II clinical trials of perifosine as a single agent in several tumour types failed to show objective responses. ${ }^{153}$ Although promising activity in patients with RCC, who have failed prior Tyr kinase inhibitor therapy, was reported, ${ }^{155}$ perifosine does not appear to be under development any longer. Several type-I pan-AKT inhibitors, including AZD 5363, ipatasertib (Figure 12a,c), LY 2780301, and afuresertib are now in phase-II clinical studies and several more compounds are in early stage trials (Table S17), including the allosteric (type-III) AKT inhibitor MK 2206 (Figure 12a). This compound binds to an AKT site formed only in the presence of the $\mathrm{PH}$ domain and binding of the inhibitor promotes the formation of an inactive kinase conformation. ${ }^{156}$ MK 2206 is similar to compound VIII of ref. ${ }^{157}$, whose experimental binding mode is known (Figure 12b,c). ARQ 092 and BAY 1125976 are also allosteric AKT inhibitors. The structure of the latter compound has not yet been disclosed but the compound series that encompasses ARQ 092 is structurally related to compound VIII and MK 2206. ${ }^{158}$ 
PDK1 plays an important role in the PI3K-AKT pathway. It activates AKT, as well as a number of other related AGC kinases, thus controlling several signalling pathways important in proliferation, apoptosis, and angiogenesis. Like PI3K, PDK1 is upregulated in more than half of all tumours due to constitutive growth factor receptor activation and PTEN mutations. As expected, transgenic mice expressing low levels of PDK1 are protected from tumourigenesis that normally results from loss of PTEN. The search for potent and selective PDK1 inhibitors has been pursued for some time but as with many other kinases, achieving selectivity in inhibitors has proven challenging. ${ }^{159}$ Nevertheless, the first PDK1 inhibitor, AR 12, recently entered clinical trials (Table S29). This compound was designed from celecoxib, a cyclooxygenase-2 ( $\mathrm{COX} 2$ ) inhibitor, following the assignation of tumourpreventive properties of celecoxib to PDK inhibition. However, the activity and selectivity with which AR 12 inhibits PDK1 is actually uncertain. ${ }^{159}$

Protein kinase C. PKC overexpression has been linked to several types of cancer, with the PKC $\beta$ isoform believed to be involved in VEGF-induced tumour development and angiogenesis and in the apoptosis-regulating PI3K-AKT pathway. The macrocyclic bisindolylmaleimide enzastaurin selectively inhibits PKC $\beta$ at low concentrations, and also inhibits other PKC isozymes at higher concentrations that are reached or surpassed in clinical trials. ${ }^{160}$ Anti-tumour activity of enzastaurin has been primarily attributed to the inhibition of AKT and its downstream targets, but its precise mechanism of action is not well understood. ${ }^{161}$ Both PKC and AKT are activated by PDK, and several PKC isozymes can also directly phosphorylate AKT at Ser ${ }^{473}$, which is essential for AKT activity. It was recently reported that a phase-III clinical trial of enzastaurin in monotherapy in diffuse large B-cell lymphoma failed to show a statistically significant increase compared to placebo in diseasefree survival in patients at high risk of relapse following rituximab-based chemotherapy, ${ }^{162}$ and apparently development of this compound has been discontinued.

Enzastaurin is not the first staurosporin derivative to undergo clinical development, e.g. UCN-01 (7-hydroxystaurosporin) and midostaurin, which also inhibit PKC but have been developed as MTKIs (main targets are CHK1 and PDK1 for UCN-01, and FLT3 and other CSF-1/PDGF receptor subfamily kinases for midostaurin). ${ }^{163,164}$ Other staurosporine derivatives are being developed in non-oncology indications. ${ }^{165}$ The only PKC inhibitor that remains under clinical study appears to be LXS 196, an oral compound whose structure or properties have not yet been disclosed (Table S29).

Choline kinase-a. CK is a lipid kinase that phosphorylates free choline to give phosphocholine, enabling the formation of phosphatidylcholine, the main phospholipid in mammalian cell membranes, through the Kennedy pathway. Many tumours display altered lipid metabolism during development and as a consequence of chemotherapy CK is up-regulated in many tumours and is associated with various malignant phenotypes. ${ }^{166}$ Further- 
more, CK was recently reported as an androgen receptor chaperone and potentially valuable drug target in prostate cancer. ${ }^{167,168}$ TCD 717 is the first CK inhibitor to have recently entered clinical trials in solid tumours (Table S29). It inhibits CK by virtue of competing with the substrate choline; its structure has not been disclosed but is related to hemicholinium-3 ((2S,2'S)-2,2'-biphenyl-4,4'-diylbis(2-hydroxy-4,4-dimethylmorpholin-4-ium), a choline re-uptake inhibitor. ${ }^{169}$

Casein kinase II is a constitutively active hetero-tetrameric Ser/Thr kinase that sustains many cellular signalling pathways. Although CK II function is not apparently altered in cancer cells by mutation, it is nevertheless overexpressed in many different cancers. ${ }^{170}$ Tumour cells often exhibit CK II-driven non-oncogene addiction ${ }^{171}$ and it has been shown that ablation of CK II through antisense oligonucleotides has profound anti-proliferative and pro-apoptotic effects in a rodent xenograft model. ${ }^{172} \mathrm{~A}$ number of small-molecule CK II inhibitors with anticancer activity have been reported and the first such compound, silmitasertib, an CK II-selective ATP-competitive benzo[c][2,6]naphthyridine-8-carboxylic acid compound, has recently entered clinical evaluation (Table S29). ${ }^{173}$

MELK is an AMPK family kinase with several cellular functions related to survival and proliferation and it is overexpressed in many tumours. These functions appear to be especially important in cancer stem cells, i.e. undifferentiated cancer cells. The first clinical MELK inhibitor is OTS 167, a disubstituted (naphthyridin-3-yl)ethanone compound, whose binding mode was reported recently. ${ }^{174}$ Another clinical compound, the (indol-3-ylidene)methyl)pyrrole amcasertib, also targets (undisclosed) cancer stem cell pathway kinases (Table S29).

PIM. The proviral insertion in murine (PIM) lymphoma family of Ser/Thr kinases (PIM1-3) is expressed predominantly in haematopoietic cells and signals downstream from $A B L$, JAK2, and FLT3. PIM kinases contribute to the regulation of the cell cycle, apoptosis, and the proliferation and migration of cells. All three isoforms have oncogenic potential and are aberrantly expressed in various tumours. ${ }^{175}$ Although mouse knock-out of any of the three PIM kinases results in mild phenotypes, deficient PIM1 signalling is associated with cardiac function and PIM triple knock-out mice develop heart failure by 6 months. These observations may be relevant to the withdrawal of the first-in-class clinical PIM inhibitor SGI 1776 due to cardiotoxicity. ${ }^{176}$ Despite this observation it would appear that both PIM inhibitors currently undergoing clinical development, i.e. INCB 053914 and PIM 447 (Table S18) are pan-selective PIM inhibitors. PIM kinases are somewhat unusual insofar as they recognise ATP, as well as most inhibitors, in a manner that does not involve the typical H-boding interactions between the kinase hinge region and the ligand, due to the presence of two Pro residues in the PIM hinge, which imparts an atypical conformation to this region. ${ }^{177}$ This unusual recognition mode may explain the high kinome-wide selectivity of compounds such as PIM 447. 
Mitogen-activated protein kinase pathway kinases. The RAS-RAF-MEK-ERK MAPK pathway is one of the main signalling pathways that cancer cells use for the purposes of proliferative and survival advantages. Activation of the MAPK pathway, through activating mutations in either NRAS or BRAF, is particularly common in melanoma. ${ }^{178}$ Below we shall discuss clinical RAF (Table S19) and MEK inhibitors (Table S20), which have recently been joined by the first ERK inhibitors (Table S21).

Currently there are two RAF kinase inhibitors approved for the treatment of unresectable or metastatic melanoma with BRAF V600E mutation, vemurafenib and dabrafenib. Both compounds display selectivity for V600E BRAF over other kinases, and to a lesser extent over wild-type RAF kinase isoforms. ${ }^{179,180}$ With these compounds, inhibition of the MAPK pathway is specific to tumour cells with mutant $B R A F$ and this is likely due to their selectivity. In the majority of melanoma patients treated with vemurafenib or dabrafenib significant tumour regression and progression-free survival have been observed, a result that compares favourably with previously available therapies for metastatic melanoma. ${ }^{178}$

Neither agent is indicated for the treatment of patients with wild-type BRAF melanoma due to the potential risk of tumour promotion, and both were approved concurrently with assays for the detection of BRAF V600E mutations. This contra-indication is due to paradoxical activation of the MAPK pathway by RAF inhibitors in cells with wild-type BRAF. MAPK pathway activation occurs as a result of RAS-dependent MEK phosphorylation by homoand hetero-dimerised RAF isoforms. In mutant BRAF melanoma cells, in which MEK activation is driven by BRAF, RAF inhibition is effective because of increased affinity of BRAF V600E for ATP and lack of CRAF activation. Paradoxical MAPK pathway activation in cells with normal $B R A F$, where RAS signalling occurs predominantly through CRAF, is thought to arise due to binding of type-I RAF inhibitors to wild-type RAF isoforms, which leads to their loss of auto-inhibition in a manner that is independent of kinase inhibition, enhanced RAF dimerisation, membrane localisation, and eventually RAS-dependent MEK activation. ${ }^{181,182}$ The same phenomenon, i.e. paradoxical MAPK pathway activation in healthy keratinocytes, is probably responsible for the significant cutaneous toxicities observed with RAF inhibitors. ${ }^{183}$

Despite the approval of vemurafenib and dabrafenib, a whole range of additional RAF kinase inhibitors are currently being developed in advanced melanoma and other solid tumours (Table S19). ${ }^{184}$ The first RAF inhibitor studied in melanoma, however, was sorafenib, a type-II MTKI with pan-RAF activity. This compound, which also has activity against several CSF1/PDGF receptor subfamily kinases (Table S5), was being developed -and is now approved in-advanced RCC at the time when the BRAF V600E mutation, which is present in over half of all malignant melanomas, was first discovered. ${ }^{185}$ Despite several clinical trials of sorafenib in melanoma, it was not possible to show significant patient benefit, 
however. Presumably this was because at the maximum tolerated dose, defined by toxicities emanating from inhibition of kinases other than RAF, there was insufficient MAPK pathway inhibition. ${ }^{178}$

The first MEK inhibitor (Table S20) approval was trametinib in the treatment of patients with unresectable or metastatic melanoma with BRAF V600E or BRAF V600K mutation as detected by an FDA-approved test. This offers another treatment modality in this disease, although it is not as yet clear what the best treatment options with RAF and MEK inhibitors are. ${ }^{186}$ Nevertheless, acquired resistance to RAF inhibitors occurs frequently and through multiple genetic mechanisms, which suggests that simultaneous inhibition of MEK and mutant RAF kinases may be a better strategy in terms of overall efficacy, prevention of MEKdriven acquired resistance, and modulation of toxicity emanating from paradoxical MAPKpathway activation with RAF-inhibitor monotherapy. ${ }^{187}$ On this basis cobimetinib was recently approved for the treatment of mutant BRAF melanoma in combination with vemurafenib.

The selectivity of trametinib and cobimetinib, as well as other MEK inhibitors under clinical development, ${ }^{188}$ including rafametinib, selumetinib, pimasertib, PD-0325901, binimetinib, RG 7304, and TAK 733, is probably because these compounds target a non-conserved allosteric site in MEK1 and MEK2 (Figure 13).

Although treatment of advanced melanoma with both RAF and MEK inhibitors has been observed to be effective initially, resistance to both inhibitor types eventually occurs. ${ }^{189}$ The main mechanisms of acquired resistance appear to be reactivation of the MAPK pathway in a number of ways, as well as compensatory signalling through the parallel PI3K pathway. ${ }^{178}$ The latter observation has led to the initiation of clinical trials in melanoma and other solid tumours, where RAF/MEK and PI3K inhibitors are used in combination. ${ }^{190}$ Direct targeting of ERK1/2 in order to block signalling through the RAS-RAF-MEK-ERK pathway has lagged behind the upstream inhibition strategies, but may be of benefit in several ways. ${ }^{191}$ Thus it has been demonstrated that dual pharmacological inhibition of MEK and ERK can be synergistic by inhibiting the emergence of resistance and overcoming acquired resistance to MEK inhibitors. ${ }^{192}$ There are currently three ERK1/2 inhibitors under clinical evaluation (Table S21), the most advanced being ulixertinib. Both ulixertinib and GDC 0994 contain the $N$-((1S)-2-hydroxy-1-phenylethyl)formamide substructure, whereas the chemical structure of CC 90003 has not been disclosed.

p38 MAPK. Several different MAPK signalling pathways are used by cells to respond to extracellular stimuli. Apart from the RAS-RAF-MEK-ERK we have just discussed, another important MAPK pathway is the p38 MAPK pathway, which mediates stress responses and responses to cytokines, chemokines, hormones, and growth factors. The four p38 Ser/Thr kinase isoforms p38a (MAPK14), p38ß (MAPK11), p38ס (MAPK13), and p38y (MAPK12) 
can be activated in a number of ways involving both posttranslational modification and protein interactions, thus integrating many different signals. In turn the p38 kinases have numerous downstream targets and can activate different transcriptional programmes in a spatially and temporally controlled manner. ${ }^{193}$

p38 MAPK is pursued mainly as a drug target in chronic inflammatory disorders, especially p38a, which is over-activated in inflamed tissues. Many different p38 MAPK inhibitors are being developed in such disorders, including rheumatoid arthritis, atherosclerosis, and asthma. ${ }^{194,195}$ However, p38 MAPKs also play important roles in the responses of cancer cells to oncogenic stress, radiation, and chemotherapy and altered expression of p38 MAPKs is frequently observed in tumours, where they promote tumourigenesis. ${ }^{196}$ Currently two structurally related p38 MAPK inhibitors are under clinical investigation in oncology indications: ralimetinib and pexmetinib (Table S22).

MNK. The Ser/Thr kinases MNK1 and MNK2 are activated through the mitogen- and stressactivated MAP kinase pathways and they phosphorylate the eukaryotic translation initiation factor 4E (eIF4e). Genetic studies indicate that whereas MNK activity is required for eIF4Emediated oncogenic cell transformation, such activity is dispensable for normal development. ${ }^{197}$ Until recently very few MNK inhibitors were known but selective type-I/II and type-VI MNK inhibitors have now been reported. ${ }^{198,199}$ The first MNK inhibitor to reach phase-I clinical trial is BAY 1143269 (Table S29; undisclosed structure).

Cyclin-dependent kinases (Table S23). The CDKs comprise a large group of Ser/Thr kinases involved predominantly in the control of the cell cycle and the regulation of transcription. Recruitment into and progression through the cell cycle are tightly regulated and the interphase CDKs control the first gap phase $\left(\mathrm{G}_{1}\right.$; CDK4, CDK6, CDK1, CDK2) by activating E2F-responsive genes required for entry to the DNA synthesis phase (S-phase), as well as the second gap phase $\left(\mathrm{G}_{2} ; \mathrm{CDK} 1, \mathrm{CDK} 2\right)$ by activating the FoxM1 gene programme required for execution of mitosis. Similarly, the different phases of RNA polymerase II (RNAPII)-based transcription are regulated by CDKs. Here sequential phosphorylation of the C-terminal domain of RNAPII occurs: by CDK7 and CDK8 in transcription initiation, and by CDK9 in RNA elongation. Additionally, CDK activity is required (at least CDK11) for RNA processing (splicing). ${ }^{200}$ However, our understanding of CDK biology is still incomplete due to extensive functional overlap and redundancy between the many CDK-cyclin pairs, not only in the cell cycle and RNAPII transcription, but also between the two.

Because cancer is characterised by uncontrolled cell division, interference with CDKs as key regulators of the cell cycle appears attractive as a therapeutic strategy. However, the complexity of CDK-cyclin biology has confounded interpretable genetic target validation of CDKs. As a result uncertainty exists about which CDK function(s) should be blocked to achieve effective and specific anticancer activity in a therapeutic setting, and this has long 
beset the development of CDK inhibitor drugs. A large number of CDK inhibitors had been trialled clinically in the past - mostly with disappointing results. ${ }^{201}$

Nevertheless, the first CDK inhibitor, palbociclib, has now been granted FDA approval for use in combination with letrozole (a non-steroidal aromatase inhibitor) in ER-positive, HER2-negative metastatic breast cancer. Palbociclib is a highly CDK4/6-selective agent and preclinical studies with this and several other CDK4/6-selective compounds show anticancer activity in a range of models, with this activity being contingent on the presence of functional retinoblastoma protein ( $\mathrm{pRb}$; the CDK4/6 phosphorylation substrate), and leading to cytostatic $G_{1}$ arrest. Rapidly reversible neutropenia is the main dose-limiting toxicity of palbociclib, as one would expect on mechanistic grounds. ${ }^{201}$

Other CDK inhibitors that remain under clinical development include two more CDK4/6selective agents: ribociclib, abemaciclib, as well as number of pan-CDK-selective compounds: dinaciclib, AT 7519, milciclib, roniciclib, and CYC 065. Additionally, MTKIs that may be classed as CDK-inhibitors are TG 02 and RGB 286638.

The kinase inhibitor that probably has a longer clinical development history than any other is alvocidib (flavopiridol). This compound has been difficult to develop due to target promiscuity and limitations in formulation and disposition, but marked efficacy was observed some time ago in CLL, and this activity was ascribed to potent CDK9 inhibition by alvocidib. CLL -and many other cancers- are known selectively to depend on efficient RNA synthesis in order to support anti-apoptotic signalling, which in turn requires CDK9 activity. ${ }^{202} \mathrm{Al}-$ vocidib has now re-entered clinical development, based on promising activity in AML, also likely due to potent CDK9 inhibition. The first selective CDK9 inhibitor, BAY 1143572, has also now entered clinical trials. This compound contains the unusual sulfoximine solubilising substructure. ${ }^{203}$

Aurora kinases. ARKs are a group of mitotic Ser/Thr kinases and in humans there are three homologues. ARK-A regulates mitotic entry, spindle assembly, alignment of metaphase chromosomes, and completion of cytokinesis. ARK-B and ARK-C, on the other hand, are chromosomal passenger proteins predominantly involved in chromosomal bi-orientation. ARKs play important roles in the maintenance of genetic stability and their aberrant expression leads to genomic instability or aneuploidy. Because ARKs are overexpressed in many cancers, thus generating aggressive tumours, they are regarded by many as attractive targets for cancer therapy. ${ }^{204}$

There is an on-going debate in the field about what ARK selectivity is optimal in terms of efficacy and on-target toxicity. Currently six ARK inhibitors (Table S24) with different selectivity profiles are being trialled in a variety of solid tumours and blood cancers. Compounds selective for ARK-A are alisertib and TAS 119, whereas barasertib is selective for ARK-B. Danusertib is a selective pan-ARK inhibitor. ENMD 2076 and ilorasertib, on the 
other hand, are multi-targeted ARK inhibitors. An answer regarding desirable selectivity may therefore emerge. At this stage, however, this questions remains open.

Whereas some therapeutic effects have been seen with most experimental ARK inhibitor drugs, it would appear that all of them suffer the same limitations as classical antimitotic agents, i.e. cytostatic or cytotoxic effects against proliferating cells only, without good discrimination between transformed and normal cells, which manifest clinically as neutropenia and other haematological toxicities. Interestingly, several ARK inhibitors have been found to be effective against imatinib- and $2^{\text {nd }}$-generation Tyr kinase inhibitor-resistant forms of the BCR-ABL kinase, especially the T315I mutant form. Some ARK inhibitors are now being tested clinically in patients with imatinib-resistant CML. ${ }^{205}$

Polo-like kinases. PLK1 is one of four related Ser/Thr kinases associated with cell proliferation, especially in the mitotic phase of the cell cycle. Most cancers exhibit higher PLK1 expression than corresponding normal tissues, and PLK1 inhibition results in $\mathrm{G}_{2}-\mathrm{M}$ phase arrest, activation of the mitotic checkpoint, spindle dysfunction, and apoptosis in cancer cells. To date several PLK inhibitors have entered clinical evaluation, so far with disappointing results in terms of efficacy and with neutropenia as the most prominent toxicity. ${ }^{206}$ The most advanced experimental PLK inhibitor drug is volasertib, a potent and selective PLK1 inhibitor with pronounced anti-tumour activity in preclinical models, including taxane-resistant colorectal cancer. One of the possible reasons for the limited anticancer activity observed with this agent in humans may be its limited bioavailability in tumours compared to normal tissues. ${ }^{207}$ Using a genome-wide synthetic lethality screen it was shown recently that KRAS-driven tumours are selectively sensitive to PLK1 inhibition. ${ }^{208}$ This finding might be used profitably to guide patient selection in efficacy trials of experimental PLK1 inhibitor drugs. PLK4, which is involved in centriole duplication, has not as yet received significant attention as an oncology target, but a selective PLK4 inhibitor, CFI 400945, has recently entered clinical evaluation (Table S25).

DNA damage response pathway kinases. The PI3K-related kinase (PIKK) family includes ATM, ATR, DNA-PK, hSMG1, mTOR (discussed above under PI3K pathway inhibitors), and TRRAP, all large proteins that contain a C-terminal kinase domain closely related to the lipid kinase PI3K, but which function as Ser/Thr kinases, whereas CHK1 and CHK2 are Ser/Thr kinases in the CAMK family (NIM1 subfamily). Several PIKKs, including ATM, ATR, and DNA-PK, as well as the CHK kinases, are implicated in DNA replication and damage checkpoint pathways.

The DNA damage response helps cells to maintain genomic stability and the two main signalling routes are the ATM-CHK2 pathway, which controls DNA double-strand break repair, and the ATR-CHK1 pathway, which responds primarily to single-stranded DNA. DNA-PK, on the other hand, plays a role in DNA repair by non-homologous end joining. ATM and ATR act on their CHK substrates, which in turn promote cell-cycle arrest to allow 
time for repair, as well as on numerous other components involved in DNA repair, apoptosis, and the cell cycle. Since efficient DNA repair allows cancer cells to tolerate not only oncogenic replication stress, but also exogenous genotoxic stress from radio- and chemotherapy, the DNA response pathway may represent a promising target for cancer therapy. At least ATR and CHK1 inhibitors are believed to be potentially useful for monotherapy of certain cancers, e.g. those with p53 and other DNA damage response deficiencies, whereas ATM, ATR, CHKs, and DNA-PK inhibitors are all potentially valuable as radio- and chemosensitising agents. ${ }^{209}$

Although a number of $\mathrm{CHK}$ inhibitors had been clinically evaluated in the past, ${ }^{210}$ currently only the CHK1 inhibitors prexasertib and GDC 0575 remain under active development (Table S26). The first selective ATR inhibitors to enter the clinic are VX 970, VX 803, and AZD 6738 (Table S27). Additionally, the ATM inhibitor AZD 0156 (Table S29) and the DNA-PK inhibitors VX 984 and M 3814 (Table S28) are in early clinical trials. Finally the first-inclass WEE1 inhibitor AZD 1775 (Table S29) should be mentioned here, as WEE1 is another kinase associated with cell cycle checkpoint control following DNA damage.

CDC7. CDC7 is a Ser/Thr kinase that is involved in regulation of the S-phase and mitosis during the cell cycle. CDC7 inhibition has been reported to block DNA replication without activation of the S-phase checkpoint and directly to induce cellular apoptosis in transformed cells but not in normal cells. ${ }^{211} \mathrm{~A}$ number of CDC7 inhibitors have been reported and several have undergone early clinical evaluation. ${ }^{212}$ However, at present the only CDC7 inhibitor in the clinic is TAK 931 (Table S29; undisclosed structure).

\section{Medicinal chemistry}

Of the 231 (at the time of writing) clinical kinase inhibitor anticancer drugs, chemical structures for 172 are in the public domain. These molecules display structural and physicochemical properties as summarised and compared to those of all currently approved smallmolecule drugs in Table 1.

In terms of physicochemical properties, it can be seen that on average oncology kinase inhibitors are significantly larger (by almost a third; $M_{r}$ ) and more lipophilic (by around two thirds; $\log \mathrm{P}, \log \mathrm{D}$ ) than small-molecule drugs in general, and they have much lower (predicted) intrinsic water solubility (by almost half; logS). Increased lipophilicity and lower solubility stem predominantly from relatively abundant aliphatic and aromatic systems (nAr) present in oncology kinase inhibitors. Furthermore, kinase inhibitors are comparatively more flexible (nRot) and contain more polar functionalities for $\mathrm{H}$-bonding interactions (TPSA, HBA, HBD) than approved drugs in general. Structurally, kinase inhibitors on average contain more cyclic systems (nRing, nAr) but fewer chiral centres ( $\mathrm{nChir}$ ) than other drugs, presumably because kinase inhibitors, unlike many other drugs, are less frequently derived from natural products. 
Correlation of $\log \mathrm{P}$ and logS (Figure 14) shows that most of the kinase inhibitor drugs are expected to be highly permeable but poorly soluble, some of them with exceptionally low (predicted) intrinsic aqueous solubility. With very few exceptions kinase inhibitor drugs are being developed as immediate release products for administration by the oral route and while the physicochemical properties in Table 1 are consistent with oral bioavailability, ${ }^{213,214}$ average $M_{\mathrm{r}}$ and logs suggest an average thermodynamic aqueous solubility of about $2 \mu \mathrm{g} / \mathrm{mL}$ ( $75 \mu \mathrm{g} / \mathrm{mL}$ for all approved drugs), which is only expected to be consistent with very low clinical doses, and provided permeability is high. ${ }^{215}$ For many oncology kinase inhibitors absorption is likely to be variable and in vivo drug dissolution rate-limiting. It can therefore be expected that many kinase inhibitor drugs will be challenging to develop from a formulation viewpoint.

As can be seen from Figure 15, both the maximum and average structural dissimilarity amongst oncology kinase inhibitors is significantly lower than that of all approved drugs, indicating that many kinase inhibitors, which generally target the conserved ATP-binding site, are pharmacophorically related. As expected, dissimilarity of compounds that are targeted to subgroups or individual kinases is diminished compared to that of all experimental kinase inhibitors. The groups of kinase inhibitors with the highest and lowest average structural dissimilarity are the PI3K and CSF-1/PDGF inhibitors on the one hand, and the BCR-ABL inhibitors on the other.

Another way of looking at structural similarity of oncology kinase inhibitor drugs is to assess the frequency at which common substructures occur in these compounds (Figure 16). This shows that by far the most common substructure is the formanilide group (31 instances). This is probably not a distinguishing structural feature of kinase inhibitors, as this group occurs frequently in many different drug classes. The next most frequent substructure, however, is the quinazoline group (14 instances), a commonly used kinase inhibitor core, which occurs in several approved drugs and many experimental agents, especially in EGFR inhibitors. Other substructures that occur more than three times in the 172 oncology kinase inhibitors are the ethoxybenzene (11), 2-anilinopyrimidine (10), benzamide (8), indole (8), benzimidazole (5), chromene (4), 4-(pyrimidinyl-4-yl)morpholine (4), (3-fluorophenyl)methanamine (4), 2-phenylethylamine (4), and N,4-dimethylpyrimidin-2-amine (4). Again some of these substructures are not specific to kinase inhibitors but it is notable that 3 of these substructures contain the pyrimidine heterocycle. Of the substructures that occur twice or three times a number of $\mathrm{N}$-containing bicyclic heterocycles, including purines, 3-methyleneoxindoles, pyrrolo[2,3- $d$ ]pyrimidines are especially associated with kinase inhibitors.

Whereas kinase inhibitor lead compounds were frequently discovered using high throughput screening in the past, a much more common current strategy is structure-based redesign of known kinase inhibitor templates, as is evident from the preceding similarity and 
property comparisons of clinical kinase inhibitors. More recently fragment-based drug discovery has started to play an increasingly important role in the design of kinase inhibitors and the first approved kinase inhibitor arrived at by this strategy is vemurafenib. ${ }^{216} \mathrm{~A}$ more recent example is AZD 5363 (Figure 17).

Using a virtual screen of fragment compounds $\left(M_{r}<250\right)$ against a model of AKT based on a crystallographic complex of an active form of that protein, followed by crystallography of putative binding fragments with a PKA-AKT chimera construct, 7-azaindole was identified as an AKT hinge-binding fragment (Figure 17a). ${ }^{217}$ The binding mode of the isoquinoline-5-sulfonamide AKT inhibitor CCT 077373 discovered earlier ${ }^{218}$ suggested that determinants for AKT inhibition activity other than hinge binding were a basic amine group interacting with acidic groups in the ribose-binding regions, as well as a lipophilic group (chlorophenyl) interacting with the G-loop (Figure 17f). For synthetic ease the azaindole was replaced with purine, which was arylated at the 6-position to afford benzylamine intermediate 1 as one of the most active and ligand-efficient derivatives (Figure 17b). Replacement of the phenyl group in that intermediate with piperidine gave rise to compounds with improved polar interactions with the acidic residues in the ribose-binding region and intermediate 2 (Figure 17c) was identified as a compound with improved activity and excellent lipophilic ligand efficiency. ${ }^{219}$ However, this compound was too polar and lacked cellular activity. Polarity was modulated by replacement of the azaindole core with pyrrolo[2,3-d]pyrimidine, repositioning of the primary amine group, and extension with a 4chlorophenyl group to give rise to intermediate 3 (Figure 17d), a highly potent AKT inhibitor with well-balanced physicochemical properties. ${ }^{219,220}$. Intermediate 3 was subsequently further elaborated in terms of kinase selectivity, hERG activity, and in vivo disposition properties to afford AZD 5363 (Figure 17e)..$^{221}$

\section{Conclusions}

Kinase inhibitor discovery is a comparatively recent medicinal chemistry endeavour and has progressed almost exponentially over the last 25 years. Whereas early kinase inhibitor approvals were for MTKIs, the preponderance of more selective agents has increased gradually and now predominates. The advent of kinome-wide kinase assays and associated molecular pharmacology technologies, and the ever-expanding knowledge of how kinase inhibitors engage their molecular targets at the atomic level, now permit the rational design and validation of highly selective agents. This, together with modern clinical investigation strategies incorporating patient diagnosis, prognosis, and stratification based on genetic and mechanistic biomarkers, will continue to expand the oncologist's arsenal of highly specific kinase inhibitor drugs and will hopefully permit truly effective, modular, personalised treatments of cancers with less reliance on toxic chemotherapy. Because in general highly target-selective agents are also highly specific in vivo, it is hoped that progress can be 
made to optimise the therapeutic margin (therapeutic versus toxic effects) of kinase inhibitor drugs, not only in oncology, but also in other therapeutic indications with unmet medical need. Based on current information it is likely that this may not be achievable in many cases with inhibitors that target the catalytic activity of kinases, except in those cases where a particular cancer is addicted completely to the activity of a given kinase. In principle pharmacological modulation of kinase activities is also possible by allosteric means, as we have seen. This is especially attractive if the possibility of such allostery arises in a manner that is specific for a particular context in terms of kinase environment and location, e.g. through specific disease-associated interactions of a kinases, but such approaches remain challenging. Maximisation of kinase inhibitor efficacy by these or other means is highly important in order to avoid emergence of drug resistance, which is a feature of many current kinase inhibitors, especially those with high target selectivity. As we have seen, the first kinase inhibitors for inflammatory disease have now been approved and several kinase inhibitors are under clinical investigation in indications other than cancer. A major challenge will be to apply what we have learned from oncology kinase inhibitors to other major disorders that currently lack effective therapies, such as neurodegeneration and (drug-resistant) microbial and viral infections.

Abbreviations. For kinase abbreviations refer to Table S1. Other abbreviations: ALCL, anaplastic large cell lymphoma; ALL, acute lymphocytic leukaemia; AML, acute myelogenous leukaemia; BCR, B-cell antigen receptor; CLL, chronic lymphocytic leukaemia;

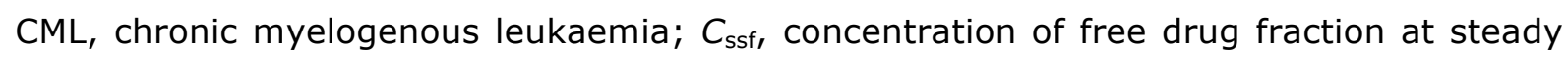
state; DLBCL, diffuse large B-cell lymphoma; DFSP, dermatofibroma sarcoma protuberans; FDA, U.S. Food and Drug Administration; $f o_{t}$, receptor occupancy as a function of time; GIST, gastrointestinal stromal tumour; IPF, idiopathic pulmonary fibrosis; MTC, medullary thyroid cancer; MTKI, multi-targeted kinase inhibitor; NHL, non-Hodgkin's lymphoma; NSCLC, non-small cell lung cancer; $\mathrm{PH}$, pleckstrin homology; $\mathrm{PIP}_{3}$, phosphatidylinositol3,4,5-trisphosphate; RCC, renal cell carcinoma; RNAPII, RNA polymerase II; SI, selectivity index.

\section{References}

1. Budzyn K, Marley PD, Sobey CG. Targeting Rho and Rho-kinase in the treatment of cardiovascular disease. Trends Pharmacol Sci 2006;27(2):97-104.

2. Sasaki Y, Suzuki M, Hidaka H. The novel and specific Rho-kinase inhibitor (S)-(+)-2-methyl-1-[(4methyl- 5-isoquinoline)sulfonyl]-homopiperazine as a probing molecule for Rho-kinase-involved pathway. Pharmacol Ther 2002;93(2-3):225-232.

3. Rath N, Olson MF. Rho-associated kinases in tumorigenesis: re-considering ROCK inhibition for cancer therapy. EMBO Rep 2012;13(10):900-908.

4. O'Dell KM, Rummel AE. Tofacitinib: a novel oral Janus kinase inhibitor for rheumatoid arthritis.

Formulary 2012;47(10):350, 353-358.

5. Grant S. Therapeutic Protein Kinase Inhibitors. Cell Mol Life Sci 2009;66(7):1163-1177. 
6. Mazzei ME, Richeldi L, Collard HR. Nintedanib in the treatment of idiopathic pulmonary fibrosis. Ther Adv Respir Dis 2015;9(3):121-129.

7. Reichert JM, Wenger JB. Development trends for new cancer therapeutics and vaccines. Drug Discov Today 2008;13(1-2):30-37.

8. Walker I, Newell H. Do molecularly targeted agents in oncology have reduced attrition rates? Nat Rev Drug Discov 2009;8(1):15-16.

9. Capdeville R, Buchdunger E, Zimmermann J, Matter A. Glivec (STI571, imatinib), a rationally developed, targeted anticancer drug. Nat Rev Drug Discov 2002;1(7):493-502.

10. Roth BL, Sheffler DJ, Kroeze WK. Magic shotguns versus magic bullets: selectively non-selective drugs for mood disorders and schizophrenia. Nat Rev Drug Discov 2004;3(4):353-359.

11. Goldstein DM, Gray NS, Zarrinkar PP. High-throughput kinase profiling as a platform for drug discovery. Nat Rev Drug Discov 2008;7(5):391-397.

12. Patricelli MP, Szardenings AK, Liyanage M, Nomanbhoy TK, Wu M, Weissig H, Aban A, Chun D, Tanner $\mathrm{S}$, Kozarich JW. Functional interrogation of the kinome using nucleotide acyl phosphates. Biochemistry 2007;46(2):350-358.

13. Shoshan MC, Linder S. Target specificity and off-target effects as determinants of cancer drug efficacy. Expert Opin Drug Metabol Toxicol 2008;4(3):273-280.

14. Csermely $\mathrm{P}$, Agoston $\mathrm{V}$, Pongor $\mathrm{S}$. The efficiency of multi-target drugs: the network approach might help drug design. Trends Pharmacol Sci 2005;26(4):178-182.

15. Hopkins AL, Mason JS, Overington JP. Can we rationally design promiscuous drugs? Curr Opin Struct Biol 2006;16(1):127-136.

16. Jenwitheesuk E, Horst JA, Rivas KL, Van Voorhis WC, Samudrala R. Novel paradigms for drug discovery: computational multitarget screening. Trends Pharmacol Sci 2008;29(2):62-71.

17. Roskoski R. Classification of small molecule protein kinase inhibitors based upon the structures of their drug-enzyme complexes. Pharmacol Res 2016;103:26-48.

18. Zhang J, Yang PL, Gray NS. Targeting cancer with small molecule kinase inhibitors. Nat Rev Cancer 2009;9(1):28-39.

19. Knight ZA, Shokat KM. Features of selective kinase inhibitors. Chem Biol 2005;12(6):621-637.

20. Cheng $\mathrm{Y}-\mathrm{C}$, Prusoff WH. Relation between the inhibition constant IK1) and the concentration of inhibitor which causes fifty per cent inhibition (I50) of an enzymic reaction. Biochem Pharmacol 1973;22(23):3099-3108.

21. Garuti L, Roberti M, Bottegoni G. Non-ATP Competitive Protein Kinase Inhibitors. Curr Med Chem 2010;17(25):2804-2821.

22. Tummino PJ, Copeland RA. Residence Time of Receptor-Ligand Complexes and Its Effect on Biological Function. Biochemistry 2008;47(20):5481-5492.

23. Bradshaw JM, McFarland JM, Paavilainen VO, Bisconte A, Tam D, Phan VT, Romanov S, Finkle D, Shu J, Patel V, Ton T, Li X, Loughhead DG, Nunn PA, Karr DE, Gerritsen ME, Funk JO, Owens TD, Verner E, Brameld KA, Hill RJ, Goldstein DM, Taunton J. Prolonged and tunable residence time using reversible covalent kinase inhibitors. Nat Chem Biol 2015;11(7):525-531.

24. Copeland RA. The drug-target residence time model: a 10-year retrospective. Nat Rev Drug Discov 2016;15(2):87-95

25. Arteaga CL. Molecular Therapeutics: Is One Promiscuous Drug against Multiple Targets Better than Combinations of Molecule-specific Drugs? Clin Cancer Res 2003;9(4):1231-1232.

26. Faivre S, Djelloul S, Raymond E. New paradigms in anticancer therapy: targeting multiple signaling pathways with kinase inhibitors. Semin Oncol 2006;33(4):407-420.

27. de Jonge MJA, Verweij J. Multiple targeted tyrosine kinase inhibition in the clinic: all for one or one for all? Eur J Cancer 2006;42(10):1351-1356.

28. Van Etten RA. Pathogenesis and treatment of $\mathrm{Ph}+$ leukemia: recent insights from mouse models. Curr Opin Hematol 2001;8(4):224-230.

29. Druker BJ. Imatinib: Paradigm or anomaly? Cell Cycle $2004 ; 3(7): 833-835$.

30. Weinstein IB, Joe AK. Mechanisms of disease: Oncogene addiction--a rationale for molecular targeting in cancer therapy. Nat Clin Pract Oncol 2006;3(8):448-457.

31. Sharma SV, Settleman J. Oncogene addiction: setting the stage for molecularly targeted cancer therapy. Genes Dev 2007;21(24):3214-3231.

32. Comoglio PM, Giordano S, Trusolino L. Drug development of MET inhibitors: targeting oncogene addiction and expedience. Nat Rev Drug Discov 2008;7(6):504-516.

33. Daub H, Specht K, Ullrich A. Strategies to overcome resistance to targeted protein kinase inhibitors. Nat Rev Drug Discov 2004;3(12):1001-1010.

34. Engelman JA, Janne PA. Mechanisms of acquired resistance to epidermal growth factor receptor tyrosine kinase inhibitors in non-small cell lung cancer. Clin Cancer Res 2008;14(10):2895-2899.

35. D'Amato V, Raimondo L, Formisano L, Giuliano M, De Placido S, Rosa R, Bianco R. Mechanisms of lapatinib resistance in HER2-driven breast cancer. Cancer Treat Rev 2015;41(10):877-883.

36. Fojo T. Multiple paths to a drug resistance phenotype: mutations, translocations, deletions and amplification of coding genes or promoter regions, epigenetic changes and microRNAs. Drug Resist Update 2007; 10(1-2):59-67.

37. Duesberg P, Stindl R, Hehlmann R. Origin of multidrug resistance in cells with and without multidrug resistance genes: chromosome reassortments catalyzed by aneuploidy. Proc Natl Acad Sci USA 2001;98(20):11283-11288.

38. Duesberg P, Li R, Sachs R, Fabarius A, Upender MB, Hehlmann R. Cancer drug resistance: The central role of the karyotype. Drug Resist Update 2007;10(1-2):51-58. 
39. Kerkela R, Grazette L, Yacobi R, Iliescu C, Patten R, Beahm C, Walters B, Shevtsov S, Pesant S, Clubb FJ, Rosenzweig A, Salomon RN, Van Etten RA, Alroy J, Durand JB, Force T. Cardiotoxicity of the cancer therapeutic agent imatinib mesylate. Nat Med 2006;12(8):908-916.

40. Berman E, Nicolaides M, Maki RG, Fleisher M, Chanel S, Scheu K, Wilson BA, Heller G, Sauter NP. Altered bone and mineral metabolism in patients receiving imatinib mesylate. $\mathrm{N}$ Engl J Med 2006;354(19):2006-2013.

41. Bianchini D, Jayanth A, Chua YJ, Cunningham D. Epidermal growth factor receptor inhibitor-related skin toxicity: mechanisms, treatment, and its potential role as a predictive marker. Clin Colorectal Canc 2008;7(1):33-43.

42. Robert C, Mateus C, Spatz A, Wechsler J, Escudier B. Dermatologic symptoms associated with the multikinase inhibitor sorafenib. J Am Acad Dermatol 2009;60(2):299-305.

43. Barrick CJ, Yu M, Chao H-H, Threadgill DW. Chronic pharmacologic inhibition of EGFR leads to cardiac dysfunction in C57BL/6] mice. Toxicol Appl Pharm 2008;228(3):315-325.

44. Force T, Krause DS, Van Etten RA. Molecular mechanisms of cardiotoxicity of tyrosine kinase inhibition. Nat Rev Cancer 2007;7(5):332-344.

45. Crouthamel M-C, Kahana JA, Korenchuk S, Zhang S-Y, Sundaresan G, Eberwein DJ, Brown KK, Kumar R. Mechanism and Management of AKT Inhibitor-Induced Hyperglycemia. Clin Cancer Res 2009;15(1):217-225.

46. De Klein A, Van KAG, Grosveld G, Bartram CR, Hagemeijer A, Bootsma D, Spurr NK, Heisterkamp N, Groffen J, Stephenson JR. A cellular oncogene is translocated to the Philadelphia chromosome in chronic myelocytic leukemia. Nature 1982;300(5894):765-767.

47. Kantarjian H, O'Brien S, Garcia-Manero G, Faderl S, Ravandi F, Jabbour E, Shan J, Cortes J. Very longterm follow-up results of imatinib mesylate therapy in chronic phase chronic myeloid leukemia after failure of interferon alpha therapy. Cancer 2012;118(12):3116-3122.

48. Quintas-Cardama A, Kantarjian H, Cortes J. Flying under the radar: the new wave of BCR-ABL inhibitors. Nat Rev Drug Discov 2007;6(10):834-848.

49. Morphy R. Selectively Nonselective Kinase Inhibition: Striking the Right Balance. J Med Chem 2010;53(4):1413-1437.

50. Keller-von Amsberg G, Koschmieder S. Profile of bosutinib and its clinical potential in the treatment of chronic myeloid leukemia. OncoTargets Ther 2013;6:99-106.

51. Mauro MJ. T315I, more or less, predicts for major molecular response: the devil is in the details! Haematologica 2013;98(5):665-666.

52. Parsons SJ, Parsons JT. Src family kinases, key regulators of signal transduction. Oncogene 2004;23(48): 7906-7909.

53. Nam H-J, Im S-A, Oh D-Y, Elvin P, Kim H-P, Yoon Y-K, Min A, Song S-H, Han S-W, Kim T-Y, Bang Y-J. Antitumor Activity of Saracatinib (AZD0530), a c-Src/Abl Kinase Inhibitor, Alone or in Combination with Chemotherapeutic Agents in Gastric Cancer. Mol Cancer Ther 2013;12(1):16-26.

54. Nygaard HB, Wagner AF, Bowen GS, Good SP, MacAvoy MG, Strittmatter KA, Kaufman AC, Rosenberg BJ, Sekine-Konno T, Varma P, Chen K, Koleske AJ, Reiman EM, Strittmatter SM, van Dyck CH. A phase Ib multiple ascending dose study of the safety, tolerability, and central nervous system availability of AZD0530 (saracatinib) in Alzheimer's disease. Alzheimers Res Ther 2015;7(1):DOI 10.1186/s1319513015-10119-13190.

55. Zhang S, Yu D. Targeting Src family kinases in anti-cancer therapies: turning promise into triumph. Trends Pharmacol Sci 2012;33(3):122-128.

56. Noren NK, Foos G, Hauser CA, Pasquale EB. The EphB4 receptor suppresses breast cancer cell tumorigenicity through an Abl-Crk pathway. Nat Cell Biol 2006;8(8):815-825.

57. Fraser C, Dawson JC, Dowling R, Houston DR, Weiss JT, Munro AF, Muir M, Harrington L, Webster SP, Frame MC, Brunton VG, Patton EE, Carragher NO, Unciti-Broceta A. Rapid Discovery and StructureActivity Relationships of Pyrazolopyrimidines That Potently Suppress Breast Cancer Cell Growth via SRC Kinase Inhibition with Exceptional Selectivity over ABL Kinase. J Med Chem 2016;59(10):46974710.

58. Baselga J, Swain SM. Novel anticancer targets: revisiting ERBB2 and discovering ERBB3. Nat Rev Cancer 2009;9(7):463-475.

59. Lynch TJ, Bell DW, Sordella R, Gurubhagavatula S, Okimoto RA, Brannigan BW, Harris PL, Haserlat SM, Supko JG, Haluska FG, Louis DN, Christiani DC, Settleman J, Haber DA. Activating Mutations in the Epidermal Growth Factor Receptor Underlying Responsiveness of Non-Small-Cell Lung Cancer to Gefitinib. New Engl J Med 2004;350(21):2129-2139.

60. Burotto M, Manasanch EE, Wilkerson J, Fojo T. Gefitinib and erlotinib in metastatic non-small cell lung cancer: a meta-analysis of toxicity and efficacy of randomized clinical trials. Oncologist 2015;20(4):400-410.

61. Moy B, Kirkpatrick P, Kar S, Goss P. Lapatinib. Nat Rev Drug Discovery 2007;6(6):431-432.

62. Tan C-S, Gilligan D, Pacey S. Treatment approaches for EGFR-inhibitor-resistant patients with nonsmall-cell lung cancer. Lancet Oncol 2015;16(9):e447-e459.

63. Krop IE. Lessons from breast cancer trials of HER2-kinase inhibitors. Lancet Oncol 2016;17(3):267268.

64. Nagasawa J, Mizokami A, Koshida K, Yoshida S, Naito K, Namiki M. Novel HER2 selective tyrosine kinase inhibitor, TAK-165, inhibits bladder, kidney and androgen-independent prostate cancer in vitro and in vivo. Int J Urol 2006;13(5):587-592.

65. Cheng H, Nair SK, Murray BW. Recent progress on third generation covalent EGFR inhibitors. Bioorg Med Chem Lett 2016;26(8):1861-1868.

66. Sanderson K. Irreversible kinase inhibitors gain traction. Nat Rev Drug Discov 2013;12(9):649-651. 
67. Singh J, Petter RC, Baillie TA, Whitty A. The resurgence of covalent drugs. Nat Rev Drug Discov 2011;10(4):307-317.

68. Solca F, Dahl G, Zoephel A, Bader G, Sanderson M, Klein C, Kraemer O, Himmelsbach F, Haaksma E, Adolf GR. Target binding properties and cellular activity of afatinib (BIBW 2992), an irreversible ErbB family blocker. J Pharmacol Exp Ther 2012;343(2):342-350.

69. Hsieh AC, Moasser MM. Targeting HER proteins in cancer therapy and the role of the non-target HER3. $\mathrm{Br}$ J Cancer 2007;97(4):453-457.

70. Greig SL. Osimertinib: First Global Approval. Drugs 2016;76(2):263-273.

71. Minguet J, Smith KH, Bramlage P. Targeted therapies for treatment of non-small cell lung cancerRecent advances and future perspectives. Int J Cancer 2016;138(11):2549-2561.

72. Jackson AL, Davenport SM, Herzog TJ, Coleman RL. Targeting angiogenesis: vascular endothelial growth factor and related signaling pathways. Transl Cancer Res 2015;4(1):70-83.

73. Heldin C-H. Targeting the PDGF signaling pathway in tumor treatment. Cell Commun Signal 2013;11:97-97.

74. Garcia-Echeverria C, Traxler P, Evans DB. ATP site-directed competitive and irreversible inhibitors of protein kinases. Med Res Rev 2000;20(1):28-57.

75. MacKenzie B, Korfei M, Henneke I, Sibinska Z, Tian X, Hezel S, Dilai S, Wasnick R, Schneider B, Wilhelm J, El Agha E, Klepetko W, Seeger W, Schermuly R, Gunther A, Bellusci S. Increased FGF1FGFRc expression in idiopathic pulmonary fibrosis. Respir Res 2015;16:83.

76. Paez-Ribes M, Allen E, Hudock J, Takeda T, Okuyama H, Vinals F, Inoue M, Bergers G, Hanahan D, Casanovas $O$. Antiangiogenic therapy elicits malignant progression of tumors to increased local invasion and distant metastasis. Cancer Cell 2009;15(3):220-231.

77. Ebos JM, Lee CR, Cruz-Munoz W, Bjarnason GA, Christensen JG, Kerbel RS. Accelerated metastasis after short-term treatment with a potent inhibitor of tumor angiogenesis. Cancer Cell 2009;15(3):232239.

78. Sennino B, McDonald DM. Controlling escape from angiogenesis inhibitors. Nat Rev Cancer 2012;12(10):699-709.

79. Small D. FLT3 mutations: biology and treatment. Hematol-Am Soc Hematol Educ Program 2006:178184.

80. Zorn JA, Wang Q, Fujimura E, Barros T, Kuriyan J. Crystal structure of the FLT3 kinase domain bound to the inhibitor Quizartinib (AC220). PLoS ONE 2015;10(4):e0121177.

81. Zarrinkar PP, Gunawardane RN, Cramer MD, Gardner MF, Brigham D, Belli B, Karaman MW, Pratz KW, Pallares G, Chao Q, Sprankle KG, Patel HK, Levis M, Armstrong RC, James J, Bhagwat SS. AC220 is a uniquely potent and selective inhibitor of FLT3 for the treatment of acute myeloid leukemia (AML). Blood 2009;114(Copyright (C) 2011 American Chemical Society (ACS). All Rights Reserved.):29842992.

82. Smith CC, Lasater EA, Lin KC, Wang Q, McCreery MQ, Stewart WK, Damon LE, Perl AE, Jeschke GR, Sugita M, Carroll M, Kogan SC, Kuriyan J, Shah NP. Crenolanib is a selective type I pan-FLT3 inhibitor. Proc Natl Acad Sci USA 2014;111(14):5319-5324.

83. Zhao Z, Wu H, Wang L, Liu Y, Knapp S, Liu Q, Gray NS. Exploration of Type II Binding Mode: A Privileged Approach for Kinase Inhibitor Focused Drug Discovery? ACS Chem Biol 2014;9(6):12301241.

84. Lennartsson J, Ronnstrand L. Stem cell factor receptor/c-Kit: from basic science to clinical implications. Physiol Rev 2012;92(4):1619-1649.

85. Nishida T, Doi T, Naito Y. Tyrosine kinase inhibitors in the treatment of unresectable or metastatic gastrointestinal stromal tumors. Expert Opin Pharmacother 2014;15(14):1979-1989.

86. Ustun C, Corless CL, Savage N, Fiskus W, Manaloor E, Heinrich MC, Lewis G, Ramalingam P, Kepten I, Jillella A, Bhalla K. Chemotherapy and dasatinib induce long-term hematologic and molecular remission in systemic mastocytosis with acute myeloid leukemia with KIT D816V. Leuk Res 2009;33(5):735-741.

87. Zhu Y, Knolhoff BL, Meyer MA, Nywening TM, West BL, Luo J, Wang-Gillam A, Goedegebuure SP, Linehan DC, DeNardo DG. CSF1/CSF1R Blockade Reprograms Tumor-Infiltrating Macrophages and Improves Response to T Cell Checkpoint Immunotherapy in Pancreatic Cancer Models. Cancer Res 2014;74(18):5057-5069.

88. Hallinan N, Finn S, Cuffe S, Rafee S, O'Byrne K, Gately K. Targeting the fibroblast growth factor receptor family in cancer. Cancer Treat Rev 2016;46:51-62.

89. Sohl CD, Ryan MR, Luo B, Frey KM, Anderson KS. Illuminating the molecular mechanisms of tyrosine kinase inhibitor resistance for the FGFR1 gatekeeper mutation: the Achilles' heel of targeted therapy. ACS Chem Biol 2015;10(5):1319-1329.

90. Bean J, Brennan C, Shih JY, Riely G, Viale A, Wang L, Chitale D, Motoi N, Szoke J, Broderick S, Balak M, Chang WC, Yu CJ, Gazdar A, Pass H, Rusch V, Gerald W, Huang SF, Yang PC, Miller V, Ladanyi M, Yang $\mathrm{CH}$, Pao W. MET amplification occurs with or without T790M mutations in EGFR mutant lung tumors with acquired resistance to gefitinib or erlotinib. Proc Natl Acad Sci USA 2007;104(52):2093220937.

91. Di Renzo MF, Olivero M, Giacomini A, Porte H, Chastre E, Mirossay L, Nordlinger B, Bretti S, Bottardi S, Giordano $S$, et al. Overexpression and amplification of the met/HGF receptor gene during the progression of colorectal cancer. Clin Cancer Res 1995;1(2):147-154.

92. Yakes FM, Chen J, Tan J, Yamaguchi K, Shi Y, Yu P, Qian F, Chu F, Bentzien F, Cancilla B, Orf J, You A, Laird AD, Engst S, Lee L, Lesch J, Chou Y-C, Joly AH. Cabozantinib (XL184), a Novel MET and VEGFR2 Inhibitor, Simultaneously Suppresses Metastasis, Angiogenesis, and Tumor Growth. Mol Cancer Ther $2011 ; 10(12): 2298-2308$.

93. Peters S, Adjei AA. MET: a promising anticancer therapeutic target. Nat Rev Clin Oncol 2012;9(6):314326. 
94. Duchemann B, Friboulet $L$, Besse B. Therapeutic management of ALK+ nonsmall cell lung cancer patients. Eur Respir J 2015;46(1):230-242.

95. Gambacorti Passerini C, Farina F, Stasia A, Redaelli S, Ceccon M, Mologni L, Messa C, Guerra L, Giudici G, Sala E, Mussolin L, Deeren D, King MH, Steurer M, Ordemann R, Cohen AM, Grube M, Bernard L, Chiriano G, Antolini L, Piazza R. Crizotinib in advanced, chemoresistant anaplastic lymphoma kinasepositive lymphoma patients. J Natl Cancer Inst 2014;106(2):djt378.

96. Choi YL, Soda M, Yamashita Y, Ueno T, Takashima J, Nakajima T, Yatabe Y, Takeuchi K, Hamada T, Haruta $H$, Ishikawa $Y$, Kimura $H$, Mitsudomi T, Tanio $Y$, Mano H, Grp ALCS. EML4-ALK Mutations in Lung Cancer That Confer Resistance to ALK Inhibitors. New Engl J Med 2010;363(18):1734-1739.

97. Fontana D, Ceccon M, Gambacorti-Passerini C, Mologni L. Activity of second-generation ALK inhibitors against crizotinib-resistant mutants in an NPM-ALK model compared to EML4-ALK. Cancer Med 2015;4(7):953-965.

98. Zhang I, Zaorsky NG, Palmer JD, Mehra R, Lu B. Targeting brain metastases in ALK-rearranged nonsmall-cell lung cancer. Lancet Oncol 2015;16(13):e510-e521.

99. Johnson TW, Richardson PF, Bailey S, Brooun A, Burke BJ, Collins MR, Cui JJ, Deal JG, Deng Y-L, Dinh D, Engstrom LD, He M, Hoffman J, Hoffman RL, Huang Q, Kania RS, Kath JC, Lam H, Lam JL, Le PT, Lingardo L, Liu W, McTigue M, Palmer CL, Sach NW, Smeal T, Smith GL, Stewart AE, Timofeevski S, Zhu H, Zhu J, Zou HY, Edwards MP. Discovery of (10R)-7-Amino-12-fluoro-2,10,16-trimethyl-15-oxo$10,15,16,17$-tetrahydro-2H-8,4-(metheno)pyrazolo[4,3-h][2,5,11]-benzoxadiazacyclotetradecine-3carbonitrile (PF-06463922), a Macrocyclic Inhibitor of Anaplastic Lymphoma Kinase (ALK) and c-ros Oncogene 1 (ROS1) with Preclinical Brain Exposure and Broad-Spectrum Potency against ALKResistant Mutations. J Med Chem 2014;57(11):4720-4744.

100. Ryan PD, Goss PE. The emerging role of the insulin-like growth factor pathway as a therapeutic target in cancer. Oncologist 2008;13(1):16-24.

101. Pillai RN, Ramalingam SS. Inhibition of insulin-like growth factor receptor: end of a targeted therapy? Transl Lung Cancer Res 2012;2(1):14-22.

102. Gombos A, Metzger-Filho O, Dal LL, Awada-Hussein A. Clinical development of insulin-like growth factor receptor-1 (IGF-1R) inhibitors: At the crossroad? Invest New Drugs 2012;30(6):2433-2442.

103. Vaishnavi A, Le AT, Doebele RC. TRKing down an old oncogene in a new era of targeted therapy. Cancer Discov 2015;5(1):25-34.

104. Thiele CJ, Li Z, McKee AE. On Trk-The TrkB Signal Transduction Pathway Is an Increasingly Important Target in Cancer Biology. Clin Cancer Res 2009;15(19):5962-5967.

105. Ardini E, Menichincheri M, Banfi P, Bosotti R, De Ponti C, Pulci R, Ballinari D, Ciomei M, Texido G, Degrassi A, Avanzi N, Amboldi N, Saccardo MB, Casero D, Orsini P, Bandiera T, Mologni L, Anderson D, Wei G, Harris J, Vernier J-M, Li G, Felder E, Donati D, Isacchi A, Pesenti E, Magnaghi P, Galvani A. Entrectinib, a Pan-TRK, ROS1, and ALK Inhibitor with Activity in Multiple Molecularly Defined Cancer Indications. Mol Cancer Ther 2016;15(4):628-639.

106. Graham DK, DeRyckere D, Davies KD, Earp HS. The TAM family: phosphatidylserine-sensing receptor tyrosine kinases gone awry in cancer. Nat Rev Cancer 2014;14(12):769-785.

107. Myers SH, Brunton VG, Unciti-Broceta A. AXL Inhibitors in Cancer: A Medicinal Chemistry Perspective. J Med Chem 2016;59(8):3593-3608.

108. Mori M, Kaneko N, Ueno Y, Tanaka R, Cho K, Saito R, Kondoh Y, Shimada I, Kuromitsu S. ASP2215, a novel FLT3/AXL inhibitor: Preclinical evaluation in acute myeloid leukemia (AML). J Clin Oncol 2014;32 (Suppl. 5):Abs. 7070.

109. Mulligan LM. RET revisited: expanding the oncogenic portfolio. Nat Rev Cancer 2014;14(3):173-186.

110. Drilon A, Wang L, Hasanovic A, Suehara Y, Lipson D, Stephens P, Ross J, Miller V, Ginsberg M, Zakowski MF, Kris MG, Ladanyi M, Rizvi N. Response to Cabozantinib in patients with RET fusionpositive lung adenocarcinomas. Cancer Discov 2013;3(6):630-635.

111. Gautschi O, Zander T, Keller FA, Strobel K, Hirschmann A, Aebi S, Diebold J. A patient with lung adenocarcinoma and RET fusion treated with vandetanib. J Thorac Oncol 2013;8(5):e43-44.

112. Krajewska J, Kukulska A, Jarzab B. Efficacy of lenvatinib in treating thyroid cancer. Expert Opin Pharmacother 2016;17(12):1683-1691.

113. Krajewska J, Olczyk T, Jarzab B. Cabozantinib for the treatment of progressive metastatic medullary thyroid cancer. Expert Rev Clin Pharmacol 2016;9(1):69-79.

114. Hayman SR, Leung N, Grande JP, Garovic VD. VEGF inhibition, hypertension, and renal toxicity. Curr Oncol Rep 2012;14(4):285-294.

115. Jordan AM, Begum H, Fairweather E, Fritzl S, Goldberg K, Hopkins GV, Hamilton NM, Lyons AJ, March HN, Newton R, Small HF, Vishwanath S, Waddell ID, Waszkowycz B, Watson AJ, Ogilvie DJ. Anilinoquinazoline inhibitors of the RET kinase domain-Elaboration of the 7-position. Bioorg Med Chem Lett 2016;26(11):2724-2729.

116. Song M. Progress in Discovery of KIF5B-RET Kinase Inhibitors for the Treatment of Non-Small-Cell Lung Cancer. J Med Chem 2015;58(9):3672-3681.

117. Drabsch $Y$, Dijke P. TGF- $\beta$ signalling and its role in cancer progression and metastasis. Cancer Metast Rev 2012;31(3-4):553-568.

118. Zhou L, McMahon C, Bhagat T, Alencar C, Yu Y, Fazzari M, Sohal D, Heuck C, Gundabolu K, Ng C, Mo Y, Shen W, Wickrema A, Kong G, Friedman E, Sokol L, Mantzaris G, Pellagatti A, Boultwood J, Platanias LC, Steidl U, Yan L, Yingling JM, Lahn MM, List A, Bitzer M, Verma A. Reduced SMAD7 Leads to Overactivation of TGF-beta Signaling in MDS that Can Be Reversed by a Specific Inhibitor of TGFbeta Receptor I Kinase. Cancer Res 2011;71(3):955-963.

119. Akhurst RJ, Hata A. Targeting the TGF[beta] signalling pathway in disease. Nat Rev Drug Discov 2012;11(10):790-811. 
120. Quintas-Cardama A, Kantarjian $\mathrm{H}$, Cortes J, Verstovsek S. Janus kinase inhibitors for the treatment of myeloproliferative neoplasias and beyond. Nat Rev Drug Discov 2011;10(2):127-140.

121. Quintas-Cardama A, Vaddi K, Liu P, Manshouri T, Li J, Scherle PA, Caulder E, Wen X, Li Y, Waeltz P, Rupar M, Burn T, Lo Y, Kelley J, Covington M, Shepard S, Rodgers JD, Haley P, Kantarjian H, Fridman JS, Verstovsek S. Preclinical characterization of the selective JAK1/2 inhibitor INCB018424: therapeutic implications for the treatment of myeloproliferative neoplasms. Blood 2010;115(15):3109-3117.

122. Mascarenhas J, Hoffman R. A comprehensive review and analysis of the effect of ruxolitinib therapy on the survival of patients with myelofibrosis. Blood 2013;121(24):4832-4837.

123. Kalota A, Jeschke GR, Carroll M, Hexner EO. Intrinsic Resistance to JAK2 Inhibition in Myelofibrosis. Clin Cancer Res 2013;19(7):1729-1739.

124. Sonbol MB, Firwana B, Zarzour A, Morad M, Rana V, Tiu RV. Comprehensive review of JAK inhibitors in myeloproliferative neoplasms. Ther Adv Hematol 2013;4(1):15-35.

125. Alinari L, Quinion C, Blum KA. Bruton's tyrosine kinase inhibitors in B-cell non-Hodgkin's lymphomas. Clin Pharmacol Ther 2015;97(5):469-477.

126. Kawakami Y, Hartman SE, Kinoshita E, Suzuki H, Kitaura J, Yao L, Inagaki N, Franco A, Hata D, Maeda-Yamamoto M, Fukamachi H, Nagai H, Kawakami T. Terreic acid, a quinone epoxide inhibitor of Bruton's tyrosine kinase. Proc Natl Acad Sci USA 1999;96(5):2227-2232.

127. Mahajan S, Ghosh S, Sudbeck EA, Zheng Y, Downs S, Hupke M, Uckun FM. Rational design and synthesis of a novel anti-leukemic agent targeting Bruton's tyrosine kinase (BTK), LFM-A13 [alphacyano-beta-hydroxy-beta-methyl-N-(2, 5-dibromophenyl)propenamide]. J Biol Chem 1999;274(14):9587-9599.

128. Pan Z, Scheerens H, Li S-J, Schultz BE, Sprengeler PA, Burrill LC, Mendonca RV, Sweeney MD, Scott KCK, Grothaus PG, Jeffery DA, Spoerke JM, Honigberg LA, Young PR, Dalrymple SA, Palmer JT. Discovery of selective irreversible inhibitors for bruton's tyrosine kinase. ChemMedChem 2007;2(1):58-61.

129. Honigberg LA, Smith AM, Sirisawad M, Verner E, Loury D, Chang B, Li S, Pan Z, Thamm DH, Miller RA, Buggy JJ. The Bruton tyrosine kinase inhibitor PCI-32765 blocks B-cell activation and is efficacious in models of autoimmune disease and B-cell malignancy. Proc Natl Adad Sci USA 2010;107(29):1307513080, S13075/13071-S13075/13073.

130. Lee BY, Timpson P, Horvath LG, Daly RJ. FAK signaling in human cancer as a target for therapeutics. Pharmacol Ther 2015;146:132-149.

131. Yoon H, Dehart JP, Murphy JM, Lim S-TS. Understanding the Roles of FAK in Cancer: Inhibitors, Genetic Models, and New Insights. J Histochem and Cytochem 2015;63(2):114-128.

132. Sulzmaier FJ, Jean C, Schlaepfer DD. FAK in cancer: mechanistic findings and clinical applications. Nat Rev Cancer 2014;14(9):598-610.

133. Iwatani M, Iwata H, Okabe A, Skene RJ, Tomita N, Hayashi Y, Aramaki Y, Hosfield DJ, Hori A, Baba A, Miki H. Discovery and characterization of novel allosteric FAK inhibitors. Eur J Med Chem 2013;61(0):49-60.

134. Geahlen RL. Getting Syk: spleen tyrosine kinase as a therapeutic target. Trends Pharmacol Sci 2014;35(8):414-422.

135. Seda V, Mraz M. B-cell receptor signalling and its crosstalk with other pathways in normal and malignant cells. Eur J Haematol 2015;94(3):193-205.

136. Lucas MC, Tan S-L. Small-molecule inhibitors of spleen tyrosine kinase as therapeutic agents for immune disorders: will promise meet expectations? Future Med Chem 2014;6(16):1811-1827.

137. McAdoo SP, Tam FWK, Pandian R, Bolos J, Castaner R. Fostamatinib disodium: tyrosine-protein kinase SYK/FLT3 inhibitor treatment of rheumatoid arthritis oncolytic. Drugs Future 2011;36(4):273-280.

138. Coffey G, Betz A, DeGuzman F, Pak Y, Inagaki M, Baker DC, Hollenbach SJ, Pandey A, Sinha U. The novel kinase inhibitor PRT062070 (Cerdulatinib) demonstrates efficacy in models of autoimmunity and B-cell cancer. J Pharmacol Exp Ther 2014;351(3):538-548, 511 pp.

139. Bjornsti M-A, Houghton PJ. Lost in translation: Dysregulation of cap-dependent translation and cancer. Cancer Cell 2004;5(6):519-523.

140. Faivre S, Kroemer G, Raymond E. Current development of mTOR inhibitors as anticancer agents. Nat Rev Drug Discov 2006;5(8):671-688.

141. Thomas GV, Tran C, Mellinghoff IK, Welsbie DS, Chan E, Fueger B, Czernin J, Sawyers CL. Hypoxiainducible factor determines sensitivity to inhibitors of mTOR in kidney cancer. Nat Med $2006 ; 12(1): 122-127$.

142. Bjornsti MA, Houghton PJ. The TOR pathway: a target for cancer therapy. Nat Rev Cancer 2004;4(5):335-348.

143. Raimondo L, D'Amato V, Servetto A, Rosa R, Marciano R, Formisano L, Di Mauro C, Clara Orsini R, Cascetta P, Ciciola P, De Maio AP, Di Renzo MF, Cosconati S, Bruno A, Randazzo A, Napolitano F, Montuori N, Veneziani BM, De Placido S, Bianco R. Everolimus induces Met inactivation by disrupting the FKBP12/Met complex. Oncotarget 2016;7(26):40073-40084.

144. Grant S. Cotargeting survival signaling pathways in cancer. J Clin Invest 2008;118(9):3003-3006.

145. Workman P, Clarke PA, Raynaud FI, van Montfort RL. Drugging the PI3 kinome: from chemical tools to drugs in the clinic. Cancer Res 2010;70(6):2146-2157.

146. Thorpe LM, Yuzugullu H, Zhao JJ. PI3K in cancer: divergent roles of isoforms, modes of activation and therapeutic targeting. Nat Rev Cancer 2015;15(1):7-24.

147. Workman P, van MRLM. Unveiling the secrets of the ancestral PI3 kinase Vps34. Cancer Cell 2010;17(Copyright (C) 2012 American Chemical Society (ACS). All Rights Reserved.):421-423.

148. Ikeda $H$, Hideshima $T$, Fulciniti $M$, Perrone $G$, Miura N, Yasui $H$, Okawa $Y$, Kiziltepe $T$, Santo L, Vallet $S$, Cristea D, Calabrese E, Gorgun G, Raje NS, Richardson P, Munshi NC, Lannutti BJ, Puri KD, Giese NA, 
Anderson KC. PI3K/p110delta is a novel therapeutic target in multiple myeloma. Blood $2010 ; 116(9): 1460-1468$.

149. Ali K, Soond DR, Pineiro R, Hagemann T, Pearce W, Lim EL, Bouabe H, Scudamore CL, Hancox T, Maecker H, Friedman L, Turner M, Okkenhaug K, Vanhaesebroeck B. Inactivation of PI(3)K p110[dgr] breaks regulatory T-cell-mediated immune tolerance to cancer. Nature 2014;510(7505):407-411.

150. Utermark T, Rao T, Cheng H, Wang Q, Lee SH, Wang ZC, Iglehart JD, Roberts TM, Muller WJ, Zhao JJ. The p110alpha and p110beta isoforms of PI3K play divergent roles in mammary gland development and tumorigenesis. Genes Dev 2012;26(14):1573-1586.

151. Busaidy NL, Farooki A, Dowlati A, Perentesis JP, Dancey JE, Doyle LA, Brell JM, Siu LL. Management of metabolic effects associated with anticancer agents targeting the PI3K-Akt-mTOR pathway. J Clin Oncol 2012;30(23):2919-2928.

152. Jarvis LM. PI3K at the clinicl crossroads. Chem Eng News 2011;April 11, 2011:15-19.

153. Collins I. Targeted small-molecule inhibitors of protein kinase B as anticancer agents. Anti-Cancer Agents Med Chem 2009;9(1):32-50.

154. Kondapaka SB, Singh SS, Dasmahapatra GP, Sausville EA, Roy KK. Perifosine, a novel alkylphospholipid, inhibits protein kinase B activation. Mol Cancer Ther 2003;2(11):1093-1103.

155. Cho DC, Figlin RA, Flaherty KT, Michaelson D, Sosman JA, Ghebremichael M, Bowers ME, Mier JW, Atkins MB, McDermott DF. A phase II trial of perifosine in patients with advanced renal cell carcinoma (RCC) who have failed tyrosine kinase inhibitors (TKI). J Clin Oncol 2009;27:15s:Abs. 5101.

156. Barnett SF, Defeo-Jones D, Fu S, Hancock PJ, Haskell KM, Jones RE, Kahana JA, Kral AM, Leander K, Lee LL, Malinowski J, McAvoy EM, Nahas DD, Robinson RG, Huber HE. Identification and characterization of pleckstrin-homology-domain-dependent and isoenzyme-specific Akt inhibitors. Biochem J 2005;385(2):399-408.

157. Wu W-I, Voegtli WC, Sturgis HL, Dizon FP, Vigers GPA, Brandhuber BJ. Crystal Structure of Human AKT1 with an Allosteric Inhibitor Reveals a New Mode of Kinase Inhibition. PLoS ONE 2010;5(9):e12913.

158. Ashwell MA, Lapierre JM, Brassard C, Bresciano K, Bull C, Cornell-Kennon S, Eathiraj S, France DS, Hall T, Hill J, Kelleher E, Khanapurkar S, Kizer D, Koerner S, Link J, Liu Y, Makhija S, Moussa M, Namdev N, Nguyen K, Nicewonger R, Palma R, Szwaya J, Tandon M, Uppalapati U, Vensel D, Volak LP, Volckova E, Westlund N, Wu H, Yang RY, Chan TC. Discovery and optimization of a series of 3-(3phenyl-3H-imidazo[4,5-b]pyridin-2-yl)pyridin-2-amines: orally bioavailable, selective, and potent ATPindependent Akt inhibitors. J Med Chem 2012;55(11):5291-5310.

159. Peifer C, Alessi DR. Small-molecule inhibitors of PDK1. ChemMedChem 2008;3(12):1810-1838.

160. Graff JR, McNulty AM, Hanna KR, Konicek BW, Lynch RL, Bailey SN, Banks C, Capen A, Goode R, Lewis JE, Sams L, Huss KL, Campbell RM, Iversen PW, Neubauer BL, Brown TJ, Musib L, Geeganage S, Thornton D. The protein kinase Cbeta-selective inhibitor, Enzastaurin (LY317615. $\mathrm{HCl})$, suppresses signaling through the AKT pathway, induces apoptosis, and suppresses growth of human colon cancer and glioblastoma xenografts. Cancer Res 2005;65(16):7462-7469.

161. Raab MS, Breitkreutz I, Tonon G, Zhang J, Hayden PJ, Nguyen T, Fruehauf JH, Lin BK, Chauhan D, Hideshima T, Munshi NC, Anderson KC, Podar K. Targeting PKC: a novel role for beta-catenin in ER stress and apoptotic signaling. Blood 2009;113(7):1513-1521.

162. Crump M, Leppä S, Fayad L, Lee JJ, Di Rocco A, Ogura M, Hagberg H, Schnell F, Rifkin R, Mackensen A, Offner F, Pinter-Brown L, Smith S, Tobinai K, Yeh S-P, Hsi ED, Nguyen T, Shi P, Hahka-Kemppinen M, Thornton D, Lin B, Kahl B, Schmitz N, Savage KJ, Habermann T. Randomized, Double-Blind, Phase III Trial of Enzastaurin Versus Placebo in Patients Achieving Remission After First-Line Therapy for High-Risk Diffuse Large B-Cell Lymphoma. J Clin Oncol 2016.

163. Tse AN, Carvajal R, Schwartz GK. Targeting Checkpoint Kinase 1 in Cancer Therapeutics. Clin Cancer Res 2007;13(7):1955-1960.

164. Barry EV, Clark JJ, Cools J, Roesel J, Gilliland DG. Uniform sensitivity of FLT3 activation loop mutants to the tyrosine kinase inhibitor midostaurin. Blood 2007;110(13):4476-4479.

165. Roffey J, Rosse C, Linch M, Hibbert A, McDonald NQ, Parker PJ. Protein kinase C intervention - the state of play. Curr Opin Cell Biol 2009;21(2):268-279.

166. Arlauckas SP, Popov AV, Delikatny EJ. Choline kinase alpha-Putting the ChoK-hold on tumor metabolism. Prog Lipid Res 2016: Ahead of Print.

167. Asim M, Massie CE, Neal DE. Kinase joins the chaperone club: Androgen-regulated kinome reveals choline kinase alpha as a potential drug target in prostate cancer. Mol Cell Oncol 2016: Ahead of Print.

168. Challapalli A, Trousil S, Hazell S, Kozlowski K, Gudi M, Aboagye EO, Mangar S. Exploiting altered patterns of choline kinase-alpha expression on human prostate tissue to prognosticate prostate cancer. J Clin Pathol 2015;68(9):703-709.

169. Hong BS, Allali-Hassani A, Tempel W, Finerty PJ, Jr., MacKenzie F, Dimov S, Vedadi M, Park H-W. Crystal Structures of Human Choline Kinase Isoforms in Complex with Hemicholinium-3: Single Amino Acid Near the Active Site Influences Inhibitor Sensitivity. J Biol Chem 2010;285(21):16330-16340.

170. Cozza G, Moro S. Casein kinase 2 (CK2) inhibitors: emerging anticancer therapeutic agents? In: Prudhomme M, editor. Advances in Anticancer Agents in Medicinal Chemistry. Volume 1: Bentham Science Publishers Ltd.; 2013. pp 3-42.

171. Mandato E, Manni S, Zaffino F, Semenzato G, Piazza F. Targeting CK2-driven non-oncogene addiction in B-cell tumors. Oncogene 2016:Ahead of Print.

172. Slaton JW, Unger GM, Sloper DT, Davis AT, Ahmed K. Induction of Apoptosis by Antisense CK2 in Human Prostate Cancer Xenograft Model. Mol Cancer Res 2004;2(12):712-721.

173. Pierre F, Chua PC, O'Brien SE, Siddiqui-Jain A, Bourbon P, Haddach M, Michaux J, Nagasawa J, Schwaebe MK, Stefan E, Vialettes A, Whitten JP, Chen TK, Darjania L, Stansfield R, Anderes K, Bliesath J, Drygin D, Ho C, Omori M, Proffitt C, Streiner N, Trent K, Rice WG, Ryckman DM. Discovery and SAR 
of 5-(3-Chlorophenylamino)benzo[c][2,6]naphthyridine-8-carboxylic Acid (CX-4945), the first clinical stage inhibitor of protein kinase CK2 for the treatment of cancer. J Med Chem 2011;54(2):635-654.

174. Cho Y-S, Kang Y, Kim K, Cha Y-j, Cho H-S. The crystal structure of MPK38 in complex with OTSSP167, an orally administrative MELK selective inhibitor. Biochem Biophys Res Commun 2014;447(1):7-11.

175. Mondello P, Cuzzocrea S, Mian M. Pim kinases in hematological malignancies: where are we now and where are we going? J Hematol Oncol 2014;7:95/91-95/99.

176. Keane NA, Reidy M, Natoni A, Raab MS, O'Dwyer M. Targeting the Pim kinases in multiple myeloma. Blood Cancer Journal 2015;5:e325.

177. Le BT, Kumarasiri M, Adams JRJ, Yu M, Milne R, Sykes MJ, Wang S. Targeting Pim kinases for cancer treatment: opportunities and challenges. Future Med Chem 2015;7(1):35-53.

178. Sullivan RJ, Flaherty K. MAP kinase signaling and inhibition in melanoma. Oncogene 2013;32(19):2373-2379.

179. Tsai J, Lee JT, Wang W, Zhang J, Cho H, Mamo S, Bremer R, Gillette S, Kong J, Haass NK, Sproesser K, Li L, Smalley KSM, Fong D, Zhu Y-L, Marimuthu A, Nguyen H, Lam B, Liu J, Cheung I, Rice J, Suzuki Y, Luu C, Settachatgul C, Shellooe R, Cantwell J, Kim S-H, Schlessinger J, Zhang KYJ, West BL, Powell B, Habets G, Zhang C, Ibrahim PN, Hirth P, Artis DR, Herlyn M, Bollag G. Discovery of a selective inhibitor of oncogenic B-Raf kinase with potent antimelanoma activity. Proc Natl Acad Sci USA 2008;105(8):3041-3046.

180. Rheault TR, Stellwagen JC, Adjabeng GM, Hornberger KR, Petrov KG, Waterson AG, Dickerson SH, Mook RA, Laquerre SG, King AJ, Rossanese OW, Arnone MR, Smitheman KN, Kane-Carson LS, Han C, Moorthy GS, Moss KG, Uehling DE. Discovery of Dabrafenib: A Selective Inhibitor of Raf Kinases with Antitumor Activity against B-Raf-Driven Tumors. ACS Med Chem Lett 2013;4(3):358-362.

181. Hatzivassiliou G, Song K, Yen I, Brandhuber BJ, Anderson DJ, Alvarado R, Ludlam MJ, Stokoe D, Gloor SL, Vigers G, Morales T, Aliagas I, Liu B, Sideris S, Hoeflich KP, Jaiswal BS, Seshagiri S, Koeppen H, Belvin M, Friedman LS, Malek S. RAF inhibitors prime wild-type RAF to activate the MAPK pathway and enhance growth. Nature 2010;464(7287):431-435.

182. Heidorn SJ, Milagre C, Whittaker S, Nourry A, Niculescu-Duvas I, Dhomen N, Hussain J, Reis-Filho JS, Springer CJ, Pritchard C, Marais R. Kinase-dead BRAF and oncogenic RAS cooperate to drive tumor progression through CRAF. Cell 2010;140(Copyright (C) 2011 American Chemical Society (ACS). All Rights Reserved.):209-221.

183. Anforth R, Fernandez-Penas P, Long GV. Cutaneous toxicities of RAF inhibitors. Lancet Oncol 2013;14(1):e11-e18.

184. Uehling DE, Harris PA. Recent progress on MAP kinase pathway inhibitors. Bioorg Med Chem Lett 2015;25(19):4047-4056.

185. Davies H, Bignell GR, Cox C, Stephens P, Edkins S, Clegg S, Teague J, Woffendin H, Garnett MJ, Bottomley W, Davis N, Dicks E, Ewing R, Floyd Y, Gray K, Hall S, Hawes R, Hughes J, Kosmidou V, Menzies A, Mould C, Parker A, Stevens C, Watt S, Hooper S, Wilson R, Jayatilake H, Gusterson BA, Cooper C, Shipley J, Hargrave D, Pritchard-Jones K, Maitland N, Chenevix-Trench G, Riggins GJ, Bigner DD, Palmieri G, Cossu A, Flanagan A, Nicholson A, Ho JWC, Leung SY, Yuen ST, Weber BL, Seigler HF, Darrow TL, Paterson $H$, Marais R, Marshall CJ, Wooster R, Stratton MR, Futreal PA. Mutations of the BRAF gene in human cancer. Nature 2002;417(6892):949-954.

186. Jang S, Atkins MB. Which drug, and when, for patients with BRAF-mutant melanoma? Lancet Oncol 2013;14(2):e60-e69.

187. Larkin J, Ascierto PA, Dréno B, Atkinson V, Liszkay G, Maio M, Mandalà M, Demidov L, Stroyakovskiy D, Thomas L, de la Cruz-Merino L, Dutriaux C, Garbe C, Sovak MA, Chang I, Choong N, Hack SP, McArthur GA, Ribas A. Combined Vemurafenib and Cobimetinib in BRAF-Mutated Melanoma. New Engl J Med 2014;371(20):1867-1876.

188. Mandal R, Becker S, Strebhardt K. Stamping out RAF and MEK1/2 to inhibit the ERK1/2 pathway: an emerging threat to anticancer therapy. Oncogene 2016;35(20):2547-2561.

189. Young $\mathrm{K}$, Minchom A, Larkin J. BRIM-1, -2 and -3 trials: improved survival with vemurafenib in metastatic melanoma patients with a BRAF(V600E) mutation. Future Oncol 2012;8(5):499-507.

190. Britten CD. PI3K and MEK inhibitor combinations: examining the evidence in selected tumor types. Cancer Chemother Pharmacol 2013;71(6):1395-1409.

191. Samatar AA, Poulikakos PI. Targeting RAS-ERK signalling in cancer: promises and challenges. Nat Rev Drug Discov 2014;13(12):928-942.

192. Hatzivassiliou G, Liu B, O'Brien C, Spoerke JM, Hoeflich KP, Haverty PM, Soriano R, Forrest WF, Heldens S, Chen H, Toy K, Ha C, Zhou W, Song K, Friedman LS, Amler LC, Hampton GM, Moffat J, Belvin M, Lackner MR. ERK Inhibition Overcomes Acquired Resistance to MEK Inhibitors. Mol Cancer Ther 2012;11(5):1143-1154.

193. Trempolec N, Dave-Coll N, Nebreda AR. SnapShot: p38 MAPK Signaling. Cell 2013;152(3):656656.e651.

194. Goldstein DM, Kuglstatter A, Lou Y, Soth MJ. Selective p38a Inhibitors Clinically Evaluated for the Treatment of Chronic Inflammatory Disorders. J Med Chem 2010;53(6):2345-2353.

195. Martin ED, Bassi R, Marber MS. p38 MAPK in cardioprotection - are we there yet? Br J Pharmacol 2015;172(8):2101-2113.

196. Wagner EF, Nebreda AR. Signal integration by JNK and p38 MAPK pathways in cancer development. Nat Rev Cancer 2009;9(8):537-549.

197. Hou JQ, Lam F, Proud C, Wang SD. Targeting Mnks for Cancer Therapy. Oncotarget 2012;3(2):118131.

198. Diab S, Li P, Basnet SKC, Lu JF, Yu MF, Albrecht H, Milne RW, Wang SD. Unveiling new chemical scaffolds as Mnk inhibitors. Future Med Chem 2016;8(3):271-285. 
199. Teo T, Lam F, Yu MF, Yang YC, Basnet SKC, Albrecht H, Sykes MJ, Wang SD. Pharmacologic Inhibition of MNKs in Acute Myeloid Leukemia. Mol Pharmacol 2015;88(2):380-389.

200. Lim S, Kaldis P. Cdks, cyclins and CKIs: roles beyond cell cycle regulation. Development 2013;140(15):3079-3093.

201. Asghar U, Witkiewicz AK, Turner NC, Knudsen ES. The history and future of targeting cyclin-dependent kinases in cancer therapy. Nat Rev Drug Discov 2015;14(2):130-146.

202. Wang S, Fischer PM. Cyclin-dependent kinase 9: a key transcriptional regulator and potential drug target in oncology, virology and cardiology. Trends Pharmacol Sci 2008;29(6):302-313.

203. Lücking U. Sulfoximines: A Neglected Opportunity in Medicinal Chemistry. Angew Chem Int Ed 2013;52(36):9399-9408.

204. Kollareddy M, Zheleva D, Dzubak P, Brahmkshatriya PS, Lepsik M, Hajduch M. Aurora kinase inhibitors: Progress towards the clinic. Invest New Drugs 2012;30(6):2411-2432.

205. Bavetsias V, Linardopoulos S. Aurora kinase inhibitors: current status and outlook. Frontiers in Oncology 2015;5.

206. McInnes C, Wyatt MD. PLK1 as an oncology target: current status and future potential. Drug Discov Today $2011 ; 16(13-14): 619-625$

207. Haupenthal J, Bihrer V, Korkusuz H, Kollmar O, Schmithals C, Kriener S, Engels K, Pleli T, Benz A Canamero M, Longerich T, Kronenberger B, Richter S, Waidmann O, Vogl TJ, Zeuzem S, Piiper A. Reduced Efficacy of the PIk1 Inhibitor BI 2536 on the Progression of Hepatocellular Carcinoma due to Low Intratumoral Drug Levels. Neoplasia 2012;14(5):410-+.

208. Luo J, Emanuele MJ, Li D, Creighton CJ, Schlabach MR, Westbrook TF, Wong KK, Elledge SJ. A genome-wide RNAi screen identifies multiple synthetic lethal interactions with the Ras oncogene. Cell 2009;137(5):835-848.

209. Manic G, Obrist F, Sistigu A, Vitale I. Trial Watch: Targeting ATM-CHK2 and ATR-CHK1 pathways for anticancer therapy. Mol Cell Oncol 2016: Ahead of Print.

210. Dai Y, Grant S. New insights into checkpoint kinase 1 in the DNA damage response signaling network. Clin Cancer Res 2010;16(2):376-383.

211. Montagnoli A, Moll J, Colotta F. Targeting cell division cycle 7 kinase: a new approach for cancer therapy. Clin Cancer Res 2010;16(18):4503-4508.

212. Swords R, Mahalingam D, O'Dwyer M, Santocanale C, Kelly K, Carew J, Giles F. Cdc7 kinase: A new target for drug development. Eur J Cancer 2010;46(1):33-40.

213. Lipinski CA. Lead- and drug-like compounds: the rule-of-five revolution. Drug Discovery Today: Technologies 2004;1(4):337 -341.

214. Veber DF, Johnson SR, Cheng H-Y, Smith BR, Ward KW, Kopple KD. Molecular properties that influence the oral bioavailability of drug candidates. J Med Chem 2002;45(12):2615-2623.

215. Lipinski CA. Drug-like properties and the causes of poor solubility and poor permeability. J Pharmacol Toxicol Methods 2000;44(1):235-249.

216. Kumar A, Voet A, Zhang KYJ. Fragment Based Drug Design: From Experimental to Computational Approaches. Curr Med Chem 2012;19(30):5128-5147.

217. Donald A, McHardy T, Rowlands MG, Hunter LJ, Davies TG, Berdini V, Boyle RG, Aherne GW, Garrett MD, Collins I. Rapid evolution of 6-phenylpurine inhibitors of protein kinase B through structure-based design. J Med Chem 2007;50(10):2289-2292.

218. Collins I, Caldwell J, Fonseca T, Donald A, Bavetsias V, Hunter L-JK, Garrett MD, Rowlands MG, Aherne GW, Davies TG, Berdini V, Woodhead SJ, Davis D, Seavers LCA, Wyatt PG, Workman P, McDonald E. Structure-based design of isoquinoline-5-sulfonamide inhibitors of protein kinase B. Bioorg Med Chem 2006;14(4):1255-1273.

219. Caldwell JJ, Davies TG, Donald A, McHardy T, Rowlands MG, Aherne GW, Hunter LK, Taylor K, Ruddle R, Raynaud FI, Verdonk M, Workman P, Garrett MD, Collins I. Identification of 4-(4-aminopiperidin-1yl)-7H-pyrrolo[2,3-d]pyrimidines as selective inhibitors of protein kinase $B$ through fragment elaboration. J Med Chem 2008;51(7):2147-2157.

220. McHardy T, Caldwell JJ, Cheung K-M, Hunter LJ, Taylor K, Rowlands M, Ruddle R, Henley A, Brandon AdH, Valenti M, Davies TG, Fazal L, Seavers L, Raynaud FI, Eccles SA, Aherne GW, Garrett MD, Collins I. Discovery of 4-Amino-1-(7H-pyrrolo[2,3-d]pyrimidin-4-yl)piperidine-4-carboxamides As Selective, Orally Active Inhibitors of Protein Kinase B (Akt). J Med Chem 2010;53(5):2239-2249.

221. Addie $M$, Ballard $P$, Buttar D, Crafter C, Currie G, Davies BR, Debreczeni J, Dry H, Dudley $P$, Greenwood R, Johnson PD, Kettle JG, Lane C, Lamont G, Leach A, Luke RWA, Morris J, Ogilvie D, Page $\mathrm{K}$, Pass M, Pearson S, Ruston L. Discovery of 4-Amino-N-[(1S)-1-(4-chlorophenyl)-3-hydroxypropyl]1-(7H-pyrrolo[2,3-d]pyrimidin-4-yl)piperidine-4-carboxamide (AZD5363), an Orally Bioavailable, Potent Inhibitor of Akt Kinases. J Med Chem 2013;56(5):2059-2073.

222. Nagar B, Bornmann WG, Pellicena P, Schindler T, Veach DR, Miller WT, Clarkson B, Kuriyan J. Crystal Structures of the Kinase Domain of c-Abl in Complex with the Small Molecule Inhibitors PD173955 and Imatinib (STI-571). Cancer Res 2002;62:4236-4243.

223. Xu W, Doshi A, Lei M, Eck MJ, Harrison SC. Crystal structures of c-Src reveal features of its autoinhibitory mechanism. Mol Cell 1999;3(5):629-638.

224. Tokarski JS, Newitt JA, Chang CY, Cheng JD, Wittekind M, Kiefer SE, Kish K, Lee FY, Borzillerri R, Lombardo LJ, Xie D, Zhang Y, Klei HE. The structure of Dasatinib (BMS-354825) bound to activated $A B L$ kinase domain elucidates its inhibitory activity against imatinib-resistant ABL mutants. Cancer Res 2006;66(11):5790-5797.

225. O'Hare T, Shakespeare WC, Zhu X, Eide CA, Rivera VM, Wang F, Adrian LT, Zhou T, Huang W-S, Xu Q, Metcalf CA, III, Tyner JW, Loriaux MM, Corbin AS, Wardwell S, Ning Y, Keats JA, Wang Y, Sundaramoorthi R, Thomas M, Zhou D, Snodgrass J, Commodore L, Sawyer TK, Dalgarno DC, Deininger MWN, Druker BJ, Clackson T. AP24534, a pan-BCR-ABL inhibitor for chronic myeloid 

2009; 16(Copyright (C) 2011 American Chemical Society (ACS). All Rights Reserved.):401-412.
Moy FJ, Lee A, Gavrin LK, Xu Z-B, Sievers A, Kieras E, Stochaj W, Mosyak L, McKew J, Tsao DHH. Novel Synthesis and Structural Characterization of a High-Affinity Paramagnetic Kinase Probe for the Identification of Non-ATP Site Binders by Nuclear Magnetic Resonance. J Med Chem 2010;53(Copyright (C) 2011 American Chemical Society (ACS). All Rights Reserved.):1238-1249.

227. Stamos J, Sliwkowski MX, Eigenbrot C. Structure of the Epidermal Growth Factor Receptor Kinase Domain Alone and in Complex with a 4-Anilinoquinazoline Inhibitor. J Biol Chem 2002;277(48):4626546272.

228. Aertgeerts K, Skene R, Yano J, Sang BC, Zou H, Snell G, Jennings A, Iwamoto K, Habuka N, Hirokawa A, Ishikawa T, Tanaka T, Miki H, Ohta Y, Sogabe S. Structural analysis of the mechanism of inhibition and allosteric activation of the kinase domain of HER2 protein. J Biol Chem 2011;286(21):1875618765.

229. Wood ER, Truesdale AT, McDonald OB, Yuan D, Hassell A, Dickerson SH, Ellis B, Pennisi C, Horne E, Lackey K, Alligood KJ, Rusnak DW, Gilmer TM, Shewchuk L. A Unique Structure for Epidermal Growth Factor Receptor Bound to GW572016 (Lapatinib): Relationships among Protein Conformation, Inhibitor Off-Rate, and Receptor Activity in Tumor Cells. Cancer Res 2004;64(18):6652-6659.

230. Ishikawa T, Seto M, Banno H, Kawakita Y, Oorui M, Taniguchi T, Ohta Y, Tamura T, Nakayama A, Miki $\mathrm{H}$, Kamiguchi H, Tanaka T, Habuka N, Sogabe S, Yano J, Aertgeerts K, Kamiyama K. Design and synthesis of novel human epidermal growth factor receptor 2 (HER2)/epidermal growth factor receptor (EGFR) dual inhibitors bearing a pyrrolo[3,2-d]pyrimidine scaffold. J Med Chem 2011;54(23):80308050.

231. Finlay MRV, Anderton M, Ashton S, Ballard P, Bethel PA, Box MR, Bradbury RH, Brown SJ, Butterworth S, Campbell A, Chorley C, Colclough N, Cross DAE, Currie GS, Grist M, Hassall L, Hill GB, James D, James M, Kemmitt P, Klinowska T, Lamont G, Lamont SG, Martin N, McFarland HL, Mellor MJ, Orme JP, Perkins D, Perkins P, Richmond G, Smith P, Ward RA, Waring MJ, Whittaker D, Wells S, Wrigley GL. Discovery of a Potent and Selective EGFR Inhibitor (AZD9291) of Both Sensitizing and T790M Resistance Mutations That Spares the Wild Type Form of the Receptor. J Med Chem 2014;57(20):8249-8267.

232. Hagel M, Miduturu C, Sheets M, Rubin N, Weng W, Stransky N, Bifulco N, Kim JL, Hodous B, Brooijmans N, Shutes A, Winter C, Lengauer C, Kohl NE, Guzi T. First Selective Small Molecule Inhibitor of FGFR4 for the Treatment of Hepatocellular Carcinomas with an Activated FGFR4 Signaling Pathway. Cancer Discov 2015;5(4):424-437.

233. Tan L, Wang J, Tanizaki J, Huang Z, Aref AR, Rusan M, Zhu SJ, Zhang Y, Ercan D, Liao RG, Capelletti M, Zhou W, Hur W, Kim N, Sim T, Gaudet S, Barbie DA, Yeh JR, Yun CH, Hammerman PS, Mohammadi M, Janne PA, Gray NS. Development of covalent inhibitors that can overcome resistance to firstgeneration FGFR kinase inhibitors. Proc Natl Acad Sci USA 2014;111(45):E4869-4877.

234. Rickert KW, Patel SB, Allison TJ, Byrne NJ, Darke PL, Ford RE, Guerin DJ, Hall DL, Kornienko M, Lu J, Munshi SK, Reid JC, Shipman JM, Stanton EF, Wilson KJ, Young JR, Soisson SM, Lumb KJ. Structural basis for selective small molecule kinase inhibition of activated c-Met. J Biol Chem 2011;286(13):11218-11225.

235. Eathiraj S, Palma R, Volckova E, Hirschi M, France DS, Ashwell MA, Chan TCK. Discovery of a Novel Mode of Protein Kinase Inhibition Characterized by the Mechanism of Inhibition of Human Mesenchymal-epithelial Transition Factor (c-Met) Protein Autophosphorylation by ARQ 197. J Biol Chem 2011;286(23):20666-20676.

236. Yan SB, Peek VL, Ajamie R, Buchanan SG, Graff JR, Heidler SA, Hui YH, Huss KL, Konicek BW, Manro JR, Shih C, Stewart JA, Stewart TR, Stout SL, Uhlik MT, Um SL, Wang Y, Wu W, Yan L, Yang WJ, Zhong B, Walgren RA. LY2801653 is an orally bioavailable multi-kinase inhibitor with potent activity against MET, MST1R, and other oncoproteins, and displays anti-tumor activities in mouse xenograft models. Invest New Drugs 2013;31(4):833-844.

237. Cui JJ, Tran-Dubé M, Shen H, Nambu M, Kung P-P, Pairish M, Jia L, Meng J, Funk L, Botrous I, McTigue M, Grodsky N, Ryan K, Padrique E, Alton G, Timofeevski S, Yamazaki S, Li Q, Zou H, Christensen J, Mroczkowski B, Bender S, Kania RS, Edwards MP. Structure Based Drug Design of Crizotinib (PF02341066), a Potent and Selective Dual Inhibitor of Mesenchymal-Epithelial Transition Factor (c-MET) Kinase and Anaplastic Lymphoma Kinase (ALK). J Med Chem 2011;54(18):6342-6363.

238. Somoza JR, Koditek D, Villasenor AG, Novikov N, Wong MH, Liclican A, Xing W, Lagpacan L, Wang R, Schultz BE, Papalia GA, Samuel D, Lad L, McGrath ME. Structural, biochemical, and biophysical characterization of idelalisib binding to phosphoinositide 3-kinase delta. J Biol Chem 2015;290(13):8439-8446.

239. Berndt A, Miller S, Williams O, Le DD, Houseman BT, Pacold JI, Gorrec F, Hon W-C, Liu Y, Rommel C, Gaillard P, Ruckle T, Schwarz MK, Shokat KM, Shaw JP, Williams RL. The p110ס crystal structure uncovers mechanisms for selectivity and potency of novel PI3K inhibitors. Nat Chem Biol 2010;6(2):117-124.

240. Furet P, Guagnano V, Fairhurst RA, Imbach-Weese P, Bruce I, Knapp M, Fritsch C, Blasco F, Blanz J, Aichholz R, Hamon J, Fabbro D, Caravatti G. Discovery of NVP-BYL719 a potent and selective phosphatidylinositol-3 kinase alpha inhibitor selected for clinical evaluation. Bioorg Med Chem Lett 2013;23(13):3741-3748.

241. Maira S-M, Stauffer F, Brueggen J, Furet P, Schnell C, Fritsch C, Brachmann S, Chene P, De Pover A, Schoemaker K, Fabbro D, Gabriel D, Simonen M, Murphy L, Finan P, Sellers W, Garcia-Echeverria C. Identification and characterization of NVP-BEZ235, a new orally available dual phosphatidylinositol 3kinase/mammalian target of rapamycin inhibitor with potent in vivo antitumor activity. Mol Cancer Ther $2008 ; 7(7): 1851-1863$. 
242. Liu Q, Wang J, Kang SA, Thoreen CC, Hur W, Ahmed T, Sabatini DM, Gray NS. Discovery of 9-(6aminopyridin-3-yl)-1-(3-(trifluoromethyl)phenyl)benzo[h][1,6]naphthyridin-2( $1 \mathrm{H}$ )-one (Torin2) as a potent, selective, and orally available mammalian target of rapamycin (mTOR) inhibitor for treatment of cancer. J Med Chem 2011;54(5):1473-1480.

243. Yang H, Rudge DG, Koos JD, Vaidialingam B, Yang HJ, Pavletich NP. mTOR kinase structure, mechanism and regulation. Nature 2013;497(7448):217-223.

244. Knight ZA, Gonzalez B, Feldman ME, Zunder ER, Goldenberg DD, Williams O, Loewith R, Stokoe D, Balla A, Toth B, Balla T, Weiss WA, Williams RL, Shokat KM. A pharmacological map of the PI3-K family defines a role for p110alpha in insulin signaling. Cell 2006;125(4):733-747.

245. Fischmann TO, Smith CK, Mayhood TW, Myers JE, Reichert P, Mannarino A, Carr D, Zhu H, Wong J, Yang R-S, Le HV, Madison VS. Crystal Structures of MEK1 Binary and Ternary Complexes with Nucleotides and Inhibitors. Biochemistry 2009;48(12):2661-2674.

246. Iverson C, Larson G, Lai C, Yeh L-T, Dadson C, Weingarten P, Appleby T, Vo T, Maderna A, Vernier JM, Hamatake R, Miner JN, Quart B. RDEA119/BAY 869766: A Potent, Selective, Allosteric Inhibitor of MEK1/2 for the Treatment of Cancer. Cancer Res 2009;69(17):6839-6847.

247. Amidon GL, Lennernas H, Shah VP, Crison JR. A theoretical basis for a biopharmaceutic drug classification: the correlation of in vitro drug product dissolution and in vivo bioavailability. Pharm Res 1995; 12(3):413-420.

248. Kasim NA, Whitehouse $M$, Ramachandran $C$, Bermejo $M$, Lennernas $H$, Hussain AS, Junginger $H E$, Stavchansky SA, Midha KK, Shah VP, Amidon GL. Molecular properties of WHO essential drugs and provisional biopharmaceutical classification. Mol Pharm 2004;1(1):85-96.

249. Hopkins AL, Keseru GM, Leeson PD, Rees DC, Reynolds CH. The role of ligand efficiency metrics in drug discovery. Nat Rev Drug Discov 2014;13(2):105-121. 
Figure 1. Historical overview of kinase inhibitor approvals in oncology.

Figure 2. Active and inactive kinase architectures. An active, i.e. catalytically competent, form of PKA (PDB entry $1 \mathrm{ATP}$ ), bound to ATP, $\mathrm{Mn}^{2+}$ (purple spheres; under physiological conditions the corresponding metal cations are $\mathrm{Mg}^{2+}$ ), and a peptide substrate is shown, with key $\mathrm{H}$-bonds indicated as broken black lines. The side chains of the Phe (R2) and Asp residues of the DFG motif in the A-loop are shown as sticks. The residues making up the regulatory spine are labelled R0 to R4 and the linear arrangement of this spine in active kinase conformations is indicated by a broken line (wheat) connecting these residues. This - and subsequent figures showing 3-dimensional protein structures - were constructed using PyMOL (The PyMOL Molecular Graphics System, Version 1.7, Schrödinger, LLC.).

Figure 3. Kinase inhibition mechanisms and selectivity. (a) Receptor-ligand interaction mechanisms, kinetic parameters, and selectivity indices (adapted from ref. ${ }^{22}$; refer main text for explanations). (b) Relationship between $C_{\text {ssf }}$ and on-target fo for type-I kinase inhibitors that all bind a particular on-target kinase with a $K_{\mathrm{d}}$ value of $10 \mathrm{nM}$ but that bind an off-target kinase with $K_{\mathrm{d}}$ values of $50 \mathrm{nM}\left(S I_{1}=5\right), 250 \mathrm{nM}\left(S I_{1}=25\right), 1 \mu \mathrm{M}\left(S I_{1}=\right.$ $100)$, and $10 \mu \mathrm{M}\left(S I_{1}=1,000\right)$. (c) Relationship between time post $C_{\max }$ and blood levels $\left(C_{\mathrm{f}}\right)$ following p.o. administration of a kinase inhibitor $\left(C_{\max }\right.$ of $10 \mu \mathrm{M}$ and $t_{1 / 2}$ of $3 \mathrm{~h}$ ) that interacts with 3 target kinases with variable rate constants $k_{4}$ of $8.5 \times 10^{-3} \mathrm{~s}^{-1}$ (target 1 ), $2.8 \times 10^{-4} \mathrm{~s}^{-1}$ (target 2), and $1.26 \times 10^{-5} \mathrm{~s}^{-1}$ (target 3) and identical $k_{1}$ of $1 \times 10^{8} \mathrm{M}^{-1} \mathrm{~s}^{1}, k_{2}$ of $1 \mathrm{~s}^{-1}$, and $k_{3}$ of $6 \times 10^{-3} \mathrm{~s}^{-1}$, giving residence times $(T)$ of 2 min (target 1 ), $1 \mathrm{~h}$ (target 2 ), and $22 \mathrm{~h}$ (target 3 ).

Figure 4. Small-molecule kinase inhibitors in the clinic. The numbers of approved agents and those currently undergoing clinical evaluation is summarised by kinase.

Figure 5. Molecular basis of BCR-ABL resistance. (a) The chemical structures of $1^{\text {st }}$ (imatinib), $2^{\text {nd }}$ (dasatinib), and $3^{\text {rd }}$ generation (ponatinib) ABL inhibitors. (b) The ABL kinase (green) binding mode of the type-II inhibitor imatinib (grey;) shows an H-bond interaction (dashed line) with the gatekeeper residue T315 (yellow; secondary mutation sites Y253 \& E255 in the Gly-rich loop are indicated in purple). ${ }^{222}$ (c) The binding of ATP does not involve the T351 residue, hence the catalytic competence of the T315I mutant. ${ }^{223}$ (d) Second-generation drugs such as the type-I compound dasatinib are not effective against T315I mutant kinase, as they also make polar interactions with T315. ${ }^{224}$ (e) The recently approved ponatinib, which also inhibits the T315I mutant, evades the altered gatekeeper (Ile) through the placement of an ethynyl linkage between its front- and back-pocket binding portions. ${ }^{225}$ Constructed from PDB entries 1IEP, 2SRC, 2GQG, and 3IK3.

Figure 6. Non-ATP-dependent SRC kinase inhibition. (a) The substrate peptide SRC kinase inhibitor KX 01 was shown by saturation transfer difference NMR spectroscopy, using an ATP site-binding paramagnetic spinlabelled pyrazolopyrimidine probe containing the 2,2,6,6-tetramethyl-piperidine-1-oxyl (TEMPO) radical, to bind to SRC kinases in an ATP non-competitive manner. ${ }^{226}$ (b) An X-ray crystal structure (PDB entry $3 K X Z$ ) of this probe (green) bound to the SRC-family kinase LCK (surface with electrostatic potential colouring) is shown. The extended substrate-binding cavity, where KX-01 (cyan, modelled (Schrödinger Glide, https://www.schrodinger.com/Glide) binding pose) is likely to bind, is evident proximal to the ATP-binding site. 
Figure 7. Inhibition of EGFR Tyr kinases. (a) Chemical structures of inhibitors. (b) Erlotinib (cyan) is a HER1selective inhibitor; it binds to HER1 (grey cartoon; G-loop in blue) with the protein in an active-like conformation, with an extended A-loop (yellow) and inward position of the aC-helix (magenta). ${ }^{227}$ The phenylacetylene group of erlotinib interacts with the side chain of M766 (magenta stick model) at the base of the aC-helix, as well as the gatekeeper residue T790 (green). (c) Similarly, the HER2-selective compound SYR 127063 binds to HER2 in a way that involves an active-like conformation of that protein. Here the ligand trifluoromethylphenyl and chloro groups interact with M766 (EGFR numbering) and T790, respectively. ${ }^{228}$ It is evident that in active HER2 the inward position of the aC-helix is less pronounced than in active HER1. In active HER2 the cavity proximal to the gatekeeper is thus larger than in active HER1 and can accommodate the larger bis(aryl)ether group of SYR 127063 , whereas the phenylacetylene group of erlotinib would be unable to make contacts with the aC-helix in HER2. Conversely the bis(aryl)ether group of SYR 127063 is too large to fit the gatekeeper-aC-helix cavity of active EGFR. (d) Dual HER1 and HER2 inhibitors such as lapatinib are generally observed to bind preferentially to inactive forms of the protein (HER1 shown), in which a short a-helix in the A-loop supports an outward position of the aC-helix. ${ }^{229}$ (e) TAK 285 is the only EGFR inhibitor for which crystal complexes with both HER1 (ligand and coloured protein elements in light colouring) and HER2 (dark colouring) have been solved. ${ }^{228,230}$ Although similar in structure to SYR 127063, TAK 285 inhibits HER1 and HER2 with similar potency. It can be seen that it binds to inactive forms of both kinases, which are highly similar. (f) Second-generation type-VI inhibitors are active against T790M mutant kinase forms and e.g. afatinib binds to wild-type (ligand and coloured protein elements in light colouring) and the T790M form of HER1 (dark colouring) in a very similar manner, with slight repositioning of the substituted aniline to optimise interactions with T790 or T790M. ${ }^{68}$ (g) Osimertinib, on the other hand, is a type-VI inhibitor that selectively binds to the T790M mutant EGFR kinase. It was observed that the main determinant for this selectivity in the design of osimertinib was the indole N-methyl group, which can be observed to interact closely with the thiomethyl group of M790, an interaction that is not possible with T790 (modelled pose from docking using the Schrödinger Glide programme with the covalent docking protocol, using the 3IKA HER1 structure as the receptor). ${ }^{231}$ Constructed from PDB entries 1M17, 3PP0, 1XKK, 3POZ, 3RCD, 4G5J, and 4G5P.

Figure 8. Experimental binding modes of FGFR inhibitors. (a) Chemical structures of inhibitors. (b) The three clinically most advanced selective FGFR inhibitors AZD 4547 (cyan), infigratinib (magenta), and erdafitinib (green) all contain 3,5-dimethoxyphenyl groups that make identical interactions with the kinase. (c) Lucatinib (green) and dovitinib (cyan) are MTKIs that also inhibit FGFR kinases with high potency. Lucatinib makes contacts with the V561 gatekeeper residue (spheres), whereas dovitinib does not. (d) For this reason dovitinib inhibits both wild-type (cyan) and V561M-mutated (green) forms of the kinase, whereas lucatinib is inactive against V561M FGFR. ${ }^{89}$ (e) BLU 9931 (green) is an isoform-selective type-VI FGFR4 inhibitor ${ }^{232}$ by virtue of targeting C552, which is unique in FGFR4 (Tyr in FGFR1-3), whereas FIIN-2 ${ }^{233}$ is a selective dual inhibitor of FGFR and HER kinases, both of which contain Cys residues at the same position in the G-loop (C447 in FGFR, C797 in HER). Figure constructed from PDB entries 4RWJ, 5EW8, 3TT0, 4RWL, 4RWI, 5AM6, 5AM7, 4XCU, and 4QQC.

Figure 9. Inhibitors of active and inactive forms of MET. (a) Chemical structures of inhibitors. (b) The Gloop in the N-terminal lobe of the apo-form of MET $^{234}$ is shown in cyan, the aC-helix, which contains key catalytic residues, is shown in magenta, and the A-loop, which is important for substrate recognition, in yellow, with the Phe residue of the DFG motif shown as a stick model. An example of an inhibitor that is selective for A-loopphosphorylated active form of MET is the derivative of MK 2461. It is a type-I inhibitor and the shape of the ATPbinding site in its complexed structure (c) is similar to that of the apo-form, except that the G-loop folds over the ligand. ${ }^{234}$ The late-stage development Met inhibitor tivantinib is an unusual type-II inhibitor, which does not bind deep into the cavity formed by the DFG-out conformation, but it induces a major shape change in the kinase, 
involving both the A-loop and the aC-helix (d). ${ }^{235}$ Like tivantinib, merestinib is selective for unphosphorylated inactive MET; it is a conventional type-II inhibitor (e). ${ }^{236}$ Constructed from PDB entries 3Q6U, 3Q6W, 3RHK, and 4EEV.

Figure 10. ALK inhibitors. (a) Chemical structures of crizotinib and lorlatinib, a macrocyclic derivative with high lipophilic efficiency and CNS bioavailability. (b) Crizotinib (green) and lorlatinib display highly similar ALK binding modes (crystallographic water molecules shown as spheres, $\mathrm{H}$-bonds as broken lines)..$^{99,237}$ Constructed from PDB entries 2 XP2 and 4CLI.

Figure 11. The basis of selectivity of PI3K and mTOR inhibitors. (a) Chemical structures of inhibitors. (b) The PI3Kס-selective idelalisib ${ }^{238}$ (green) binds to PI3Kס through H-bonding interactions (broken lines) with the backbone amide bonds of the hinge region (cyan). Its selectivity derives predominantly from induced-fit binding of the quinazolinone substructure between the side chains of M752 and W760 (spheres). (c) An apo structure of PI3K $\delta^{239}$ shows that the residues that differ between the four PI3K isoforms (magenta) are quite remote from the ligand in the PI3Kס-idelalisib complex (and other ligands in other complexes) and that the slot between the M752 and W760 residues is closed. Although the residues corresponding to M752 and W760 are conserved in all 4 PI3K isoforms, induced-fit binding as observed with idalalisib appears to be a selectivity feature of PI3Kס-selective compounds. $^{239}$ (d) The PI3Ka-selective alpelisib ${ }^{240}$ interacts closely (broken lines) with the side chain of Q859, one of the few residues around the ATP-binding pocket that are not conserved across the PI3Ks. (e) The panPI3K-selective buparlisip ${ }^{241}$ binds to PI3Ky in a similar mode as alpelisib binds to PI3K $\delta$ but any selectivityimparting features are absent. (f) The mTOR-selective compound torin $2^{242}$ binds to mTOR without any of the usual hinge-region $\mathrm{H}$-bonds. Its selectivity is probably due in large part to interaction of the naphthyridinone substructure with the indole of W2239 in the hinge, which is not conserved in PI3Ks. ${ }^{243}$ (g) The dual mTOR- and pan-PI3K inhibitor PI $103^{244}$ interacts with mTOR through polar interactions involving the morpoline $O$ and the phenol $\mathrm{OH}$ groups. ${ }^{243}$ Similar interactions are also possible with any of the PI3K isoforms. Furthermore, PI 103 adopts an almost completely planar conformation, which can be accommodated in the mTOR and the PI3K ATPbinding sites, regardless of the width of the binding clefts. Constructed from PDB entries 4XE0, 2WXR, 4JPS, 3SD5, 4JSX, and 4JT6.

Figure 12. Type-I and type-III AKT inhibitors. (a) AZD 5363 and ipatasertib are type-I inhibitors, whereas inhibitor VIII ${ }^{157}$ and MK 2206 are type-III inhibitors. (b) Compound VIII (salmon) binds to AKT (N-lobe dark cyan, $\mathrm{C}$-lobe magenta, $\mathrm{PH}$ domain yellow) in an inactive conformation compared to the active state as represented by the superposed complex between AMP-PNP (green) and AKT1 (N-lobe light cyan, C-lobe grey). Compound VIII binds at a site adjacent to the ATP-binding site composed of portions of the $\mathrm{N}$ - and $\mathrm{C}$-lobes, as well as the $\mathrm{PH}$ domain. Binding induces or stabilises an inactive kinase conformation with disordered aC-helix, altered N- and Clobes, and an A-loop conformation that projects the F293 side chain of the DFG motif into the ATP-binding site (Phe side chain as spheres). (c) AZD 5363 (magenta) and ipatasertib (cyan) bind to active AKT conformations and in complex with AKT occupy the same space as ATP (AMP-PNP; green). MK 2206 (yellow; predominant pose obtained from Schrödinger Glide docking shown) probably binds in a very similar manner to AKT as compound VIII (salmon). Constructed from PDB entries 3096, 4EKK, 4EKL, and 4GV1.

Figure 13. Structural basis of MEK inhibition. MEK1 and MEK2 are comparatively unique insofar as these kinases contain an allosteric binding site adjacent to, but not overlapping, with the ATP-binding pocket. This exosite is evident in ATP/ $\mathrm{Mg}^{2+}$-bound structures (a), as well as in complexes with type-I inhibitors (e.g. K252a, 
b). ${ }^{245} \mathrm{~A}$ number of ATP non-competitive MEK inhibitors have been discovered that occupy this exosite, thus distorting the catalytic site and inactivating the enzyme (e.g. rafametinib (grey), superposed with ATP (cyan), c) ${ }^{246}$ The 2-fluoro-4-iodo-aniline system of rafametinib has high shape complementarity with the lipophilic backpocket of the exosite (d) and is in fact present in most known type-III MEK inhibitors, including 7 of 8 current clinical MEK inhibitors (e). Protein structures are shown as green surfaces or meshes; ATP and related nucleosides are shown as cyan sticks ( $\mathrm{Mg}^{2+}$ as green spheres), allosteric inhibitors as grey sticks, and $\mathrm{K} 252 \mathrm{a}$ as yellow sticks. Constructed from PDB entries 1S9], 2P55, 3DV3, 3DY7, 3E8N, 3EQB, 3EQC, 3EQD, 3EQF, 3EQG, 3EQH, 3EQI, 3MBL, 3ORN, 30S3, 3PP1, 3SLS, 3V01, 3V04, 4AN2, 4AN3, 4AN9, 4ANB, and 1S9I.

Figure 14. Predicted biopharmaceutical properties of kinase inhibitors compared to all approved drugs. According to the Biopharmaceutics Classification System (BCS), ${ }^{247}$ oral drugs belong to class I if they are highly permeable and highly soluble, class II if highly permeable but of low solubility, class III if poorly permeable but highly soluble, and class IV if both poorly permeable and soluble. For the purposes of bioequivalence and other preclinical and clinical drug development studies class boundaries are based on solubility of the highest dose strength and permeability as assessed by pharmacokinetic comparison of oral and intravenous dosing. Here an attempt is made to predict such classifications based on calculated solubility (logs derived from intrinsic aqueous molar solubility) and permeability ( $\log \mathrm{P}$; predicted octanol-water partition coefficient). LogS and $\log \mathrm{P}$ values of $-4.52(30 \mu \mathrm{M})$ and 1.35 , respectively, were used to set the class boundaries. ${ }^{248}$ It can be seen that using this approximation it is predicted that the majority of kinase inhibitors fall into class II, some into class I, a few into class III, and very few into class IV.

Figure 15. Chemical similarity of oncology kinase inhibitors and all approved drugs. Dissimilarity was calculated using Tanimoto distances based on ChemAxon chemical fingerprints (CF; http://www.chemaxon.com/jchem/doc/user/). Only inhibitor groups of $\geq 5$ compounds are included in the analysis.

Figure 16. Maximum common substructure (MCS) clustering of clinical kinase inhibitors. The chemical structures of the 172 experimental kinase inhibitor drugs were submitted to hierarchical MCS clustering using the LibMCS programme (www.chemaxon.com). The dendrogram shows that this analysis results in 44 top-level clusters, of which 1 has 5 levels, 10 have 3 levels, 25 have 2 levels, and 7 have 1 level. For top-level clusters containing 3 or more common substructures, these are shown.

Figure 17. Fragment-based discovery of the AKT inhibitor AZD 5363. (a) Fragment screening against AKT2 identified 7-azapurine as a promising hinge-binding starting point. (b) Fragment growing led to the benzylamine-substituted purine intermediate 1 and (c) replacement of the phenyl group with piperidine afforded the hydrophilic intermediate 2. (d) Modulation of polarity and further fragment growing yielded the highly active intermediate 3, from which (e) AZD 5363 itself was derived. (f) Fragment growing was guided by the known binding mode of the pharmacophorically related isoquinoline-5-sulfonamide CCT 077373. pIC 50 values refer to AKT2 inhibition. Predicted logP values were calculated using Instant JChem (www.chemaxon.com). Ligand efficiency (LE) was calculated from PIC $_{50}$ values and lipophilic ligand efficiency (LLE) values were calculated from $\mathrm{pIC}_{50}$ and predicted logP. ${ }^{249}$ Binding mode illustrations were constructed from PDB entries 2JDO, 2UVX, 2UVY, 2VNW, 2X39, and 4GV1. Ligands are shown as cyan stick models, $\mathrm{H}$-bonds as broken yellow lines, and ligand $\mathrm{H}-$ bonded water molecules as red spheres. Hinge-region residues involved in ligand $\mathrm{H}$-bonding, charged residues in the ribose-binding region involved in polar ligand interactions, as well as a mobile Phe residue in the G-loop are shown as line models. 
Table 1. Comparative structural and physicochemical properties of kinase inhibitor experimental drugs. ${ }^{a}$

\begin{tabular}{lcc}
\hline Parameter $^{b}$ & $\begin{array}{c}\text { Oncology kinase } \\
\text { inhibitors }\end{array}$ & $\begin{array}{c}\text { All approved } \\
\text { drugs }^{d}\end{array}$ \\
\hline$M_{r}$ & $475 \pm 95(464)$ & $374 \pm 121(351)$ \\
nRot & $6.2 \pm 2.6(6)$ & $5.6 \pm 3.9(5)$ \\
HBA & $6.1 \pm 2.0(6)$ & $4.7 \pm 2.9(4)$ \\
HBD & $2.2 \pm 1.0(2)$ & $1.9 \pm 1.9(1)$ \\
TPSA $\left(\AA^{2}\right)$ & $100 \pm 31(94)$ & $82 \pm 54(73)$ \\
nRing & $4.5 \pm 1.1(4)$ & $2.9 \pm 1.5(3)$ \\
nAr & $3.4 \pm 1.0(3)$ & $1.6 \pm 1.1(2)$ \\
nChir & $0.7 \pm 1.8(0)$ & $2.1 \pm 3.0(1)$ \\
$\operatorname{logP}$ & $3.4 \pm 1.6(3.5)$ & $2.1 \pm 2.8(2.5)$ \\
$\log D_{6.5}$ & $2.6 \pm 1.7(2.7)$ & $0.7 \pm 3.5(1.2)$ \\
$\operatorname{logS}$ & $-5.4 \pm 1.3(-5.4)$ & $-3.7 \pm 2.0(-3.8)$
\end{tabular}

Values are average \pm standard deviation (median). ${ }^{b}$ nRot, number of nonterminal rotatable bonds; HBA, number of H-bond acceptors; HBA, number of H-bond donors; TPSA, topological polar surface area; nRing, number of rings; $\mathrm{nAr}$, number of aromatic rings; $\mathrm{nChir}$, number of chiral centres; log $\mathrm{P}$, predicted octanol-water partition coefficient; $\log D_{6.5}$, predicted octanol-water distribution coefficient at $\mathrm{pH}=6.5$; log $\mathrm{S}$, predicted intrinsic molar aqueous solubility. ${ }^{c}$ Oncology kinase inhibitors, $\mathrm{n}=172 ;{ }^{d}$ all approved drugs, $\mathrm{n}=1,478$ (data from http://www.drugbank.ca/), for small-molecule drugs with $200<M_{\mathrm{r}}<800$, excluding inorganics, neutraceutics, and illicit drugs). TPSA, $\log P, \log D$, and $\log S$ were predicted using the Instant JChem application (https://www.chemaxon.com/products/instant-jchem-suite/instant-jchem/). 


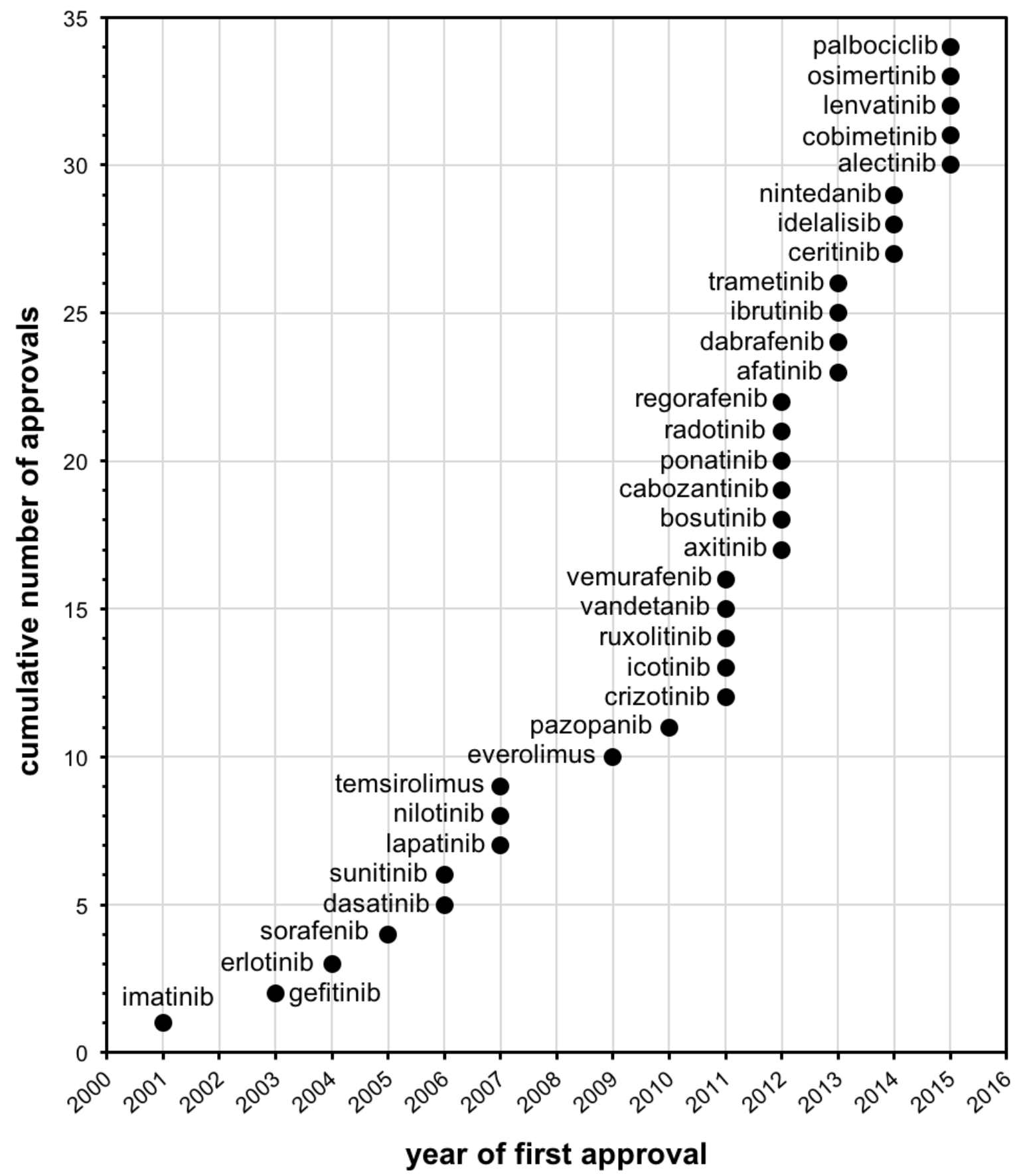

Figure 1 


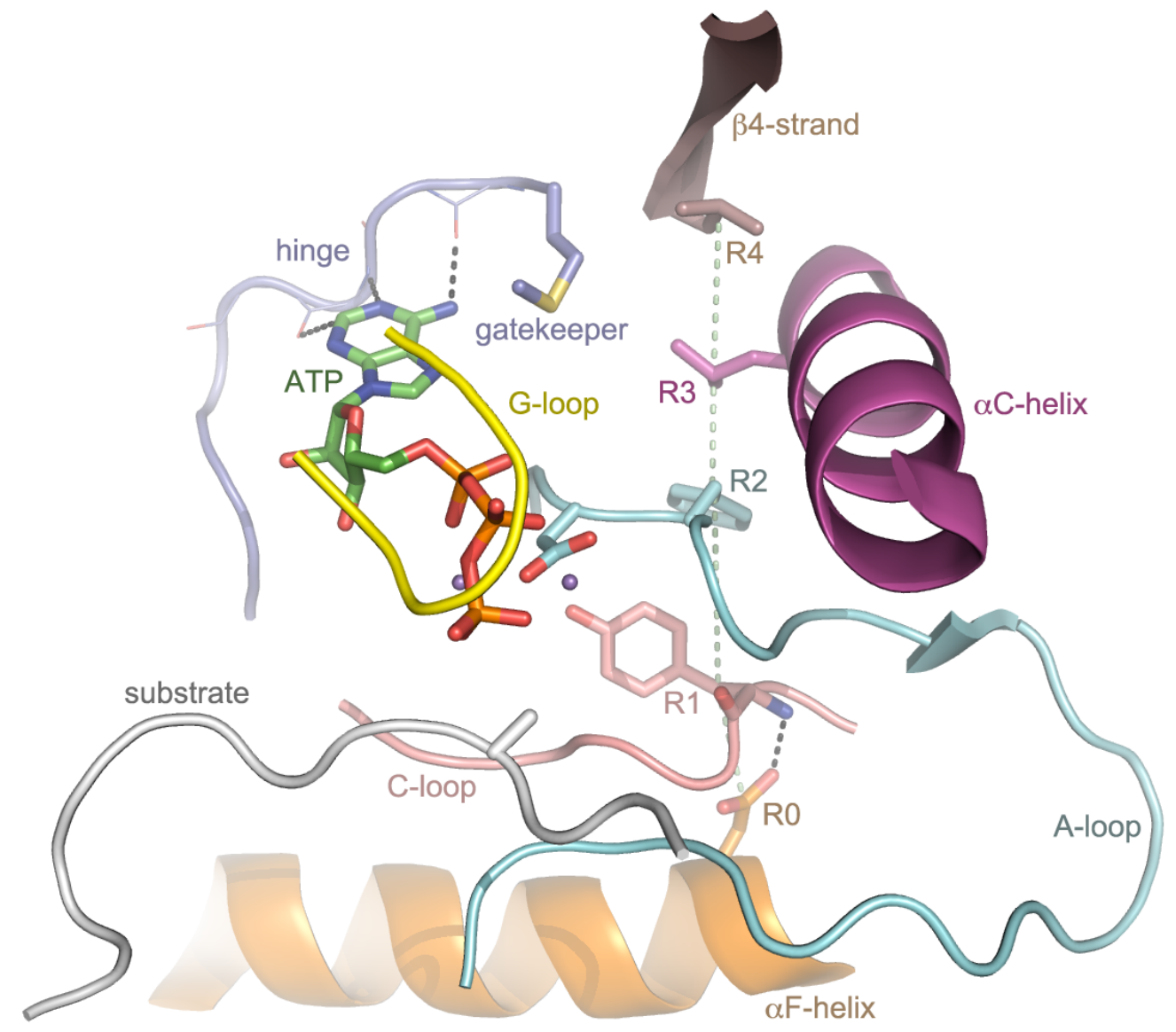

Figure 2 


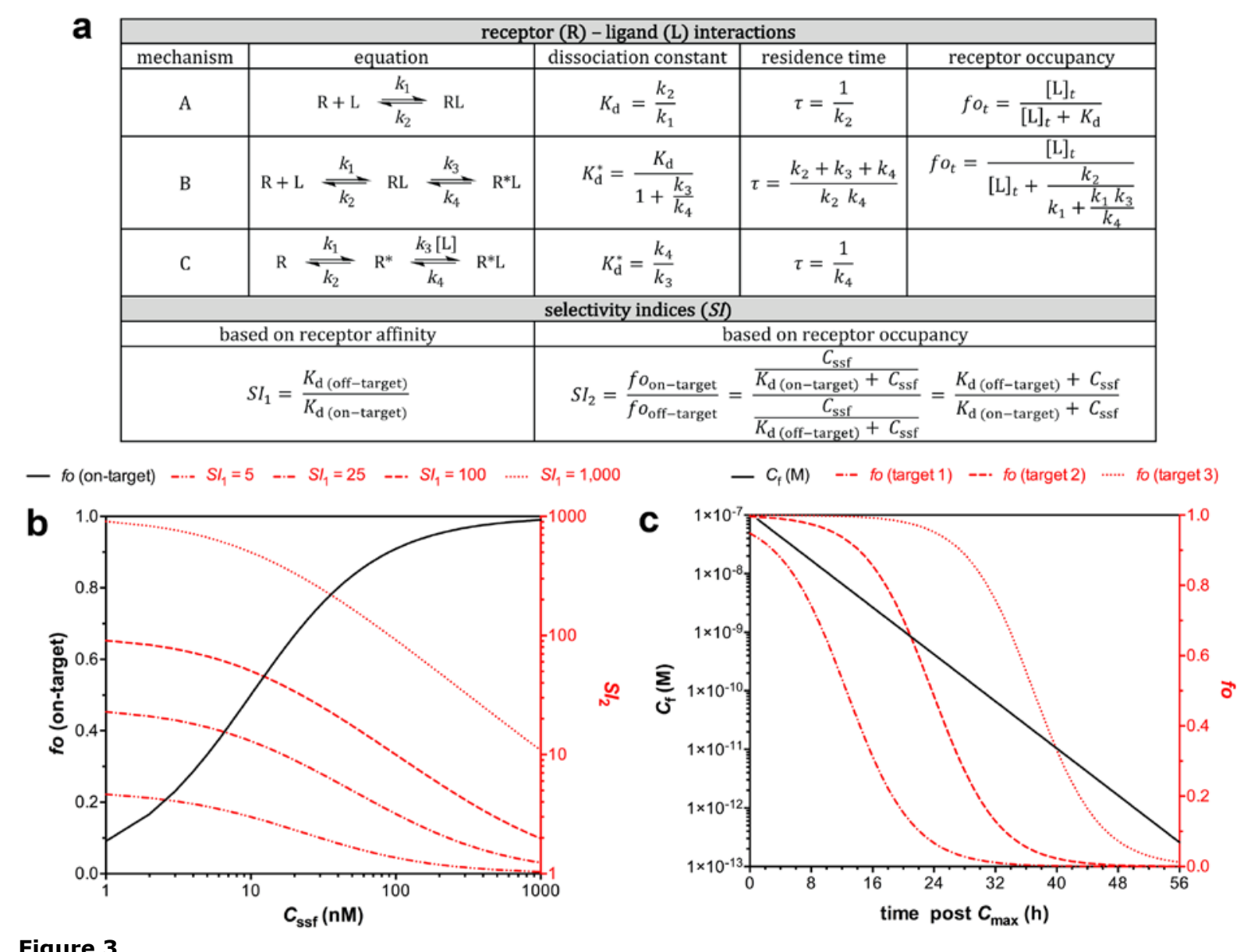

Figure 3 


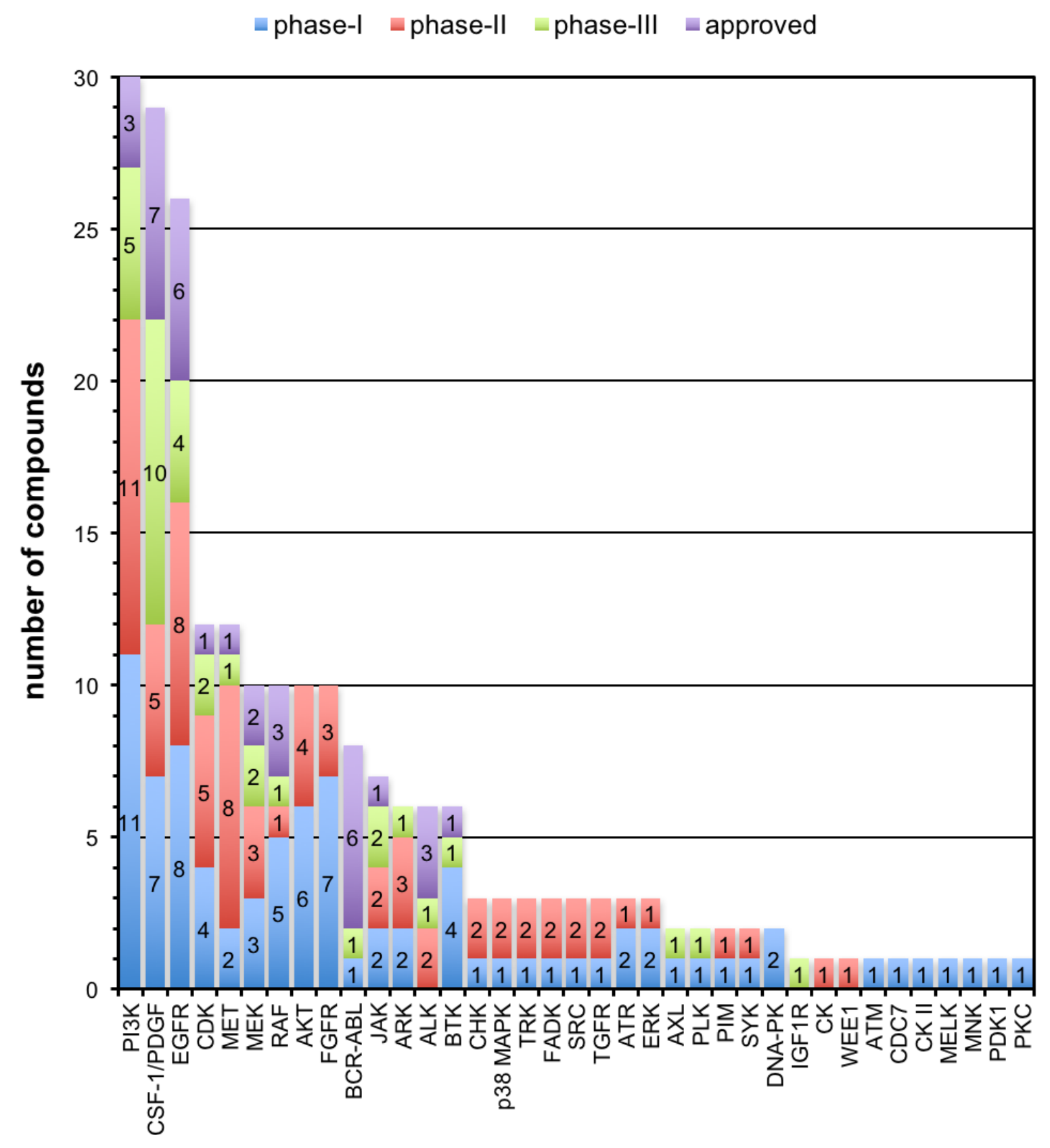

target kinase group

Figure 4 

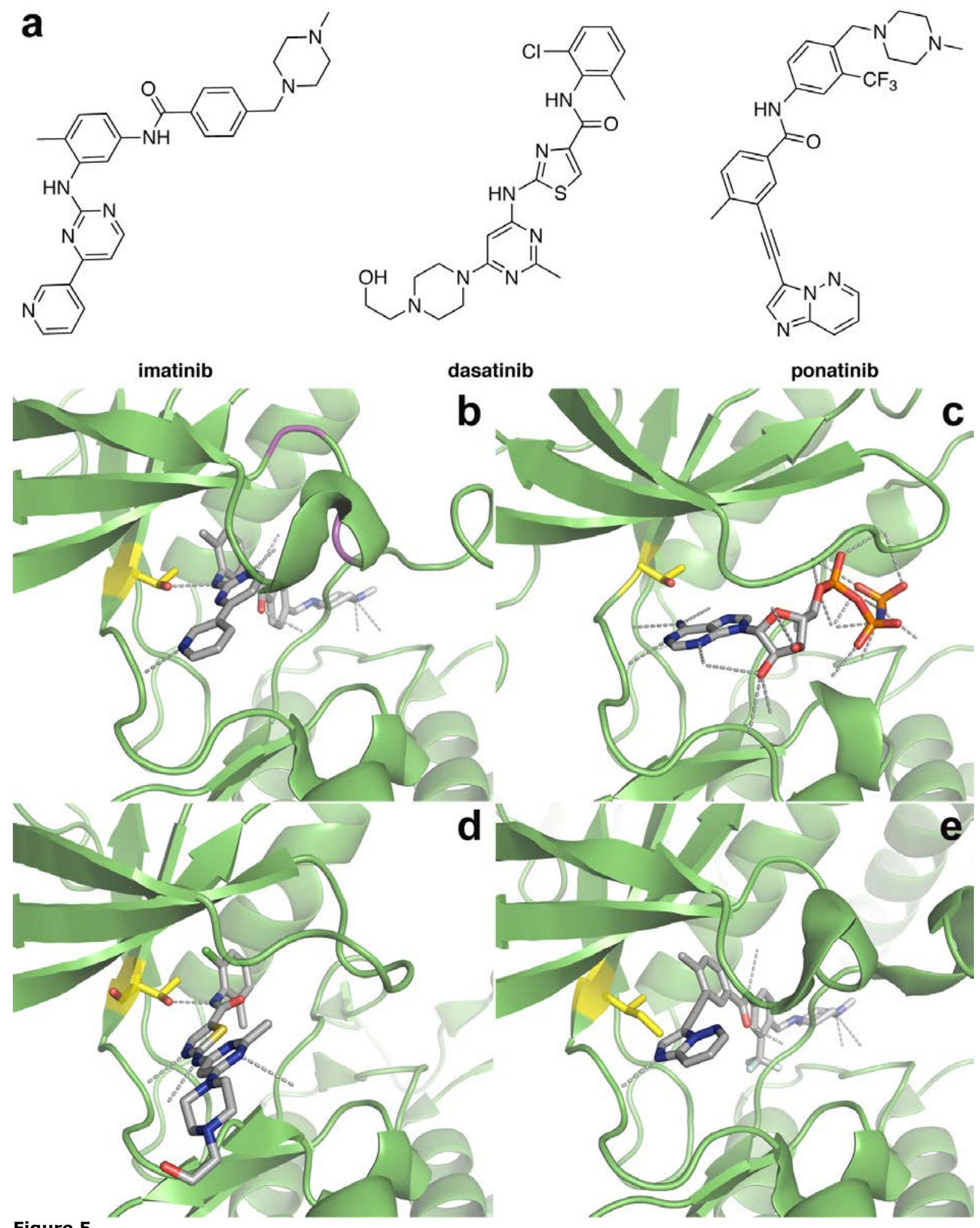


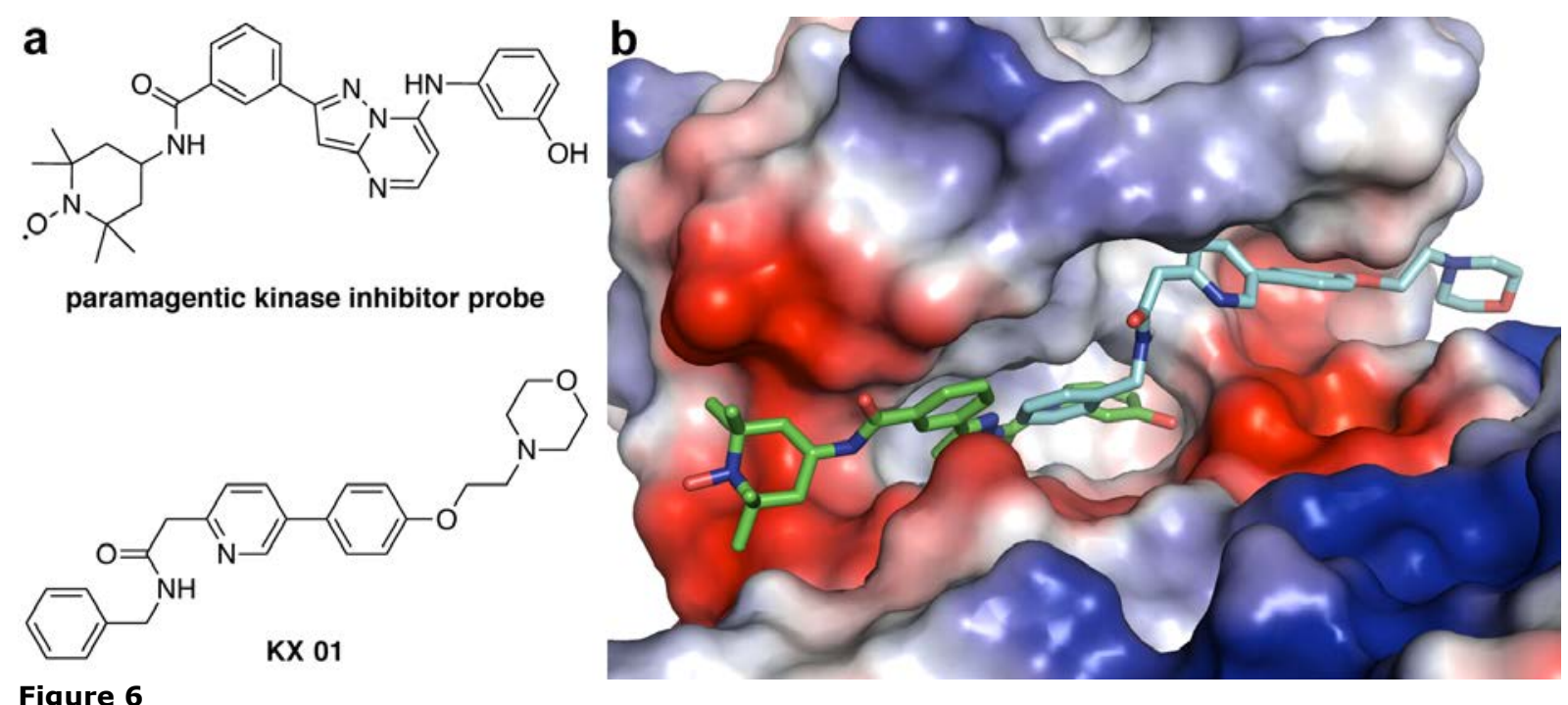

Figure 6 


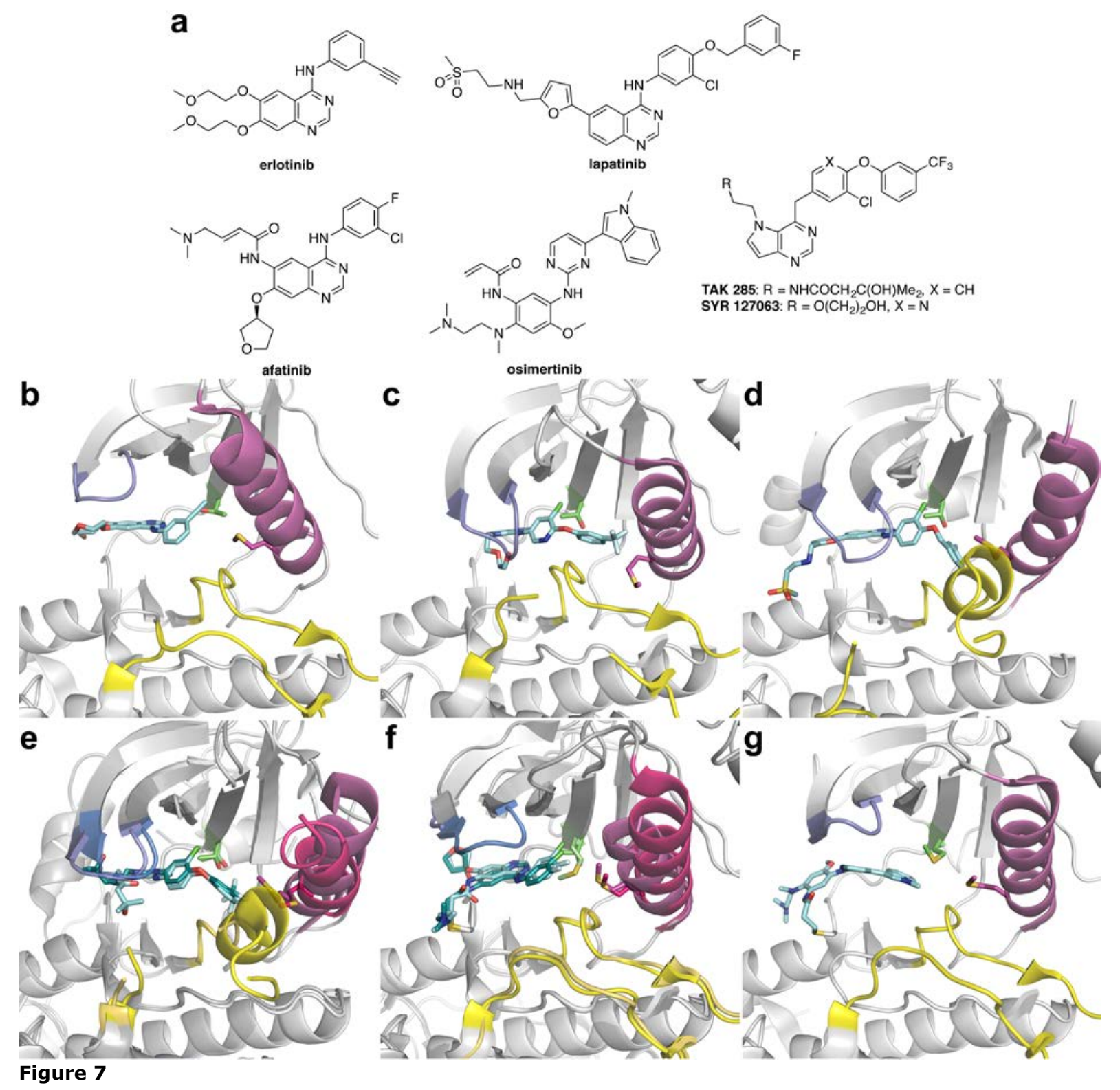




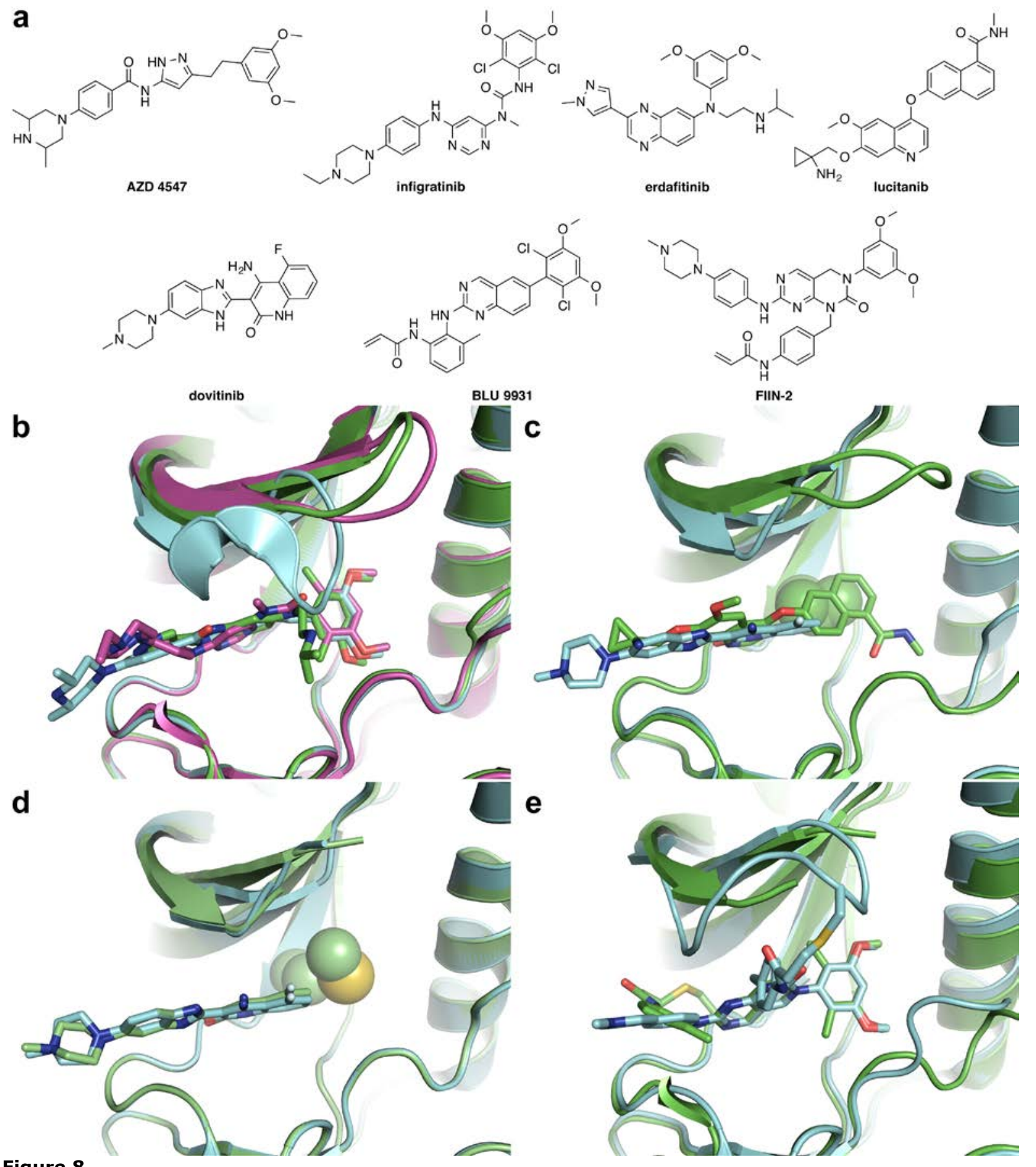

Figure 8 


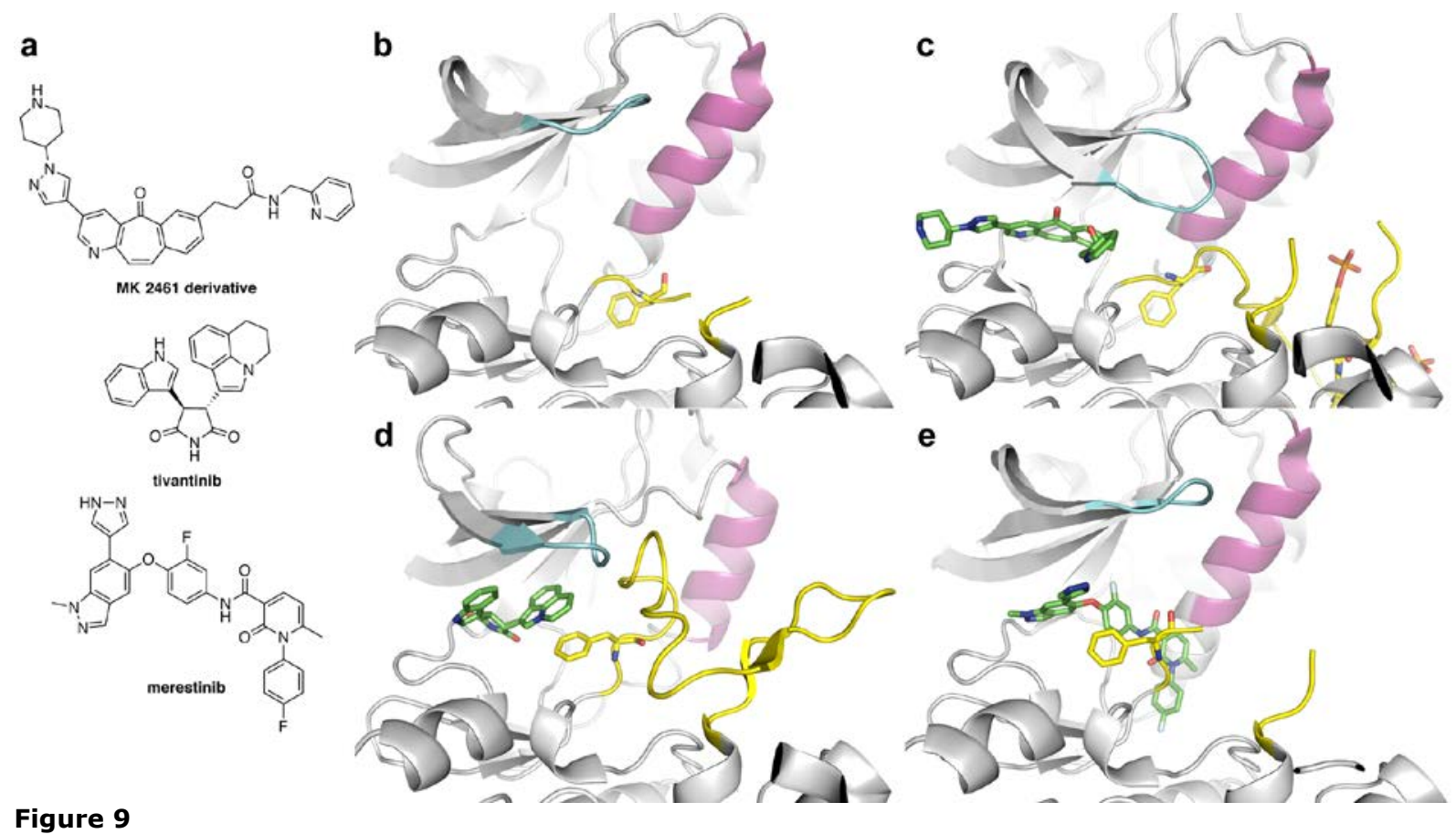




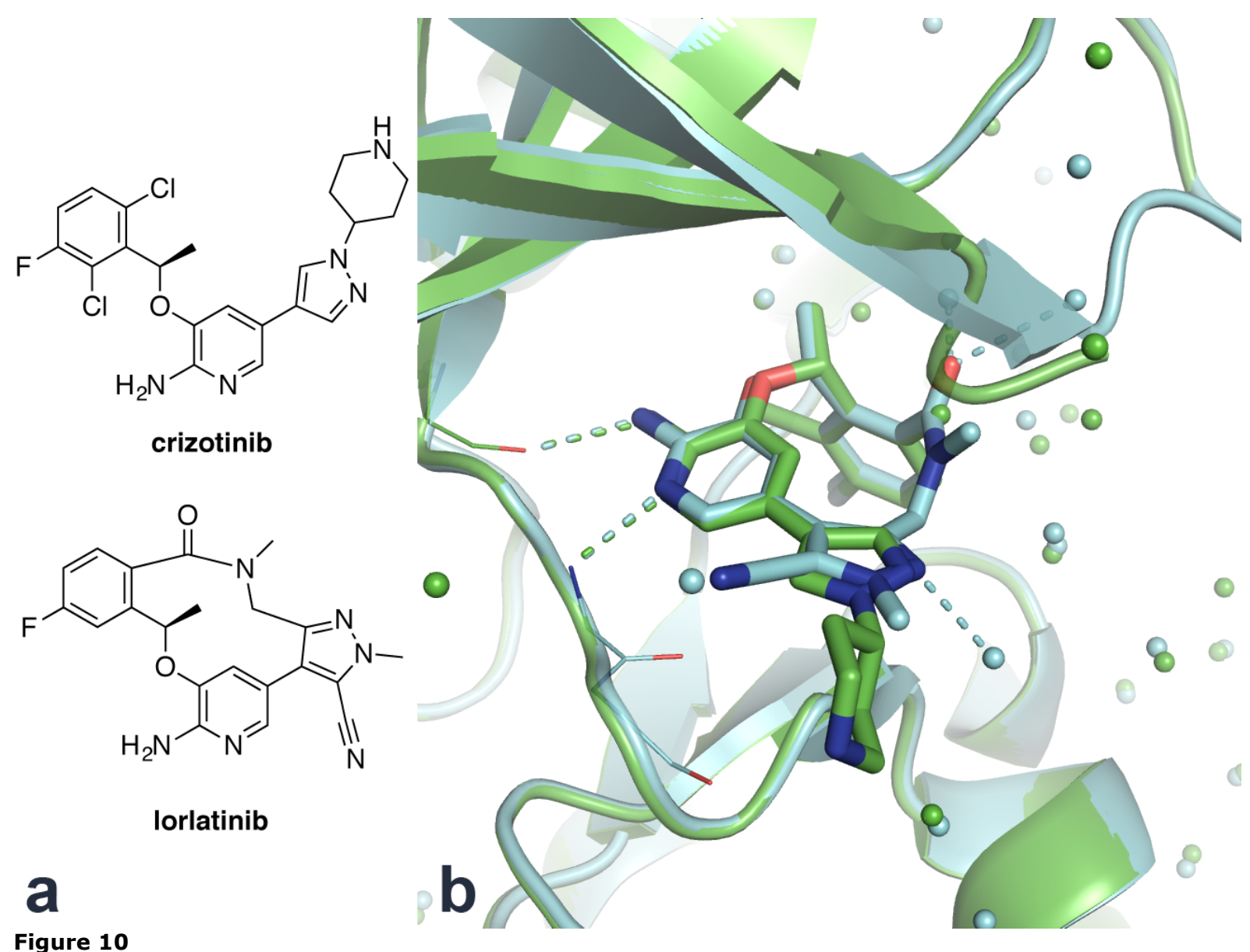

Figure 10 


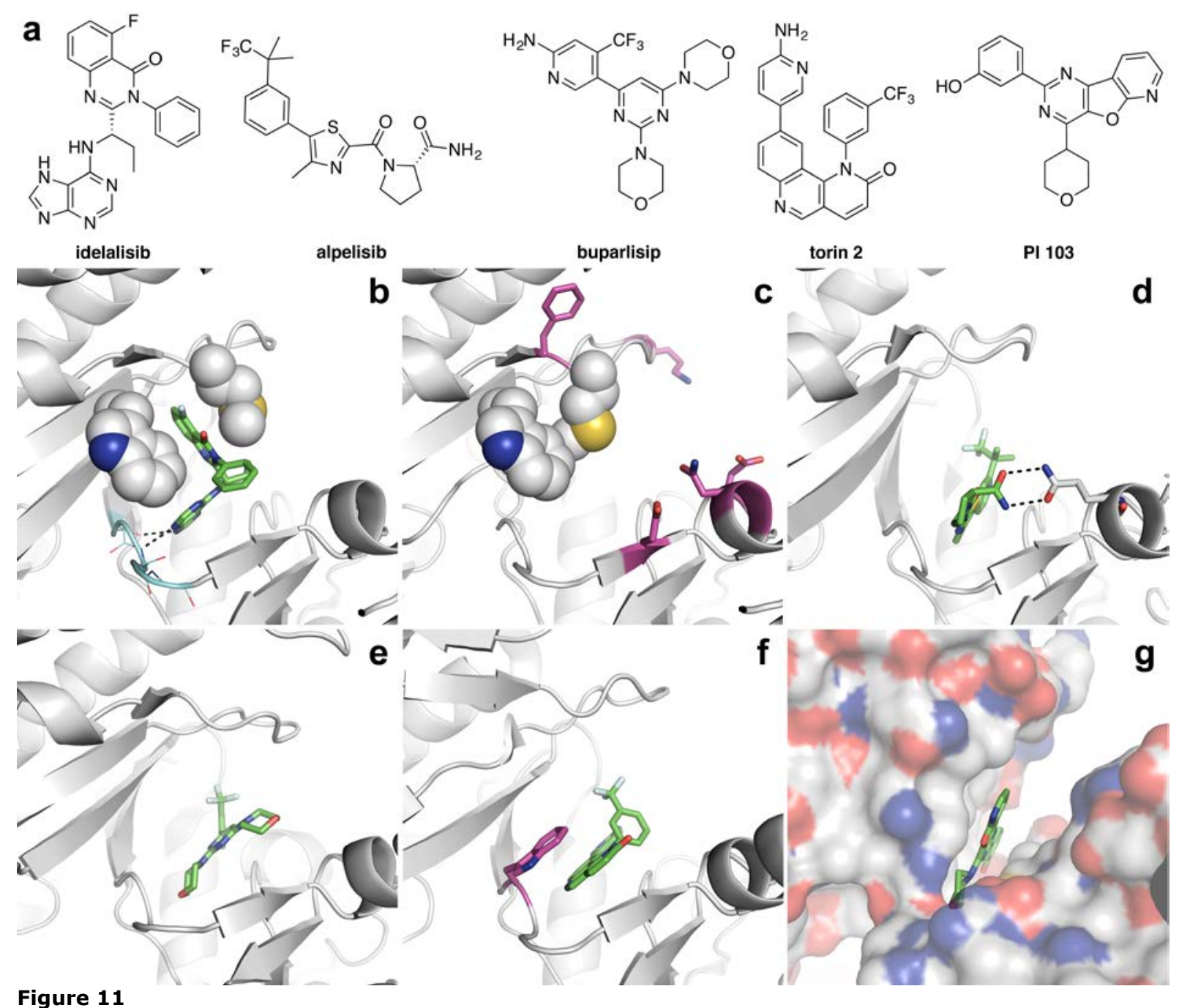

Figure 11 


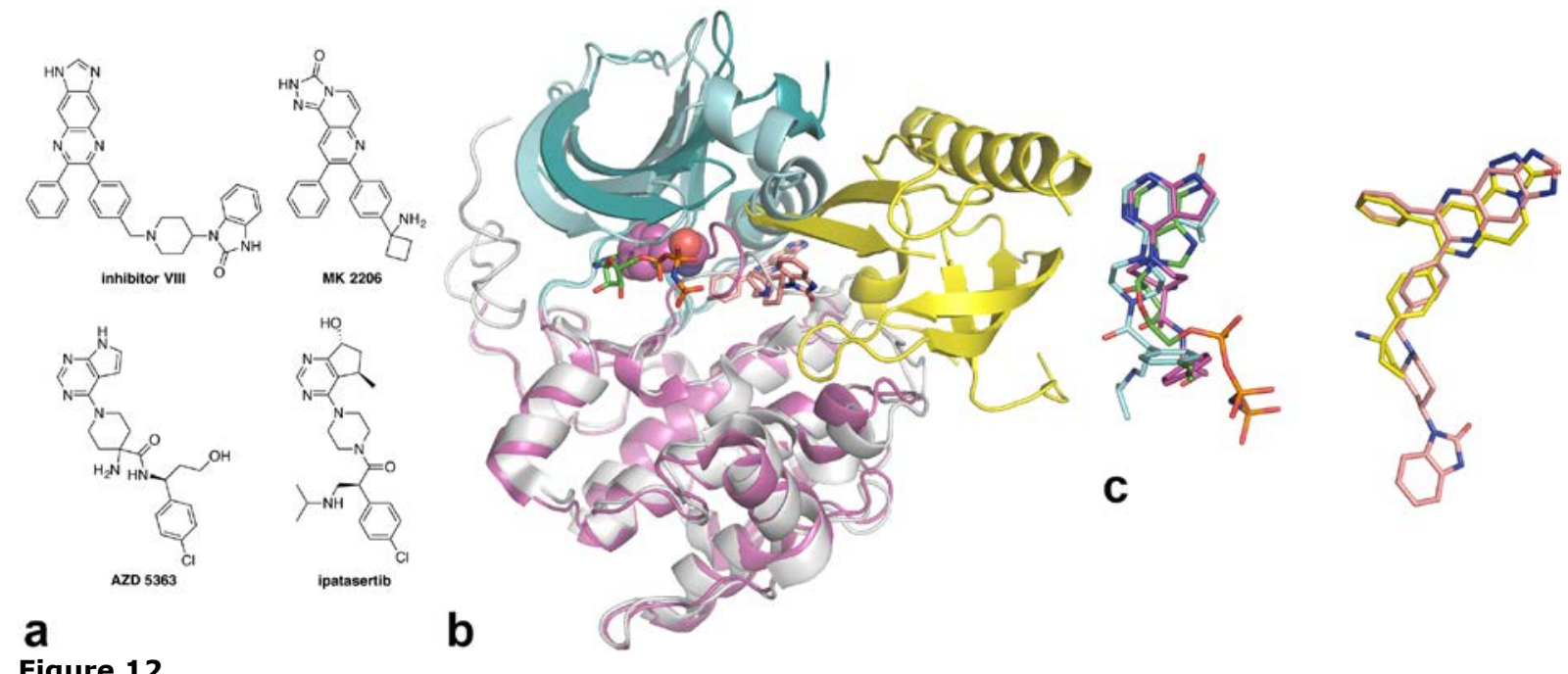

Figure 12 

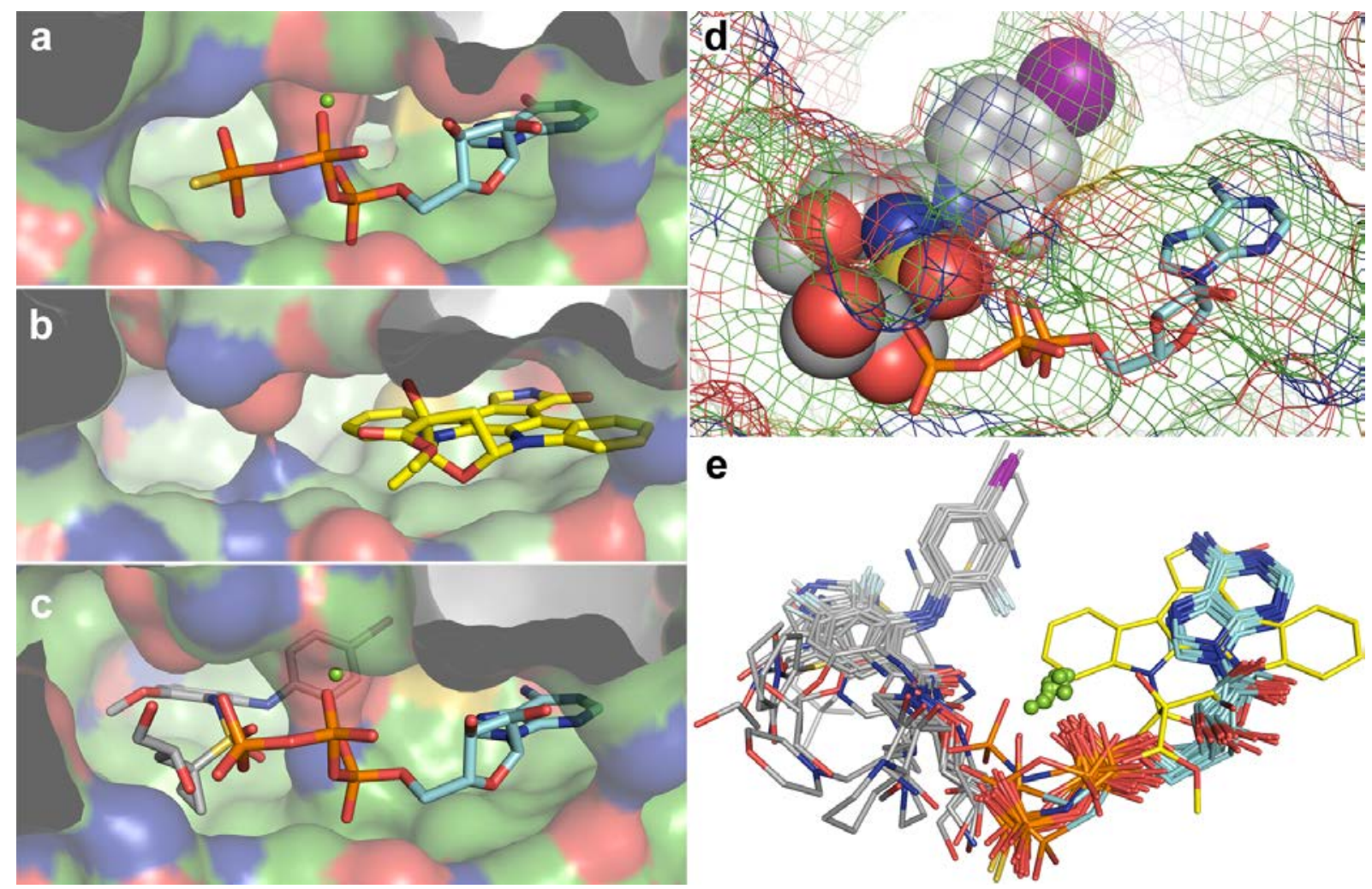

Figure 13 


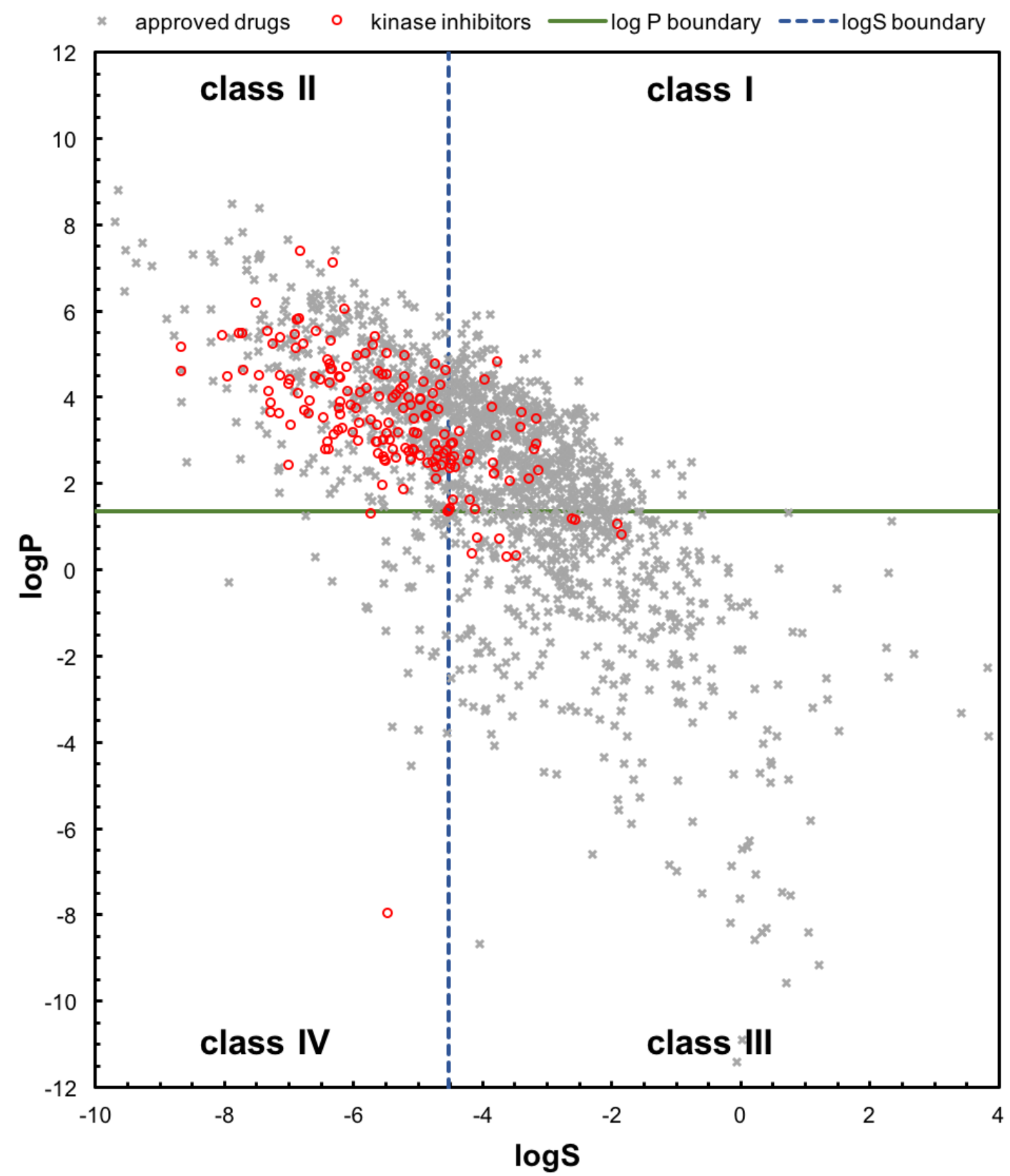

Figure 14 


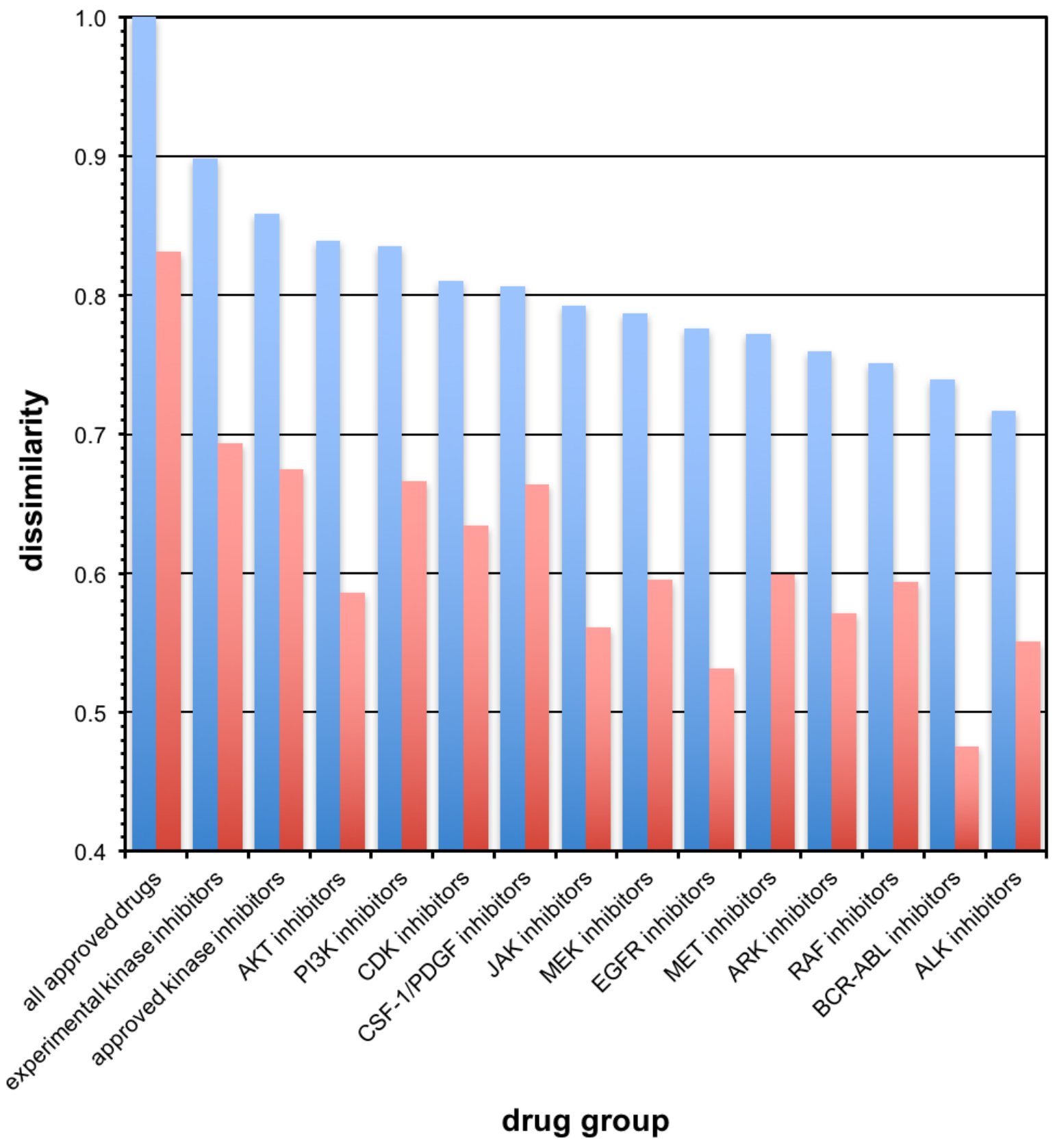

Figure 15 


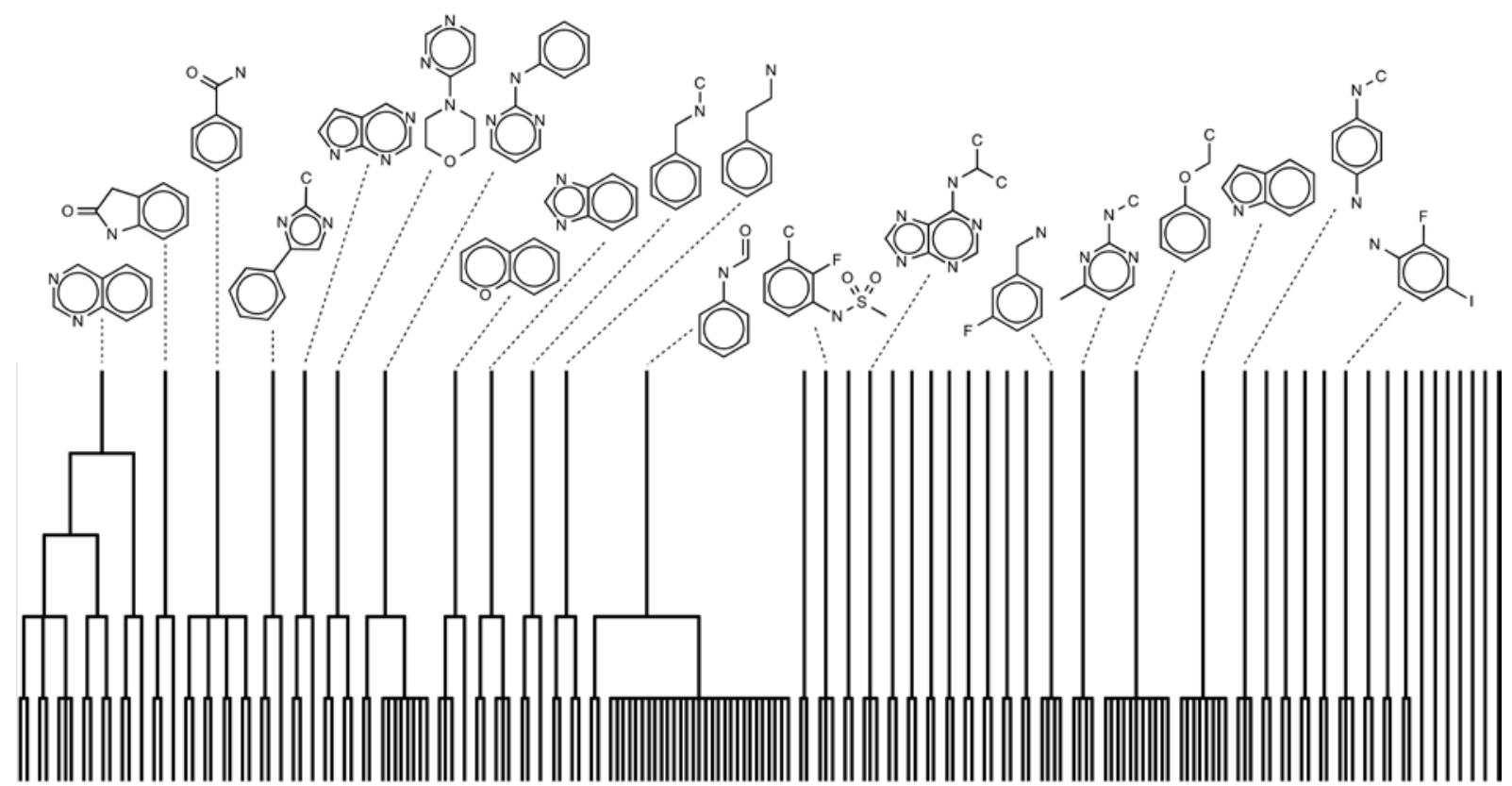

Figure 16 


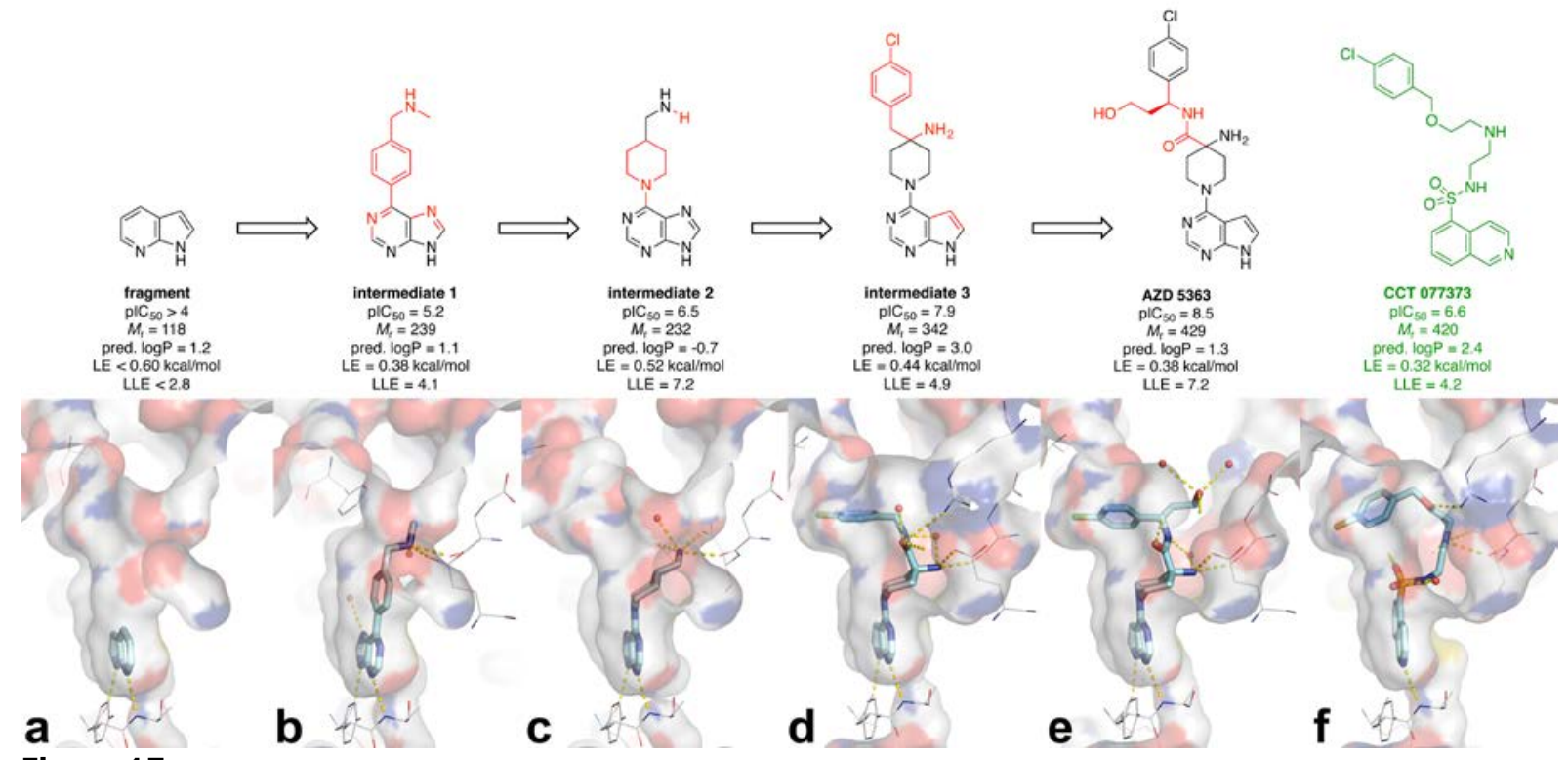

Figure 17 
Table S1. Protein kinase nomenclature.

\section{Name}

Tyrosine-protein kinase ABL1

Tyrosine-protein kinase $A B L 2$

RAC-alpha serine/threonine-protein kinase, protein kinase $B$ alpha

RAC-beta serine/threonine-protein kinase, protein kinase B beta

ALK tyrosine kinase receptor, anaplastic lymphoma kinase

Aurora kinase $A$, aurora 2

Aurora kinase $B$, aurora 1

Aurora kinase $C$, aurora 3

Serine-protein kinase ATM, ataxia telangiectasia mutated

Serine/threonine-protein kinase ATR, ataxia telangiectasia and Rad3-related protein, FRAPrelated protein 1

Tyrosine-protein kinase receptor UFO, AXL oncogene

Serine/threonine-protein kinase A-Raf

Serine/threonine-protein kinase B-Raf

Tyrosine-protein kinase BTK, Bruton tyrosine kinase

Cell division cycle 7-related protein kinase

Cyclin-dependent kinase 1

Cyclin-dependent kinase 2

Cyclin-dependent kinase 4

Cyclin-dependent kinase 6

Cyclin-dependent kinase 7

Cyclin-dependent kinase 9

Serine/threonine-protein kinase Chk1, checkpoint kinase-1

Serine/threonine-protein kinase Chk2, checkpoint kinase-2

Choline kinase alpha

Casein kinase II subunit alpha

RAF proto-oncogene serine/threonine-protein kinase

Proto-oncogene tyrosine-protein kinase Src

Macrophage colony-stimulating factor 1 receptor

DNA-dependent protein kinase catalytic subunit

Epidermal growth factor receptor

Receptor tyrosine-protein kinase erbB-2

Receptor tyrosine-protein kinase erbB-3

Receptor tyrosine-protein kinase erbB-4

Focal adhesion kinase 1

Protein-tyrosine kinase 2-beta, focal adhesion kinase 2

Basic fibroblast growth factor receptor 1, Fms-like tyrosine kinase 2

Fibroblast growth factor receptor 2, Keratinocyte growth factor receptor

Fibroblast growth factor receptor 3

Fibroblast growth factor receptor 4

Receptor-type tyrosine-protein kinase FLT3

Hepatocyte growth factor receptor

\begin{tabular}{|l|l|}
\hline Abbreviation & Synonyms \\
\hline ABL1 & C-Abl \\
\hline ABL2 & ARG \\
\hline AKT1 & PKBa \\
\hline AKT2 & PKB 3 \\
\hline ALK & \\
\hline ARK1 & \\
\hline ARK2 & STK1 \\
\hline ARK3 & \\
\hline ATM & \\
\hline ATR & \\
\hline AXL & \\
\hline A-RAF & PKS \\
\hline B-RAF & \\
\hline BTK & ATK \\
\hline CDC7 & \\
\hline CDK1 & \\
\hline CDK2 & \\
\hline CDK4 & \\
\hline CDK6 & \\
\hline CDK7 & \\
\hline CDK9 & \\
\hline CHK1 & \\
\hline CHK2 & \\
\hline CK & CHETK-a \\
\hline CK IIa & \\
\hline C-RAF & RAF-1 \\
\hline C-SRC & \\
\hline CSF1R & c-Fms \\
\hline DNA-PKCS & DNAPK1, p460 \\
\hline EGFR & ErbB1, HER1 \\
\hline ERBB2 & MLN19, NEU, HER2 \\
\hline ERBB3 & HER3 \\
\hline ERBB4 & HER4 \\
\hline FADK1 & \\
\hline FADK2 & CADTK \\
\hline FGFR1 & bFGF-R-1, FLT1 \\
\hline FGFR2 & K-sam \\
\hline FGFR3 & \\
\hline FGFR4 & \\
\hline FLT3 & \\
\hline HGFR & \\
\hline & \\
\hline
\end{tabular}

\section{Gene}

ABL1 (ABL, JTK7)

ABL2 (ABLL, ARG)

AKT1

AKT2

ALK

AURKA, ARK1, AURA

AURKB, ARK2, STK1

AURKC, ARK3

ATM

ATR, FRP1

$A X L, U F O$

ARAF1 (PKS, PKS2)

BRAF1, RAFB1

BTK, AGMX1, ATK, BPK

CDK1, CDC2, CDKN1

CDK2, CDKN2

CDK4

CDK6, CDKN6

CDK7, CAK, CDKN7, MO15, STK1

CDK9, CDC2L4, TAK

CHEK1, CHK1

CHEK2, CDS1, CHK2, RAD53

CHKA, CHK, CKI

CSNK2A1, CK2A1

RAF1

SRC

CSF1R (FMS)

PRKDC, HYRC, HYRC1

EGFR (ERBB, ERBB1, HER1)

ERBB2 (HER2, MLN19, NEU, NGL)

ERBB3 (HER3)

ERBB4 (HER4)

PTK2, FAK, FAK1

PTK2B, FAK2, PYK2, RAFTK

FGFR1

FGFR2

FGFR3

FGFR4

FLT3 (CD
MET
UniProtKB

P00519

$\mathrm{P} 42684$

P31749

P31751

Q9UM73

014965

Q96GD4

Q9UQB9

Q13315

Q13535

P30530

P10398

P15056

Q06187

000311

$\mathrm{P} 06493$

P24941

$\mathrm{P} 11802$

Q00534

P50613

P50750

014757

096017

P35790

P68400

P04049

P12931

P07333

$\mathrm{P} 78527$

$\mathrm{P} 00533$

P04626

P21860

Q15303

Q05397

Q14289

P11362

P21802

P22607

P22455

P36888

P08581 
Insulin-like growth factor 1 receptor

Insulin receptor

Tyrosine-protein kinase JAK1, Janus kinase 1

Tyrosine-protein kinase JAK2, Janus kinase 2

Tyrosine-protein kinase JAK3, Janus kinase 3

Tyrosine-protein kinase Lck, leukocyte C-terminal Src kinase

Mitogen-activated protein kinase 1 , extracellular signal-regulated kinase 2

Mitogen-activated protein kinase 3, extracellular signal-regulated kinase 1

Dual specificity mitogen-activated protein kinase kinase 1

Dual specificity mitogen-activated protein kinase kinase 2

Mitogen-activated protein kinase 14; Mitogen-activated protein kinase $\mathrm{p} 38-\mathrm{a}$

Maternal embryonic leucine zipper kinase

MAP kinase-interacting serine/threonine-protein kinase 1

MAP kinase-interacting serine/threonine-protein kinase 2

Serine/threonine-protein kinase mTOR

Platelet-derived growth factor receptor alpha

Platelet-derived growth factor receptor beta

3-Phosphoinositide-dependent protein kinase 1

Phosphatidylinositol-4,5-bisphosphate 3-kinase catalytic subunit alpha isoform

Phosphatidylinositol-4,5-bisphosphate 3-kinase catalytic subunit beta isoform

Phosphatidylinositol-4,5-bisphosphate 3-kinase catalytic subunit delta isoform

Phosphatidylinositol-4,5-bisphosphate 3-kinase catalytic subunit gamma isoform

cAMP-dependent protein kinase catalytic subunit alpha

Protein kinase $\mathrm{C}$ beta type

Serine/threonine-protein kinase pim-1

Serine/threonine-protein kinase pim-2

Serine/threonine-protein kinase pim-3

RAC-alpha serine/threonine-protein kinase, protein kinase $B$

Serine/threonine protein kinase PLK1, polo-like kinase 1

Serine/threonine protein kinase PLK4, polo-like kinase 4

Proto-oncogene tyrosine-protein kinase receptor Ret

Rho-associated protein kinase 1

Rho-associated protein kinase 2

Mast/stem cell growth factor receptor Kit

Serine/threonine-protein kinase SMG1

Tyrosine-protein kinase SYK, spleen tyrosine kinase

Angiopoietin-1 receptor; tunica interna endothelial cell kinase

Transforming growth factor-beta receptor type I

High affinity nerve growth factor receptor, tropomyosin-related kinase $\mathrm{A}$

BDNF/NT-3 growth factors receptor, tropomyosin-related kinase B

NT-3 growth factor receptor

Transformation/transcription domain-associated protein

Non-receptor tyrosine-protein kinase TYK2

Vascular endothelial growth factor receptor 1

Vascular endothelial growth factor receptor 2

\begin{tabular}{|c|c|c|c|}
\hline IGF1R & & IGFR1 & P08069 \\
\hline IR & CD220 & ISNR & $\mathrm{P} 06213$ \\
\hline JAK1 & & JAK1 (JAK1A, JAK1B) & P23458 \\
\hline JAK2 & & JAK2 & 060674 \\
\hline JAK3 & LJAK & JAK3 & P52333 \\
\hline LCK & LSK, p56-LCK & LCK & P06239 \\
\hline MAPK1 & $\begin{array}{l}\text { ERK2, MAPK2, p42- } \\
\text { MAPK }\end{array}$ & MAPK1 & P28482 \\
\hline MAPK3 & $\begin{array}{l}\text { ERK1, MAP2K, p44- } \\
\text { MAPK }\end{array}$ & MAPK3 & P27361 \\
\hline MAPKK1 & MKK1, MEK1 & MAP2K1 (MEK1, PRKMK1) & Q02750 \\
\hline MAPKK2 & MEK2 & MAP2K2 (MEK2, MKK2, PRKMK2) & P36507 \\
\hline MAPK14 & p38-a & MAPK14, CSBP, MXI2, SAPK2A & Q16539 \\
\hline MELK & & MELK & Q14680 \\
\hline MNK1 & & MKNK1, MNK1 & Q9BUB5 \\
\hline MNK2 & & MKNK2, GPRK7, MNK2 & Q9HBH9 \\
\hline mTOR & mTOR1 & $\begin{array}{l}\text { MTOR (FRAP, FRAP1，FRAP2， } \\
\text { RAFT1，RAPT1) }\end{array}$ & P42345 \\
\hline PDGFRa & PDGFR2 & PDGFR2 (PDGFR2，RHEPDGFRA) & P16234 \\
\hline PDGFR $\beta$ & PDGFR1 & PDGFRB (PDGFR，PDGFR1) & P09619 \\
\hline PDK1 & PDPK1 & PDPK1 (PDK1) & 015530 \\
\hline PI3Ka & $\mathrm{p} 110 \mathrm{a}$ & PIK3CA & P42336 \\
\hline PI3K $\beta$ & p110ß & PIK3C1 & P42338 \\
\hline PI3Kס & p110ס & PIK3CD & 000329 \\
\hline PI3Ky & p110y & PI3CG & P48736 \\
\hline PKA C-a & & PRKACA & $\mathrm{P} 17612$ \\
\hline$\overline{P K C} \beta$ & & PRKCB, PKCB & P05771 \\
\hline PIM1 & & PIM1 & P11309 \\
\hline PIM2 & & PIM2 & Q9P1W9 \\
\hline PIM3 & & PIM3 & Q86V86 \\
\hline PKB & PKBa, c-Akt & AKT1 (PKB, RAC) & P31749 \\
\hline PLK1 & STPK13 & PLK1, PLK & P53350 \\
\hline PLK4 & SAK & PLK4, SAK & 000444 \\
\hline RET & & & P07949 \\
\hline ROCK-I & p160ROCK & ROCK1 & Q13464 \\
\hline ROCK-II & p164 ROCK-2 & ROCK2 & 075116 \\
\hline SCFR & c-Kit & KIT (SCFR) & P10721 \\
\hline SMG1 & & SMG1，ATX，KIAA0421，LIP & Q96Q15 \\
\hline SYK & & SYK & P43405 \\
\hline TEK & TIE2 & & Q02763 \\
\hline TGFR1 & ALK5, SKR4 & TGFBR1 (ALK5，SRK4) & P36897 \\
\hline TRK1 & TRKA & NTRK1，MTC，TRK，TRKA & P04629 \\
\hline TRK2 & TRKB & NTRK2, TRKB & Q16620 \\
\hline TRK3 & TRKC & NTRK3, TRKC & Q16288 \\
\hline TRRAP & STAF40 & TRRAP, PAF400 & Q9Y4A5 \\
\hline TYK2 & & TYK2 & P29597 \\
\hline VEGFR1 & FLT1, FRT & FLT1 (FLT, FRT, VEGFR1) & P17948 \\
\hline VEGFR2 & KDR, FLK1 & KDR (FLK1，VEGFR2) & P35968 \\
\hline
\end{tabular}


Table S2. BCR-ABL inhibitors.

\begin{tabular}{|l|l|l|l|l|}
\hline Proprietary name & USAN / INN & Corporate code & Sponsor & M \\
\hline Gleevec & Imatinib & STI 571 & Novartis & A \\
\hline Sprycel & Dasatinib & BMS 354825 & BMS & A \\
\hline Tasigna & Nilotinib & AMN 107 & Novartis & A \\
\hline Bosulif & Bosutinib & SKI 606 & Pfizer & Ariad \\
\hline Iclusig & Ponatinib & AP 24534 & II-Yang Pharmaceutical & Jiangsu Hansoh Pharmaceutical \\
\hline Supect & Radotinib & IY 5511 & Novartis & \\
\hline & Flumatinib & & ABL 001 &
\end{tabular}

\section{Main kinase targets}

$A B L$, PDGFR, SCFR, SRC

$A B L$, mutant $A B L, S R C$ (MTKI)

$A B L$, mutant $A B L$, PDGFR, SRC

$A B L$, mutant $A B L, S R C$

$A B L$, mutant $A B L, S R C$, VEGFR, PDGFR, FGFR (MTKI)

$A B L$, mutant $A B L$

$A B L$, mutant $A B L, P D G F R F, S C F R, S R C$ $A B L$

\begin{tabular}{|l|l|}
\hline Development stage & Refs. \\
\hline Approved MAY 2001 & 1 \\
\hline Approved JUN 2006 & 2 \\
\hline Approved OCT 2007 & 3 \\
\hline Approved SEP 2012 & 4 \\
\hline Approved DEC 2012 & 5 \\
\hline Approved OCT 2012 (S. Korea) & 6 \\
\hline Phase-III & 7 \\
\hline Phase-I & 8 \\
\hline
\end{tabular}

Phase-I

Table S3. SRC kinase inhibitors.

\begin{tabular}{|c|c|c|c|c|c|c|}
\hline Proprietary name & USAN / INN & Corporate code & Sponsor & Main kinase targets & Development stage & Refs. \\
\hline & & $\mathrm{KX} 01$ & Thenex \& Hanmi & SRC & Phase-II & 9 \\
\hline & Saracatinib & AZD 0530 & AstraZeneca & $\mathrm{SRC}, \mathrm{ABL}$ & Phase-II & 10 \\
\hline & & AZD 0424 & AstraZeneca & $\mathrm{SRC}, \mathrm{ABL}$ & Phase-I & 11 \\
\hline
\end{tabular}

Table S4. Epidermal growth factor receptor tyrosine kinase inhibitors.

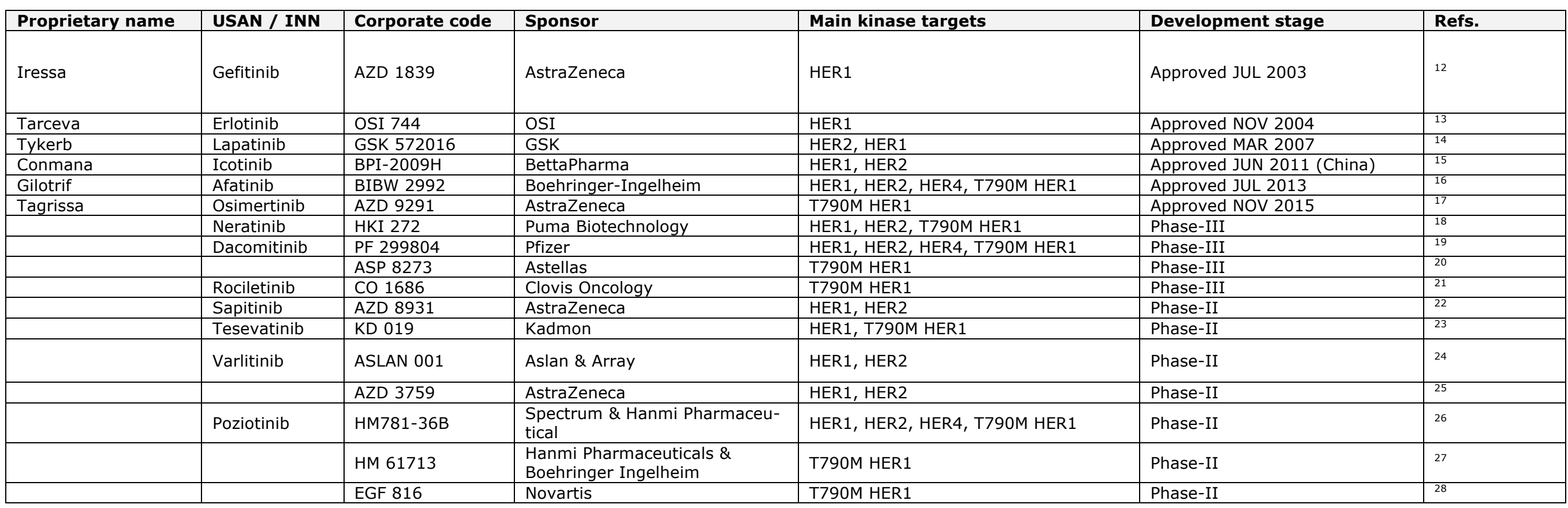




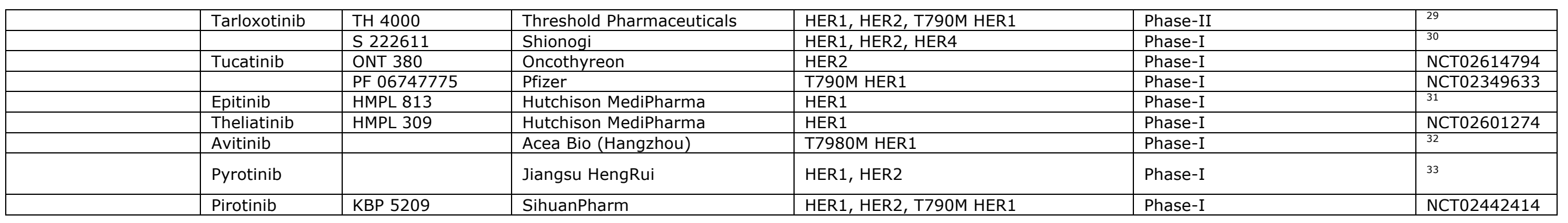

Table S5. Inhibitors of tyrosine kinases in the CSF-1/PDGF receptor subfamily.

\begin{tabular}{|c|c|c|c|c|c|c|}
\hline Proprietary name & USAN / INN & Corporate code & Sponsor & Main kinase targets & Development stage & Refs. \\
\hline Sutent & Sunitinib & SU 11248 & Pfizer & $\begin{array}{l}\text { VEGFR, PDGFR, FLT3, SCFR, RET } \\
\text { (MTKI) }\end{array}$ & Approved JAN 2006 & 34 \\
\hline Votrient & Pazopanib & GW 786034B & GSK & $\begin{array}{l}\text { VEGFR, PDGFR, FGFR, SCFR, FMS } \\
\text { (MTKI) }\end{array}$ & Approved OCT 2010 & 35 \\
\hline Caprelsa & Vandetanib & AZD 6474 & AstraZeneca & $\begin{array}{l}\text { VEGFR, EGFR，PDGFR，FGFR，RET } \\
\text { (MTKI) }\end{array}$ & Approved APR 2011 & 36 \\
\hline Inlyta & Axitinib & AG 013736 & Pfizer & VEGFR，PDGFR，SCFR (MTKI) & Approved JAN 2012 & 37 \\
\hline Stivarga & Regorafenib & BAY 73-4506 & Bayer & $\begin{array}{l}\text { VEGFR, SCFR, RAF, PDGFR, FGFR, KIT } \\
\text { (MTKI) }\end{array}$ & Approved SEP 2012 & 38 \\
\hline Vargatef (Ovef) & Nintedanib & BIBF 1120 & Boehringer-Ingelheim & VEGF, PDGF, FGFR (MTKI) & Approved NOV 2014 & 39 \\
\hline \multirow[t]{2}{*}{ Lenvima } & Lenvatinib & E 7080 & Eisai & $\begin{array}{l}\text { VEGFR, FGFR, PDGFR, SCFR, RET } \\
\text { (MTKI) }\end{array}$ & Approved FEB 2015 & 40 \\
\hline & Midostaurin & PKC 412 & Novartis & FLT3 (MTKI) & Phase-III & 41 \\
\hline \multirow[t]{6}{*}{ Masivet } & Masitinib & $A B 1010$ & AB-Science & KIT, PDGFR, SCFR, FGFR (MTKI) & Phase-III & 42 \\
\hline & Tivozanib & AV 951 & Aveo \& Kyowa Hakko Kirin & VEGFR (MTKI) & Phase-III & 43 \\
\hline & Motesanib & AMG 706 & Amgen \& Millenium-Takeda & VGFR, PDGFR, SCFR (MTKI) & Phase-III & 44 \\
\hline & Crenolanib & CP 868,596 & Arog & PDGFR, SCFR, FLT3 (selective) & Phase-III & 45 \\
\hline & Fruquintinib & HMPL 013 & Hutchison MediPharma & VEGFR (selective) & Phase-III & 46 \\
\hline & Quizartinib & AC 220 & Daiichi & FLT3 (selective) & Phase-III & 47 \\
\hline \multirow[t]{15}{*}{ Recentin } & Cediranib & AZD 2171 & AstraZeneca & VEGFR, PDGFR (selective) & Phase-III & 48 \\
\hline & Pexidartinib & PLX 3397 & Daiichi \& Plexxikon & CSF1R，SCFR，FLT3 (selective) & Phase-III & 49 \\
\hline & Sulfatinib & HMPL 012 & Hutchison MediPharma & VEGFR, FGFR & Phase-III & NCT02589821 \\
\hline & Apatinib & YN 968D1 & LSK BioPharma & VEGF & Phase-II & 50 \\
\hline & Lucitanib & E 3810 & Clovis Oncology \& Servier & VEGFR, CSF1R, FGFR (selective) & Phase-II & 51 \\
\hline & & $\times 82$ & Tyrogenex & VEGFR, PDGFR & Phase-II & NCT02146222 \\
\hline & Anlotinib & AL 3818 & Advenchen & VEGFR, FGFR & Phase-II & NCT02649361 \\
\hline & & $\mathrm{AKN}-028$ & Akinion & FLT3 (selective) & Phase-II & 52 \\
\hline & Linifanib & ABT 869 & Abbott & VEGFR, FLT3 (MTKI) & Phase-I & 53 \\
\hline & & PLX 9486 & Plexxikon & KIT & Phase-I & NCT02401815 \\
\hline & & PLX 7486 & Plexxikon \& Daiichi Sankyo & FLT3, pan-TRK & Phase-I & NCT01804530 \\
\hline & & ARRY 382 & Array & CSF1R & Phase-I & 54 \\
\hline & Dovitinib & TKI 258 & Novartis & VEGFR，FLT3，FGFR (MTKI) & Phase-I & 55 \\
\hline & Sitravatinib & MGCD 516 & Mirati & MTKI & Phase-I & 56 \\
\hline & Tafetinib & SIM 010603 & Nanjing Yoko Biomedical & MTKI & Phase-I & 57 \\
\hline
\end{tabular}


Table S6. Fibroblast growth factor receptor kinase inhibitors.

\begin{tabular}{|c|c|c|c|c|c|c|}
\hline Proprietary name & USAN / INN & Corporate code & Sponsor & Main kinase targets & Development stage & Refs. \\
\hline & & AZD 4547 & AstraZeneca & Pan-FGFR & Phase-II & \\
\hline & Infigratinib & BGJ 398 & Novartis & pan-FGFR & Phase-II & 59 \\
\hline & Erdafitinib & JNJ 42756493 & Jansen & pan-FGFR & Phase-II & 60 \\
\hline & & E 7090 & Eisai & pan-FGFR & Phase-I & 61 \\
\hline & & BAY 1163877 & Bayer & pan-FGFR & Phase-I & 62 \\
\hline & & ARQ 087 & ArQule & FGFR & Phase-I & 63 \\
\hline & & INCB 054828 & Incyte & FGFR & Phase-I & 64 \\
\hline & & PRN 1371 & Principia Biopharma & FGFR & Phase-I & 65 \\
\hline & & FGF 401 & Novartis & FGFR4 & Phase-I & NCT02325739 \\
\hline & & TAS 120 & Taiho Pharmaceutical & pan-FGFR & Phase-I & 66 \\
\hline
\end{tabular}

Table S7. Hepatocyte growth factor receptor kinase (MET) inhibitors.

\begin{tabular}{|c|c|c|c|c|c|c|}
\hline Proprietary name & USAN / INN & Corporate code & Sponsor & Main kinase targets & Development stage & Refs. \\
\hline \multirow{10}{*}{ Cometriq } & Cabozantinib & $\mathrm{XL} 184$ & Exelixis & MET, RET, VEGFR & Approved NOV 2012 & 67 \\
\hline & Tivantinib & ARQ 197 & ArQule \& Daiichi & MET & Phase-III & 68 \\
\hline & Foretinib & GSK 1363089 & GSK \& Exelixis & MET, VEGFR & Phase-II & 69 \\
\hline & & ASLAN 002 & Aslan & MET & Phase-II & 70 \\
\hline & Savolitinib & AZD 6094 & $\begin{array}{l}\text { AstraZeneca \& Hutchison Me- } \\
\text { diPharma }\end{array}$ & MET & Phase-II & 71 \\
\hline & Tepotinib & EMD 1214063 & Merck Serono & MET & Phase-II & 72 \\
\hline & & AMG 337 & Amgen & MET & Phase-II & 73 \\
\hline & Merestinib & LY 2801653 & Eli Lilly & MET (MTKI) & Phase-II & 75 \\
\hline & Altiratinib & DCC 2701 & Deciphera & MET (MTKI) & Phase-I & 76 \\
\hline & & SAR 125844 & Sanofi & MET & Phase-I & 77 \\
\hline
\end{tabular}

Table S8. ALK tyrosine kinase receptor inhibitors.

\begin{tabular}{|c|c|c|c|c|c|c|}
\hline Proprietary name & USAN / INN & Corporate code & Sponsor & Main kinase targets & Development stage & Refs. \\
\hline Xalkori & Crizotinib & PF 02341066 & Pfizer & ALK (c-MET) & Approved AUG 2011 & \\
\hline Zykadia & Ceritinib & LDK 378 & Novartis & ALK & Approved APR 2014 & 79 \\
\hline \multirow[t]{3}{*}{ Alecensa } & Alectinib & AF-802 & Roche & ALK & Approved DEC 2015 & 80 \\
\hline & Brigatinib & AP 26113 & Ariad & ALK (HER) & Phase-III & 81 \\
\hline & Belizatinib & TSR 011 & Tesaro & ALK & Phase-II & NCT02048488 \\
\hline
\end{tabular}

Table S9. TRK inhibitors.

\begin{tabular}{|c|c|c|c|c|c|c|}
\hline Proprietary name & USAN / INN & Corporate code & Sponsor & Main kinase targets & Development stage & Refs. \\
\hline & Entrectinib & RXDX 101 & $\begin{array}{l}\text { Ignyta \& Nerviano Medical } \\
\text { Sciences }\end{array}$ & Pan-TRK (ROS1, ALK) & Phase-II & 83 \\
\hline & & LOXO 101 & Loxo Oncology \& Array & Pan-TRK & Phase-II & 84 \\
\hline & & DS 6051b & Daiichi Sankyo & Pan-TRK (ROS1) & Phase-I & NCT02279433 \\
\hline
\end{tabular}


Table S10. AXL inhibitors.

\begin{tabular}{|l|l|l|}
\hline Proprietary name & USAN / INN & Corporate code \\
\hline & Gilteritinib & ASP 2215 \\
\hline & & BGB 324 \\
\hline
\end{tabular}

$A X L, F L T 3$

Table S11. TGFR kinase inhibitors.

\begin{tabular}{|l|l|l|}
\hline Proprietary name & USAN / INN & Corporate code \\
\hline & Galunisertib & LY2157299 \\
\hline & & TEW 7197 \\
\hline
\end{tabular}

Sponsor

Eli Lilly

MedPacto

\section{Sponsor}

Astellas

BerGenBio
Main kinase targets

TGFR1

TGFR1
Development stage

Phase-III

Phase-I

Development stage

Phase-II

Phase-I

\section{Main kinase targets}

JAK2

\begin{tabular}{l|l} 
Corporate code & Sponsor \\
\hline INC 424 & Novartis \& Incyte
\end{tabular}

CTI BioPharma

SB 1518

NS-018

CYT 387

PRT 062070

INCB 052793

INCB 039110
Nippon Shinyaku

Gilead

Portola

Incyte

Incyte
KK2 (FLT3)

JAK2

JAK1, JAK2

JAK (SYK)

JAK1

JAK1
Development stage

Approved NOV 201

Phase-III

Phase-II

Phase-II

Phase-II

Phase-I

Phase-I

Refs.

(2)

Table S13. Bruton kinase BTK inhibitors.

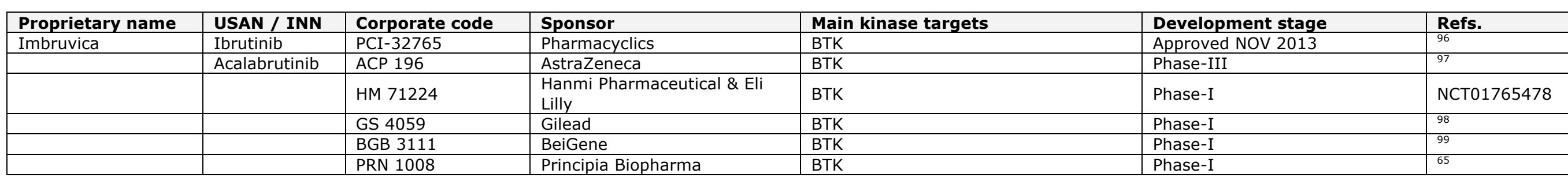

Table S14. Focal adhesion kinase 1 inhibitors.

\begin{tabular}{|c|c|c|c|c|c|c|}
\hline Proprietary name & USAN / INN & Corporate code & Sponsor & Main kinase targets & Development stage & Refs. \\
\hline & & GSK 2256098 & GSK & FAK & Phase-II & 100 \\
\hline & Defactinib & VS 6063 & Verastem & FAK, PYK2 & Phase-II & 101 \\
\hline & & VS 4718 & Verastem & FAK & Phase-I & 102 \\
\hline
\end{tabular}

Table S15. SYK inhibitors.

\begin{tabular}{|l|l|l|}
\hline Proprietary name & USAN / INN & Corporate code \\
\hline & Entospletinib & GS-9973 \\
\hline & & TAK 659 \\
\hline
\end{tabular}

\begin{tabular}{l} 
Sponsor \\
Gilead \\
\hline Millenium
\end{tabular}

Millenium

Main kinase targets

SYK

Table S16. Phosphatidylinositol-3-kinase pathway inhibitors

\begin{tabular}{|l|l|l|l|}
\hline Proprietary name & USAN / INN & Corporate code & Sponsor \\
\hline Torisel & Temsirolimus & CCI 779 & Pfizer \\
\hline
\end{tabular}
Pfizer

\section{Main kinase targets} mTOR

\section{Development stage}

Phase-II

Phase-I 


\begin{tabular}{|c|c|c|c|c|c|c|}
\hline Afinitor & Everolimus & RAD001 & Novartis & mTOR & Approved MAR 2009 & 106 \\
\hline \multirow[t]{25}{*}{ Zydelig } & Idelalisib & GS 1101 & Gilead & PI3K $\delta$ & Approved JUL 2014 & 107 \\
\hline & Duvelisib & INK 1197 & Infinity \& AbbVie & PI3Kס, PI3KY & Phase-III & 108 \\
\hline & Buparlisib & BKM 120 & Novartis & Pan-PI3K & Phase-III & 109 \\
\hline & Alpelisib & BYL 719 & Novartis & $\mathrm{PI} 3 \mathrm{Ka}$ & Phase-III & 110 \\
\hline & Taselisib & GDC 0032 & Genentech & $\mathrm{PI} 3 \mathrm{Ka}$ & Phase-III & 112 \\
\hline & Voxtalisib & XL 765 & Exelixis, Sanofi & Pan-PI3K, mTOR & Phase-II & 113 \\
\hline & Vistusertib & AZD 2014 & AstraZeneca & mTOR & Phase-II & 114 \\
\hline & & SF 1126 & SignalRX Pharmaceuticals & PI3K (MTKI) & Phase-II & 115 \\
\hline & Sapanisertib & MLN 0128 & Millenium & mTOR & Phase-II & 116 \\
\hline & & AMG 319 & Amgen & PI3Kס & Phase-II & 117 \\
\hline & & CC 223 & Celgene & mTOR & Phase-II & 118 \\
\hline & & CC 115 & Celgene & mTOR (DNA-PK) & Phase-II & 119 \\
\hline & & GSK 2636771 & GSK & PI3K $\beta$ & Phase-II & 120 \\
\hline & & CUDC 907 & Curis & PI3K (HDAC) & Phase-II & 121 \\
\hline & & LY 3023414 & Eli Lilly & Pan-PI3K, mTOR & Phase-II & 122 \\
\hline & & PQR 309 & Piqur & mTOR, PI3K & Phase-II & NCT02723877 \\
\hline & Pictilisib & GDC-0941 & Genentech & PI3K & Phase-I & 123 \\
\hline & Gedatolisib & PF-05212384 & Pfizer & PI3K, mTOR & Phase-I & 124 \\
\hline & & ZSTK 474 & Zenyaku Kogyo & PI3K & Phase-I & 125 \\
\hline & & AZD 8835 & AstraZeneca & PI3Ka, PI3Kס & Phase-I & 127 \\
\hline & & HMPL 689 & Hutchison MediPharma & PI3Kס & Phase-I & NCT02631642 \\
\hline & & INCB 050465 & Incyte & PI3Kס & Phase-I & 128 \\
\hline & & VS 5584 & Verastem & Pan-PI3K, mTOR & Phase-I & 129 \\
\hline & & KA 2237 & Karus Therapeutics & PI3K $\beta$, PI3Kס & Phase-I & NCT02679196 \\
\hline & & CDZ 173 & Novartis & PI3Kס & Phase-I & NCT02435173 \\
\hline
\end{tabular}

Table S17. RAC-alpha serine/threonine-protein kinase, protein kinase B inhibitors.

\begin{tabular}{|c|c|c|c|c|c|c|}
\hline Proprietary name & USAN / INN & Corporate code & Sponsor & Main kinase targets & Development stage & Refs. \\
\hline & & AZD 5363 & AstraZeneca \& Astex & AKT & Phase-II & 130 \\
\hline & Ipatasertib & GDC 0068 & Genentech \& Array & AKT & Phase-II & 131 \\
\hline & & LY 2780301 & Lilly & AKT (p70S6) & Phase-II & 132 \\
\hline & Afuresertib & ASB 183 & Novartis & Pan-AKT & Phase-II & 133 \\
\hline & & ARQ 092 & ArQule & AKT & Phase-I & 134 \\
\hline & & MK 2206 & Merck & AKT & Phase-I & 135 \\
\hline & & M 2698 & Merck Serono & AKT (p70S6) & Phase-I & NCT01971515 \\
\hline & & AT 13148 & Astex & AKT (MTKI) & Phase-I & 136 \\
\hline & & BAY 1125976 & Bayer & AKT1, AKT2 & Phase-I & 137 \\
\hline
\end{tabular}

Table S18. PIM inhibitors.

\begin{tabular}{|l|l|l|l|}
\hline Proprietary name & USAN / INN & Corporate code & Sponsor \\
\hline & & PIM 447 (LGH 447) & Novartis \\
\hline & & INCB 053914 & Incyte \\
\hline
\end{tabular}

Main kinase targets

Pan-PIM

PIM
Development stage

Phase-I

Phase-II
Refs.

139 
Table S19. RAF kinase inhibitors.

\begin{tabular}{|c|c|c|c|c|c|c|}
\hline Proprietary name & USAN / INN & Corporate code & Sponsor & Main kinase targets & Development stage & Refs. \\
\hline Nexavar & Sorafenib & BAY 43-9006 & Bayer \& Onyx & RAF (MTKI) & Approved DEC 2005 & \\
\hline Zelboraf & Vemurafenib & PLX 4032 & Plexxikon \& Roche & Mutant BRAF & Approved AUG 2011 & 141 \\
\hline \multirow[t]{8}{*}{ Tafinlar } & Dabrafenib & GSK 2118436 & GSK & ARAF, BRAF, CRAF & Approved MAY 2013 & 142 \\
\hline & Encorafenib & LGX 818 & Novartis \& Array & Mutant RAF & Phase-III & 143 \\
\hline & & PLX 8394 & Plexxikon \& Daiichi Sankyo & Mutant B-RAF & Phase-II & 144 \\
\hline & & RXDX 105 & Ignyta & RAF (RET) & Phase-I & 145 \\
\hline & & MLN 2480 & Millenium & RAF & Phase-I & 146 \\
\hline & & HM 95573 & Hanmi Pharmaceutical & Pan-RAF & Phase-I & NCT02405065 \\
\hline & & LY 3009120 & Eli Lilly & Pan-RAF & Phase-I & 147 \\
\hline & & BGB 283 & Merck Serono \& BeiGene & Pan-RAF & Phase-I & 148 \\
\hline
\end{tabular}

Table S20. Mitogen-activated protein kinase kinase kinase (MEK) inhibitors.

\begin{tabular}{|c|c|c|c|c|c|c|}
\hline Proprietary name & USAN / INN & Corporate code & Sponsor & Main kinase targets & Development stage & Refs. \\
\hline Mekinist & Trametinib & GSK 1120212 & GSK & MEK1, MEK2 & Approved MAY 2013 & 149 \\
\hline \multirow{8}{*}{ Cotellic } & Cobimetinib & GDC 0973 & Genentech \& Exelixis & MEK & Approved NOV 2015 & 150 \\
\hline & Selumetinib & AZD 6244 & Array \& AZ & MEK1, MEK2 (AKT) & Phase-III & 151,152 \\
\hline & Binimetinib & MEK 162 & Novartis \& Array & MEK1, MEK2 & Phase-III & 153 \\
\hline & Pimasertib & AS703026 & Merck Serono & MEK & Phase-II & 155 \\
\hline & & PD 325901 & Pfizer & MEK & Phase-II & 156 \\
\hline & & TAK 733 & Millenium-Takeda & MEK & Phase-I & 157 \\
\hline & & RG 7304 & Roche & MEK1 (pan-RAF) & Phase-I & 158 \\
\hline & & E 6201 & Strategia Therapeutics & MEK & Phase-I & 159 \\
\hline
\end{tabular}

Table S21. ERK inhibitors.

\begin{tabular}{|c|c|c|c|c|c|c|}
\hline Proprietary name & USAN / INN & Corporate code & Sponsor & Main kinase targets & Development stage & Refs. \\
\hline & Ulixertinib & BVD 523 & BioMed Valley Discoveries & ERK1, ERK2 & Phase-II & \\
\hline & & GDC 0994 & Genentech \& Array & ERK1，ERK2 & Phase-I & 161 \\
\hline & & CC 90003 & Celgene & ERK & Phase-I & NCT02313012 \\
\hline
\end{tabular}

Table S22. p38 MAP kinase inhibitors.

\begin{tabular}{|c|c|c|c|c|c|c|}
\hline Proprietary name & USAN / INN & Corporate code & Sponsor & Main kinase targets & Development stage & Refs. \\
\hline & Ralimetinib & LY 2228820 & Lilly & p38 MAPK & Phase-II & \\
\hline & Pexmetinib & ARRY-614 & Array & p38 MAPK & Phase-I & 163 \\
\hline
\end{tabular}

Table S23. Cyclin-dependent kinase inhibitors.

\begin{tabular}{|c|c|c|c|c|c|c|}
\hline Proprietary name & USAN / INN & Corporate code & Sponsor & Main kinase targets & Development stage & Refs. \\
\hline \multirow{6}{*}{ Ibrane } & Palbociclib & PD 332991 & Pfizer & CDK4, CDK 6 & Approved FEB 2015 & 164 \\
\hline & Ribociclib & LEE 011 & Astex \& Novartis & CDK4, CDK6 & Phase-III & 165 \\
\hline & Abemaciclib & LY 2835219 & Eli Lilly & CDK4, CDK6 & Phase-III & 166 \\
\hline & Dinaciclib & $\mathrm{SCH} 727965$ & Merck & CDK1, CDK2, CDK5, CDK9 & Phase-II & 167 \\
\hline & & AT 7519 & Astex & Pan-CDK & Phase-II & 168 \\
\hline & Milciclib & PHA 848125 & $\begin{array}{l}\text { Nerviano Medical Sciences \& } \\
\text { Tiziana Life Sciences }\end{array}$ & CDK1, CDK2, CDK4 (TRKA) & Phase-II & 169 \\
\hline
\end{tabular}




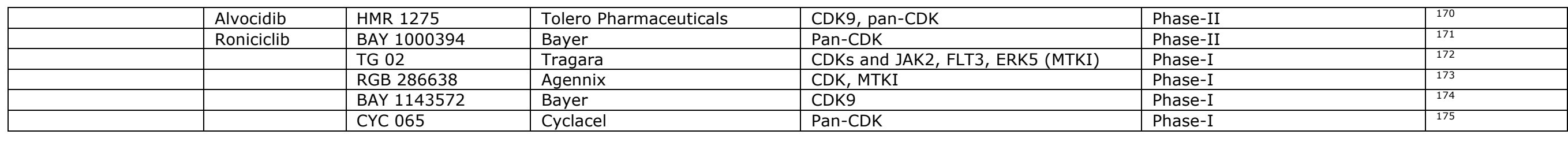

Table S24. Aurora kinase inhibitors.

\begin{tabular}{|c|c|c|c|c|c|c|}
\hline Proprietary name & USAN / INN & Corporate code & Sponsor & Main kinase targets & Development stage & Refs. \\
\hline & Alisertib & MLN 8237 & Millenium-Takeda & ARK-A & Phase-III & 176 \\
\hline & & ENMD 2076 & CASI Pharmaceuticals & ARK (MTKI) & Phase-II & 177 \\
\hline & Ilorasertib & ABT 348 & AbbVie & ARK (MTKI) & Phase-II & 178 \\
\hline & Danusertib & PHA 739358 & Nerviano Medical Sciences & Pan-ARK (ABL) & Phase-II & 179 \\
\hline & Barasertib & AZD 2811 & $\begin{array}{l}\text { Bind Therapeutics \& Astra- } \\
\text { Zeneca }\end{array}$ & ARK-B & Phase-I & 180 \\
\hline & & TAS 119 & Taiho Pharma & ARK-A & Phase-I & 181 \\
\hline
\end{tabular}

Table S25. Polo-like kinase inhibitors.

\begin{tabular}{|c|c|c|c|c|c|c|}
\hline Proprietary name & USAN / INN & Corporate code & Sponsor & Main kinase target & Development stage & Refs. \\
\hline & Volasertib & BI 6727 & Boehringer-Ingelheim & PLK1 & Phase-III & \\
\hline & & CFI 400945 & $\begin{array}{l}\text { University Health Network, } \\
\text { Toronto }\end{array}$ & PLK4 & Phase-I & 183 \\
\hline
\end{tabular}

Table S26. Checkpoint kinase inhibitors.

\begin{tabular}{|c|c|c|c|c|c|c|}
\hline Proprietary name & USAN / INN & Corporate code & Sponsor & Main kinase targets & Development stage & Refs. \\
\hline & Prexasertib & LY 2606368 & Eli Lilly \& Array & CHK1 & Phase-II & 184 \\
\hline & & GDC 0575 & Genentech & CHK1 & Phase-I & 185 \\
\hline
\end{tabular}

Table S27. ATR inhibitors.

\begin{tabular}{|c|c|c|c|c|c|c|}
\hline Proprietary name & USAN / INN & Corporate code & Sponsor & Main kinase targets & Development stage & Refs. \\
\hline & & VX 970 & Vertex & ATR & Phase-II & \\
\hline & & VX 803 & Vertex & ATR & Phase-I & NCT02278250 \\
\hline & & AZD 6738 & AstraZeneca & ATR & Phase-I & \\
\hline
\end{tabular}

Table S28. DNAPK inhibitors.

\begin{tabular}{|c|c|c|c|c|c|c|}
\hline Proprietary name & USAN / INN & Corporate code & Sponsor & Main kinase targets & Development stage & Refs. \\
\hline & & VX 984 & Vertex & DNAPK & Phase-I & NCT02644278 \\
\hline & & M 3814 & Merck Serono & DNAPK & Phase-I & NCT02316197 \\
\hline
\end{tabular}

Table S29. New first-in-class kinase inhibitors.

\begin{tabular}{|c|c|c|c|c|c|c|}
\hline Proprietary name & USAN / INN & Corporate code & Sponsor & Main kinase targets & Development stage & Refs. \\
\hline & Linsitinib & OSI 906 & Astellas & IGF-1R \& IR & Phase-III & 188 \\
\hline & & AZD 1775 & AstraZeneca & WEE1 & Phase-II & 189 \\
\hline & Silmitasertib & CX 4945 & Senhwa Biosciences & CK2 & Phase-II & 190 \\
\hline & Amcasertib & BBI 503 & Boston Biomedical & Cancer stem cell pathway kinases & Phase-II & 191 \\
\hline
\end{tabular}




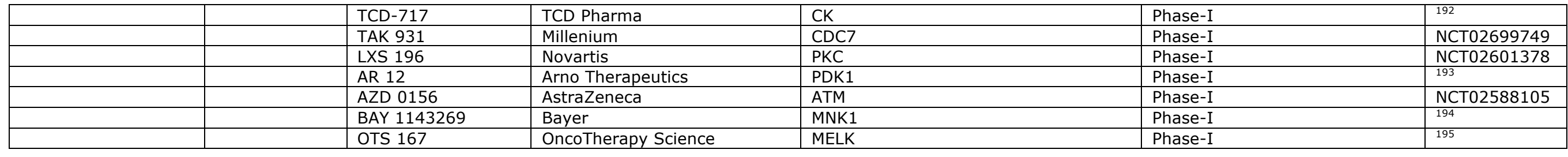

\section{References}

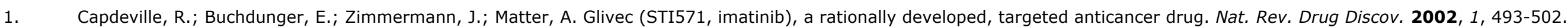

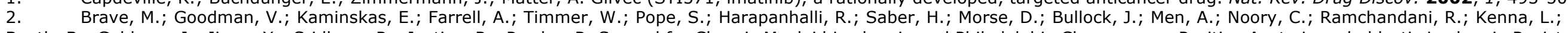

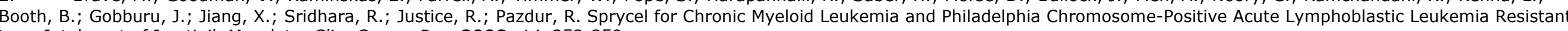
to or Intolerant of Imatinib Mesylate. Clin. Cancer Res. 2008, 14, 352-359.

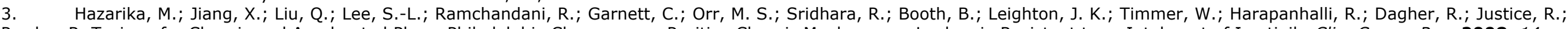

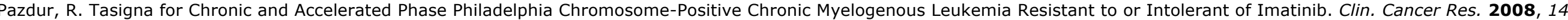
5325-5331.

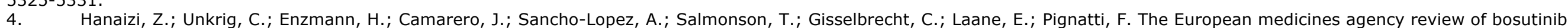

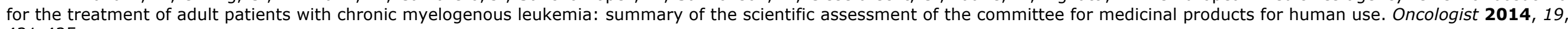
$421-425$.

5. Frankfurt, O.; Licht, J. D. Ponatinib-A Step Forward in Overcoming Resistance in Chronic Myeloid Leukemia. Clin. Cancer Res. 2013, 19, 5828-5834.

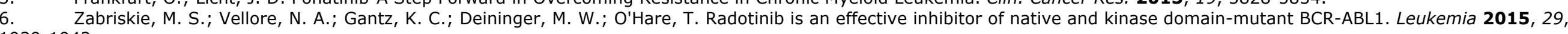
1939-1942.

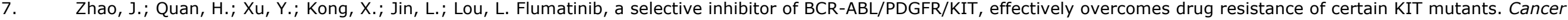
Sci. 2014, 105, 117-125.

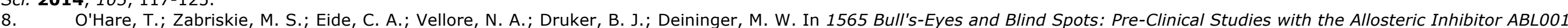
in BCR-ABL1 Compound Mutation-Based Resistance, ASH 57th Annual Meeting, Orlando, FL, USA, 2015; Orlando, FL, USA, 2015; $\mathrm{p} \mathrm{Abs.} \mathrm{1565.}$

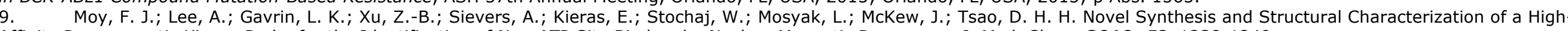
Affinity Paramagnetic Kinase Probe for the Identification of Non-ATP Site Binders by Nuclear Magnetic Resonance. J. Med. Chem. 2010, 53, 1238-1249.

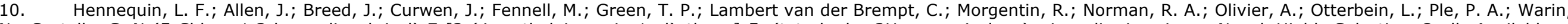

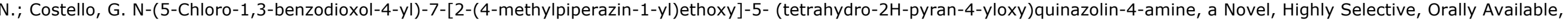
Dual-Specific c-Src/Abl Kinase Inhibitor. J. Med. Chem. 2006, 49, 6465-6488.

11. Roskoski, R. Src protein-tyrosine kinase structure, mechanism, and small molecule inhibitors. Pharmacol. Res. 2015, 94, 9-25,

11. Cohen, M. H.; Williams, G. A.; Sridhara, R.; Chen, G.; Pazdur, R. FDA drug approval summary: gefitinib (ZD1839) (Iressa) tablets. Oncologist 2003, 8, 303-306.

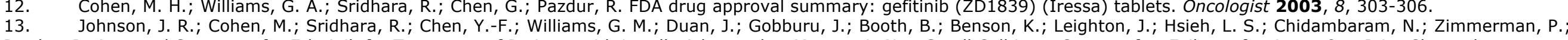

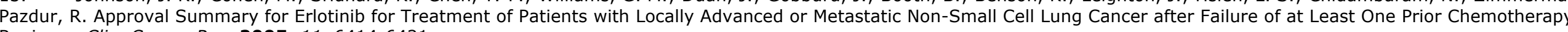
Regimen. Clin. Cancer Res. 2005, 11, 6414-6421.

14. Moy, B.; Kirkpatrick, P.; Kar, S.; Goss, P. Lapatinib. Nat. Rev. Drug Discovery 2007, 6, 431-432.

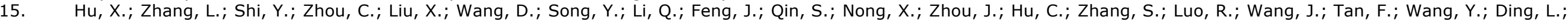

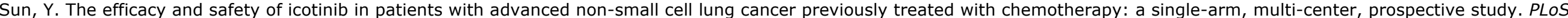
ONE $2015,10,00142500$.

16. Dungo, R. T.; Keating, G. M. Afatinib: First Global Approval. Drugs 2013, 73, 1503-1515.

17. Greig, S. L. Osimertinib: First Global Approval. Drugs 2016, 76, 263-273.

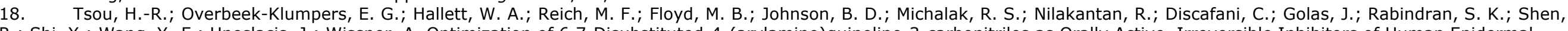

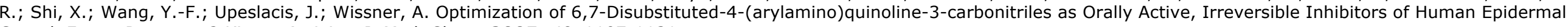
Growth Factor Receptor-2 Kinase Activity. J. Med. Chem. 2005, 48, 1107-1131.

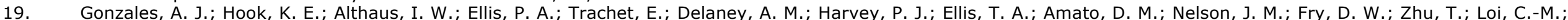

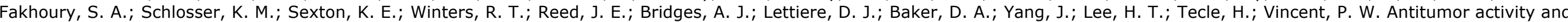
pharmacokinetic properties of PF-00299804, a second-generation irreversible pan-erbB receptor tyrosine kinase inhibitor. Mol. Cancer Ther. 2008, $7,1880-1889$.

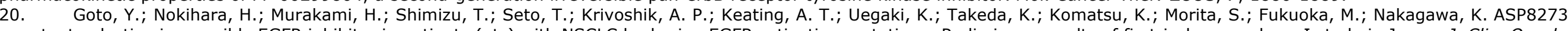

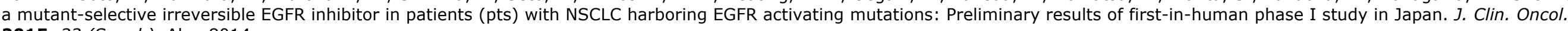
2015, 33 (Suppl.), Abs. 8014. 


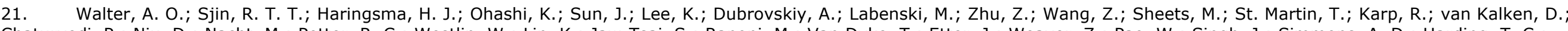

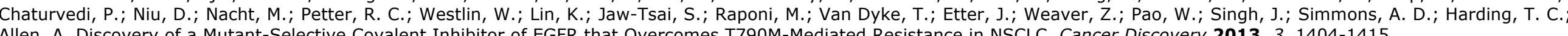
Allen, A. Discovery of a Mutant-Selective Covalent Inhibitor of EGFR that Overcomes T790M-Mediated Resistance in NSCLC. Cancer Discovery 2013, 3, 1404-1415.

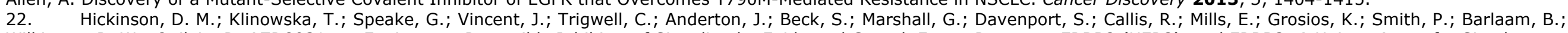

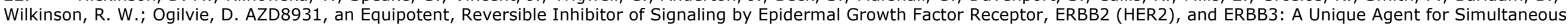
ERBB Receptor Blockade in Cancer. Clin. Cancer Res. 2010, 16, 1159-1169.

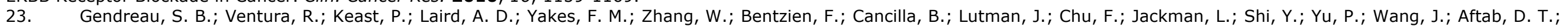

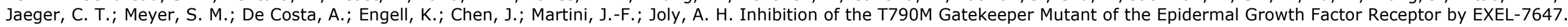
Clin. Cancer Res. 2007, 13, 3713-3723.

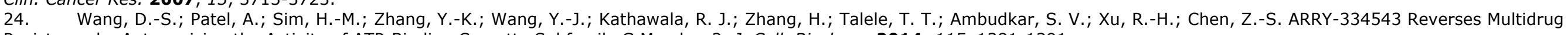
Resistance by Antagonizing the Activity of ATP-Binding Cassette Subfamily G Member 2. J. Cell. Biochem. 2014, 115, 1381-1391.

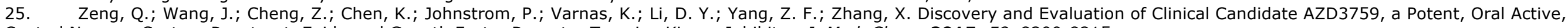
Central Nervous System-Penetrant, Epidermal Growth Factor Receptor Tyrosine Kinase Inhibitor. J. Med. Chem. 2015, 58, 8200-8215.

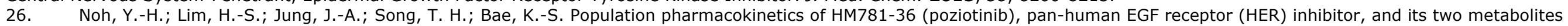
in patients with advanced solid malignancies. Cancer Chemother. Pharmacol. 2015, 75, 97-109.

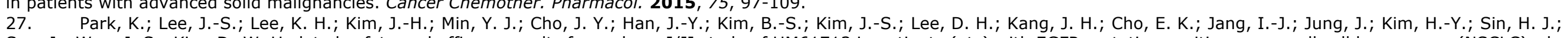

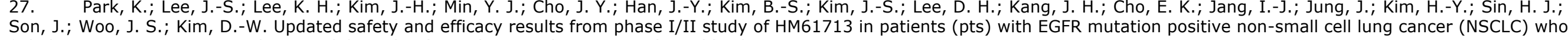
failed previous EGFR-tyrosine kinase inhibitor (TKI). J. Clin. Oncol. 2015, 33 (Suppl.), Abs. 8084

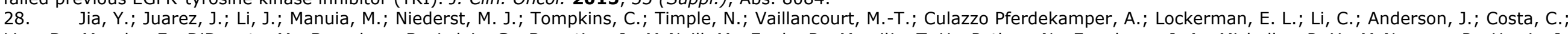

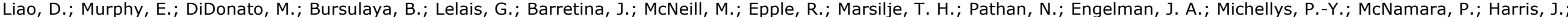

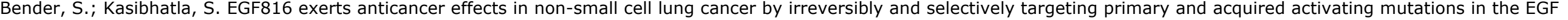

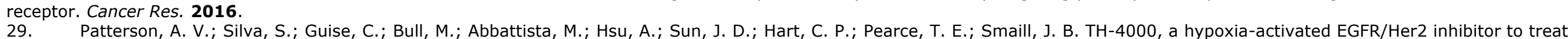
29. Patterson, A. V.; Silva, S.; Guise, C.; Bull, M.; Abbattista, M.; Hsu, A.; Sun, J. D.;
EGFR-TKI resistant T790M-negative NSCLC. J. Clin. Oncol. 2016, 33 (Suppl.), Abs. E13548.

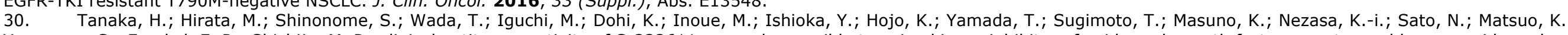

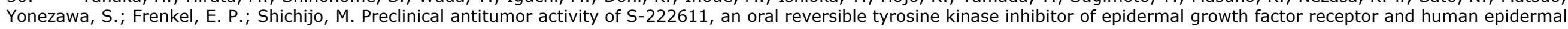
growth factor receptor 2. Cancer Sci. 2014, 105, 1040-1048.

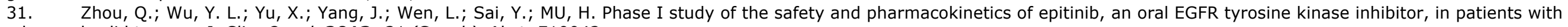
advanced solid tumors. J. Clin. Oncol. 2013, 31 (Suppl.), Abst. E19042.

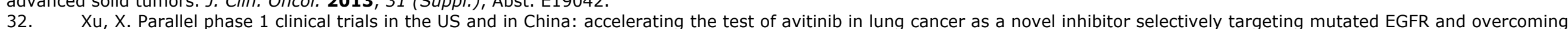

32. Xu, X. Parallel phase 1 clinical trials in the US and in Chin
T790M-induced resistance. Chin. J. Cancer 2015, 34, 285-287.

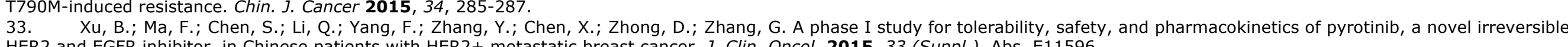
HER2 and EGFR inhibitor, in Chinese patients with HER2+ metastatic breast cancer. J. Clin. Oncol. 2015, 33 (Suppl.), Abs. E11596.

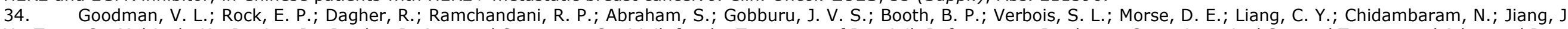

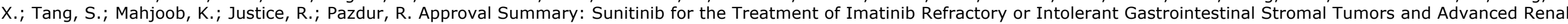
Cell Carcinoma. Clin. Cancer Res. 2007, 13, 1367-1373.

35. Keisner, S. V.; Shah, S. R. Pazopanib: the newest tyrosine kinase inhibitor for the treatment of advance or metastatic renal cell carcinoma. Drugs 2011, $71,443-454$.

36. Chau, N. G.; Haddad, R. I. Vandetanib for the Treatment of Medullary Thyroid Cancer. Clin. Cancer Res. 2013, 19, 524-529.

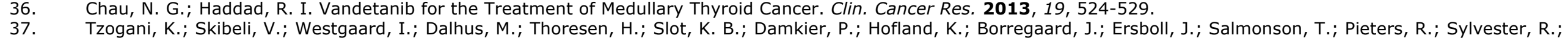

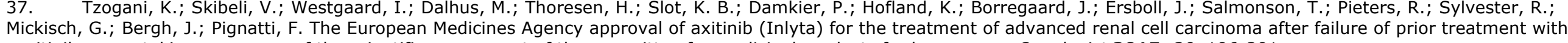
sunitinib or a cytokine: summary of the scientific assessment of the committee for medicinal products for human use. Oncologist 2015, $20,196-201$.

38. Carter, N. J. Regorafenib: A Review of Its Use in Previously Treated Patients with Progressive Metastatic Colorectal Cancer. Drugs Aging 2014, 31, 67-78.

39. McCormack, P. L. Nintedanib: First Global Approval. Drugs 2015, 75, 129-139.

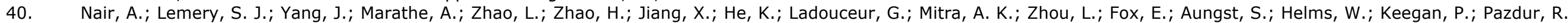
FDA Approval Summary: Lenvatinib for Progressive, Radio-iodine-Refractory Differentiated Thyroid Cancer. Clin. Cancer Res. 2015, $21,5205-5208$.

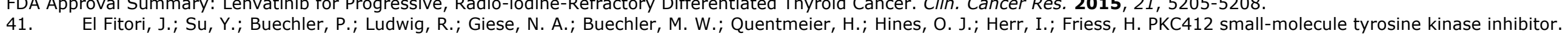

41. El Fitori, J.; Su, Y.; Buechler, P.; Ludwig, R.; Giese, N. A.; Buechler, M. W.
Single-compound therapy for pancreatic cancer. Cancer 2007, 110, 1457-1468.

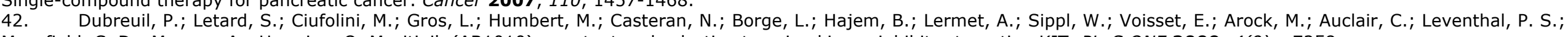

Mansfield, C. D.; Moussy, A.; Hermine, O. Masitinib (AB1010), a potent and selective tyrosine kinase inhibitor targeting KIT. PLoS ONE 2009, 4(9), e7258.

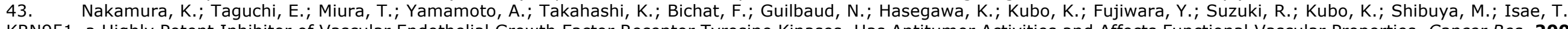

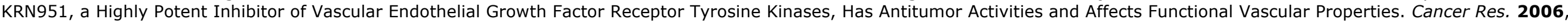
$66,9134-9142$. 


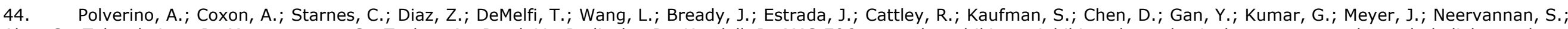

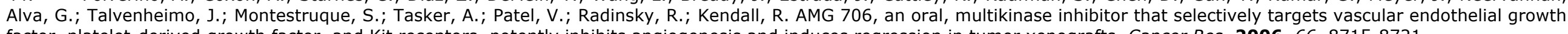
factor, platelet-derived growth factor, and Kit receptors, potently inhibits angiogenesis and induces regression in tumor xenografts. Cancer Res. 2006, 66, 8715-8721.

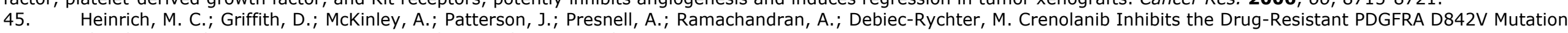

Associated with Imatinib-Resistant Gastrointestinal Stromal Tumors. Clin. Cancer Res. 2012, 18, 4375-4384.

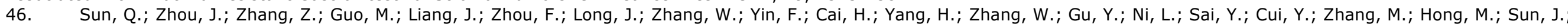

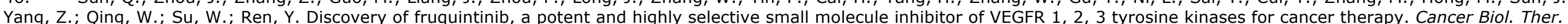
Yang, Z.; Qing, W.; Su,

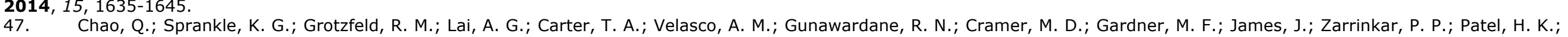

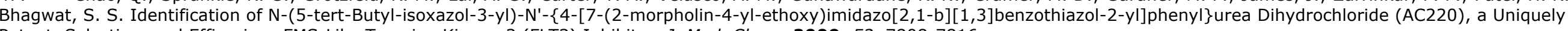
Potent, Selective, and Efficacious FMS-Like Tyrosine Kinase-3 (FLT3) Inhibitor. J. Med. Chem. 2009, 52, 7808-7816.

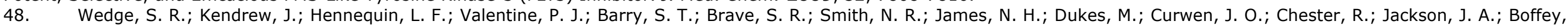

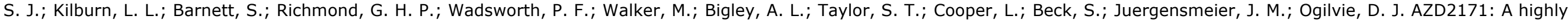
potent, orally bioavailable, vascular endothelial growth factor receptor-2 tyrosine kinase inhibitor for the treatment of cancer. Cancer Res. 2005, 65, 4389-4400.

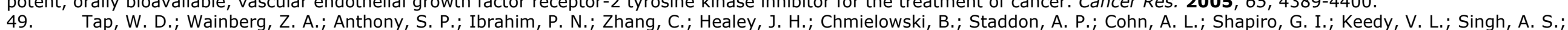

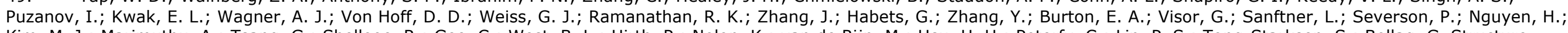

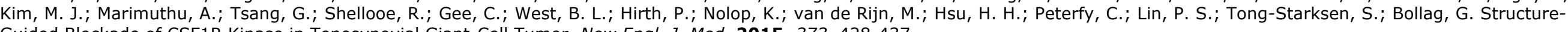
Guided Blockade of CSF1R Kinase in Tenosynovial Giant-Cell Tumor. New Engl. J. Med. 2015, 373, 428-437.

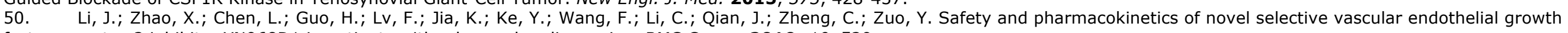

factor receptor-2 inhibitor YN968D1 in patients with advanced malignancies. BMC Cancer 2010, 10, 529.

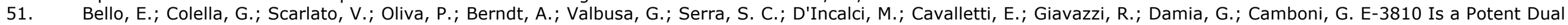
Inhibitor of VEGFR and FGFR that Exerts Antitumor Activity in Multiple Preclinical Models. Cancer Res. 2011, 71, 1396-1405.

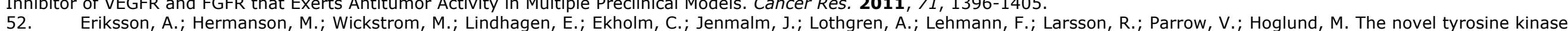

52. Eriksson, A.; Hermanson, M.; Wickstrom, M.; Lindhagen, E.; Ekholm, C.; Jenmalm, J.; Lothgren, A.; Lehmann, F.; Larsson, R.; Parrow, V.; Hogl
inhibitor AKN-028 has significant antileukemic activity in cell lines and primary cultures of acute myeloid leukemia. Blood Cancer Journal 2012, 2, e81.

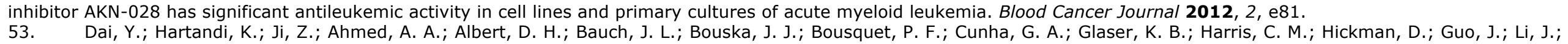

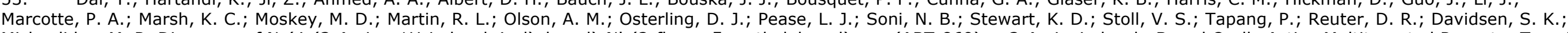

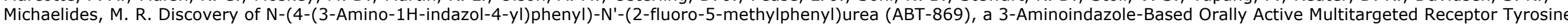
Kinase Inhibitor. J. Med. Chem. 2007, 50, 1584-1597.

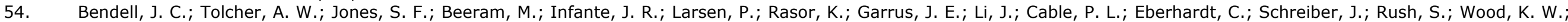

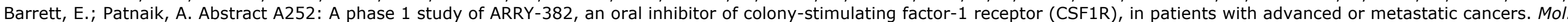
Cancer Ther. 2013,12, A252.

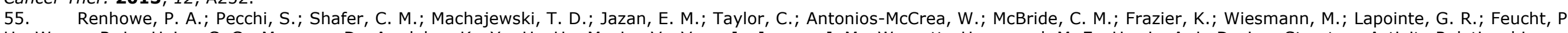

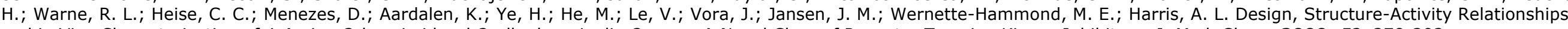
and in Vivo Characterization of 4-Amino-3-benzimidazol-2-ylhydroquinolin-2-ones: A Novel Class of Receptor Tyrosine Kinase Inhibitors. J. Med. Chem. 2009, 52, 278-292.

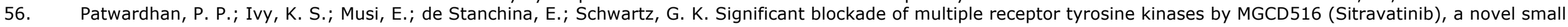

molecule inhibitor, shows potent anti-tumor activity in preclinical models of sarcoma. Oncotarget 2016, 7, 4093-4109.

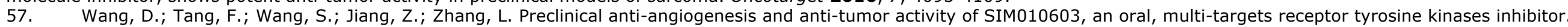

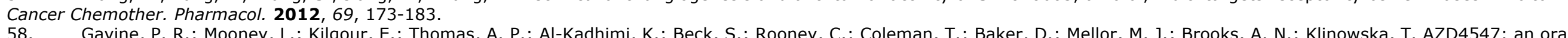

58. Gavine, P. R.; Mooney, L.; Kilgour, E.; Thomas, A. P.; Al-Kadhimi, K.; Beck, S.; Rooney, C.; Coleman, T.; Baker, D.; Mellor, M. J.; Brod
bioavailable, potent, and selective inhibitor of the fibroblast growth factor receptor tyrosine kinase family. Cancer Res. 2012, 72, $2045-2056$.

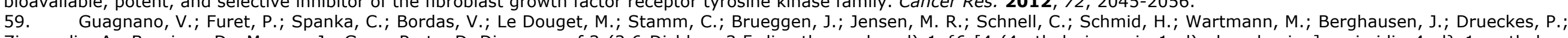

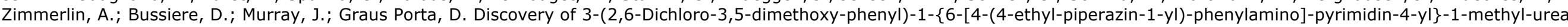
(NVP-BGJ398), A Potent and Selective Inhibitor of the Fibroblast Growth Factor Receptor Family of Receptor Tyrosine Kinase. J. Med. Chem. 2011, 54, 7066-7083.

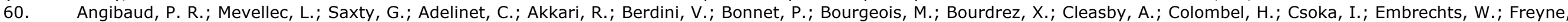

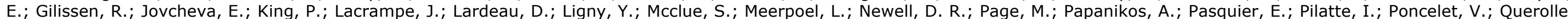

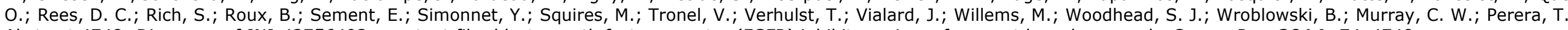
Abstract 4748: Discovery of JNJ-42756493, a potent fibroblast growth factor receptor (FGFR) inhibitor using a fragment based approach. Cancer Res. 2014, 74, 4748.

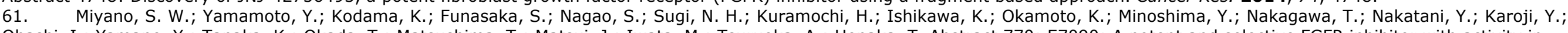

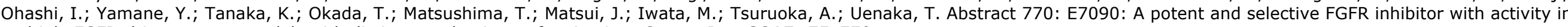
multiple FGFR-driven cancer models with distinct mechanisms of activation. Cancer Res. 2015, 75, 770.

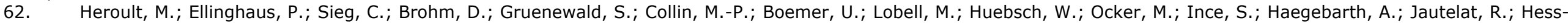
Stumpp, H.; Brands, M.; Ziegelbauer, K. Abstract 1739: Preclinical profile of BAY 1163877 - a selective pan-FGFR inhibitor in phase 1 clinical trial. Cancer Res. 2014, 74, 1739. 


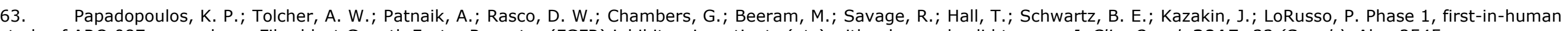
study of ARQ 087, an oral pan-Fibroblast Growth Factor Receptor (FGFR) inhibitor, in patients (pts) with advanced solid tumors. J. Clin. Oncol. 2015,33 (Suppl.), Abs. 2545.

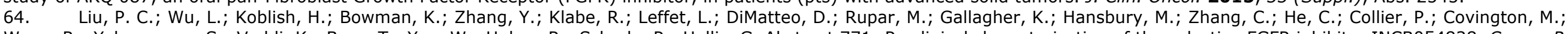

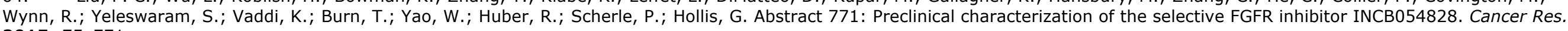
2015, 75, 771.

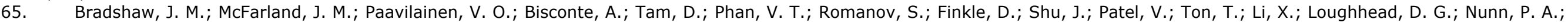

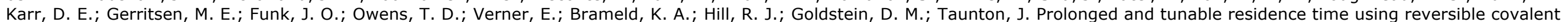
kinase inhibitors. Nat. Chem. Biol. 2015, 11, 525-531.

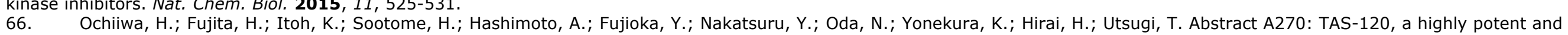

selective irreversible FGFR inhibitor, is effective in tumors harboring various FGFR gene abnormalities. Mol. Cancer Ther. 2013, 12, A270.

67. Gruellich, C. Cabozantinib: A MET, RET, and VEGFR2 Tyrosine Kinase Inhibitor. Recent Results in Cancer Research 2014, $201,207-214$.

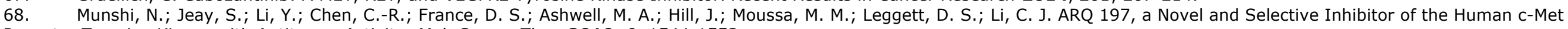
Receptor Tyrosine Kinase with Antitumor Activity. Mol. Cancer Ther. 2010, 9, 1544-1553.

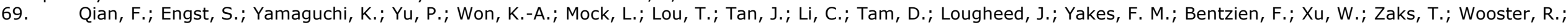

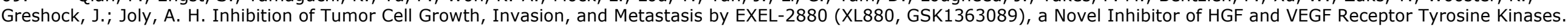

Cancer Res. 2009, 69, 8009-8016.

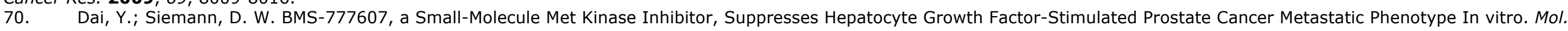

Cancer Ther. 2010, 9, 1554-1561.

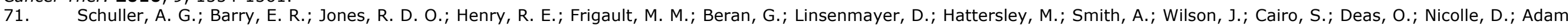

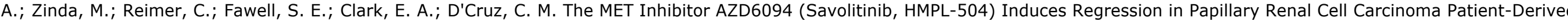
Xenograft Models. Clin. Cancer Res. 2015, 21, 2811-2819.

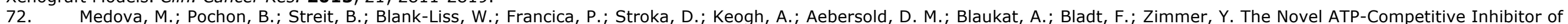

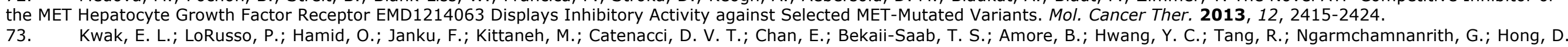

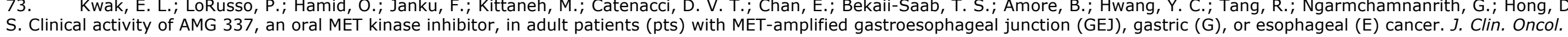
2015, 33 (Suppl. 3), Abs. 01 .

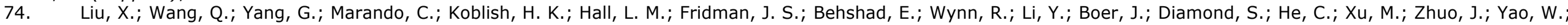

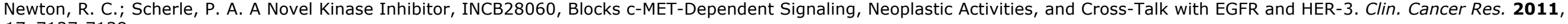
$17,7127-7138$.

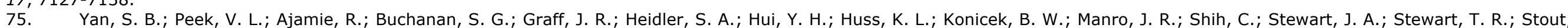

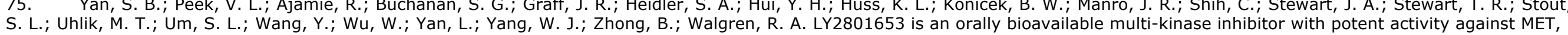
MST1R, and other oncoproteins, and displays anti-tumor activities in mouse xenograft models. Invest. New Drugs 2013, 31, 833-844.

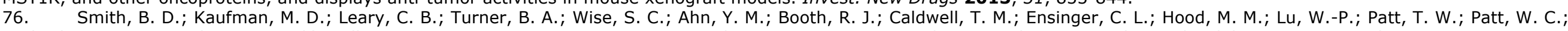

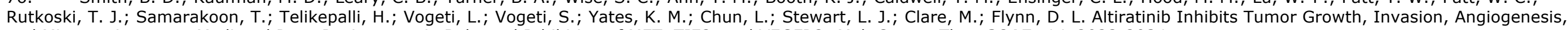
and Microenvironment-Mediated Drug Resistance via Balanced Inhibition of MET, TIE2, and VEGFR2. Mol. Cancer Ther. 2015, 14, 2023-2034.

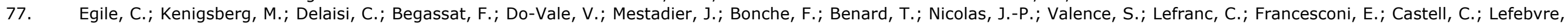

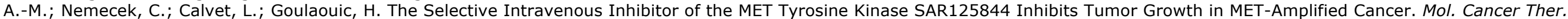
2015, 14, 384-394.

2015, 14, 384-394. $\quad$ Shaw, A. T.; Yasothan, U.; Kirkpatrick, P. Crizotinib. Nat. Rev. Drug Discov. 2011, 10, 897-898.

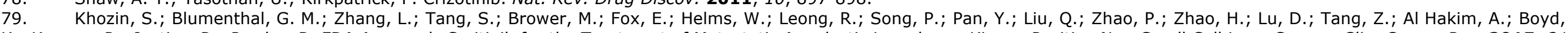

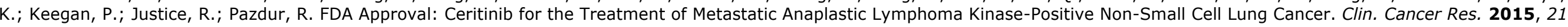
2436-2439.

80. McKeage, K. Alectinib: A Review of Its Use in Advanced ALK-Rearranged Non-Small Cell Lung Cancer. Drugs 2015, 75, 75-82.

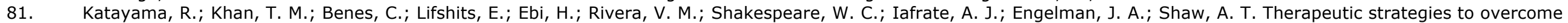

crizotinib resistance in non-small cell lung cancers harboring the fusion oncogene EML4-ALK. Proc. Natl. Adad. Sci. USA 2011, 108, 7535-7540.

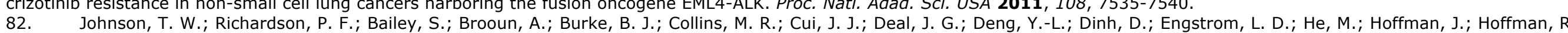

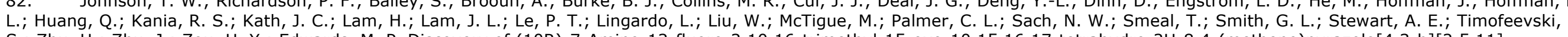

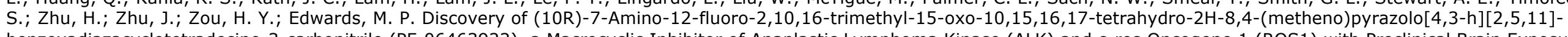

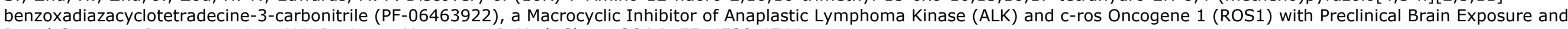
Broad-Spectrum Potency against ALK-Resistant Mutations. J. Med. Chem. 2014, 57, 4720-4744.

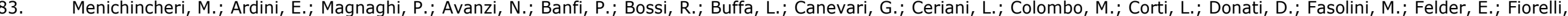

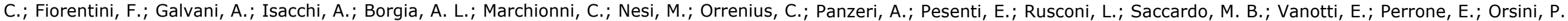




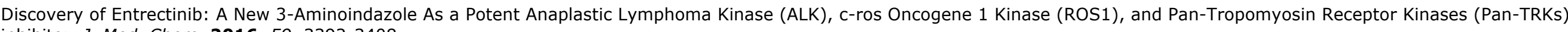
inhibitor. J. Med. Chem. 2016, 59, 3392-3408.

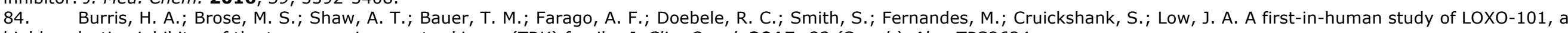
highly selective inhibitor of the tropomyosin receptor kinase (TRK) family. J. Clin. Oncol. 2015, 33 (Suppl.), Abs. TPS2624.

85. Myers, S. H.; Brunton, V. G.; Unciti-Broceta, A. AXL Inhibitors in Cancer: A Medicinal Chemistry Perspective. J. Med. Chem. $2016,59,3593-3608$.

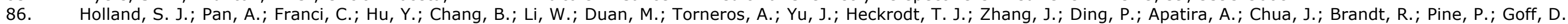

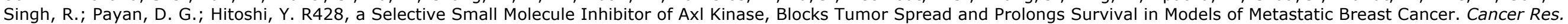

2010, 70, 1544-1554.

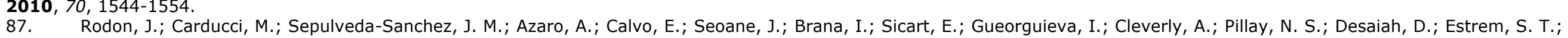

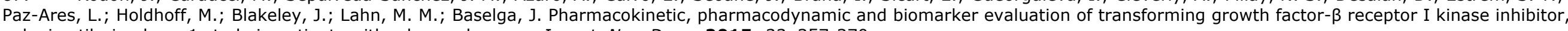
galunisertib, in phase 1 study in patients with advanced cancer. Invest. New Drugs 2015, 33, 357-370.

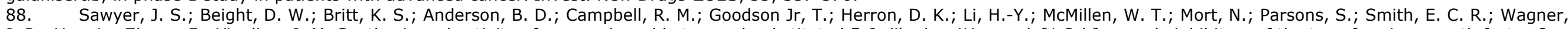

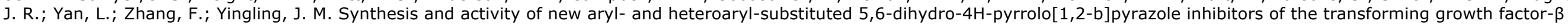
type I receptor kinase domain. Bioorg. Med. Chem. Lett. 2004, 14, 3581-3584.

89. Tefferi, A. JAK inhibitors for myeloproliferative neoplasms: clarifying facts from myths. Blood 2012, 119, 2721-2730.

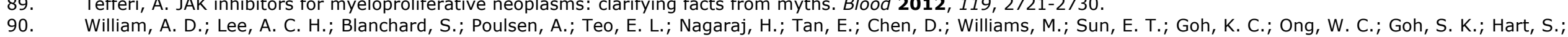

90. William, A. D.; Lee, A. C. H.; Blanchard, S.; Poulsen, A.; Teo, E. L.; Nagaraj, H.; Tan, E.; Chen, D.; Williams, M.; Sun, E. T.; Goh, K. C.; Ong, W. C.; Got
Jayaraman, R.; Pasha, M. K.; Ethirajulu, K.; Wood, J. M.; Dymock, B. W. Discovery of the Macrocycle 11-(2-Pyrrolidin-1-yl-ethoxy)-14,19-dioxa-5,7,26-triaza-

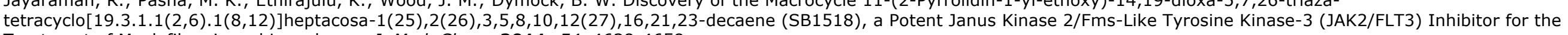
Treatment of Myelofibrosis and Lymphoma. J. Med. Chem. 2011, 54, 4638-4658.

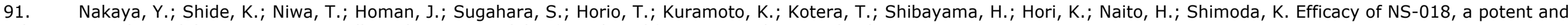
selective JAK2/Src inhibitor, in primary cells and mouse models of myeloproliferative neoplasms. Blood Cancer J. 2011, 1, e29.

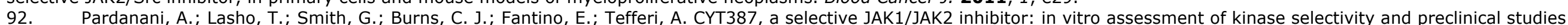

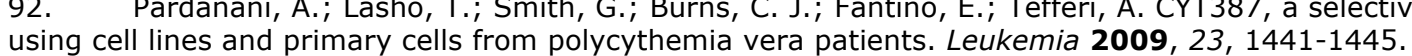

using cell lines and primary cells from polycythemia vera patients. Leukemia 2009, 23, 1441-1445.

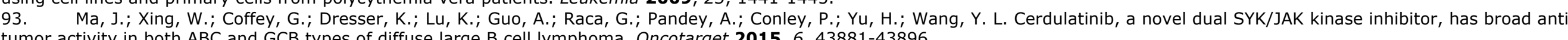

tumor activity in both $A B C$ and GCB types of diffuse large B cell lymphoma. Oncotarget 2015, 6, 43881-43896.

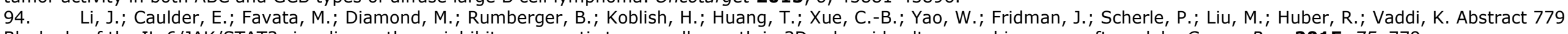

Blockade of the IL-6/JAK/STAT3 signaling pathway inhibits pancreatic tumor cell growth in 3D spheroid cultures and in xenograft models. Cancer Res. 2015, 75, 779.

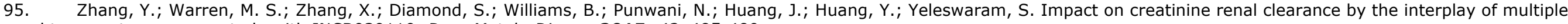
renal transporters: a case study with INCB039110. Drug Metab. Dispos. 2015, 43, 485-489.

96.

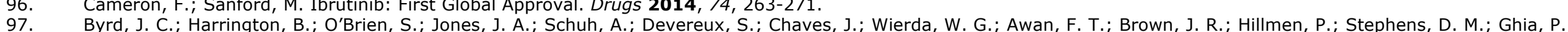

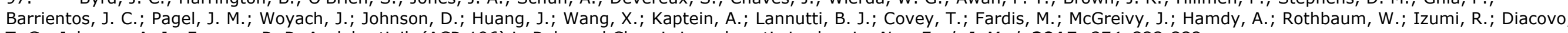
T. G.; Johnson, A. J.; Furman, R. R. Acalabrutinib (ACP-196) in Relapsed Chronic Lymphocytic Leukemia. New Engl. J. Med. 2015, 374, $323-332$.

98. Munakata, W. The development of novel Bruton's tyrosine kinase inhibitor, ONO-4059 for B-cell malignancies. Ketsueki Naika 2015, 71, 47-52.

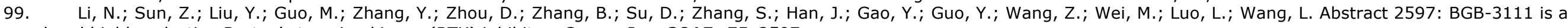
novel and highly selective Bruton's tyrosine kinase (BTK) inhibitor. Cancer Res. 2015, 75, 2597.

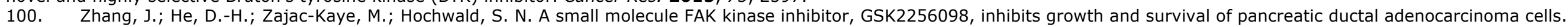

Cell Cycle 2014, $13,3143-3149$.
101 . Jones, S. F.; Siu, L. L.; Ben

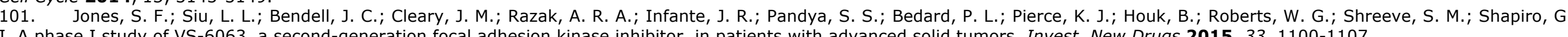

I. A phase I study of VS-6063, a second-generation focal adhesion kinase inhibitor, in patients with advanced solid tumors. Invest. New Drugs 2015, 33, 1100-1107.

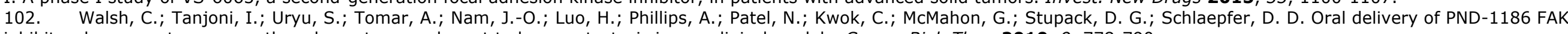

inhibitor decreases tumor growth and spontaneous breast to lung metastasis in pre-clinical models. Cancer Biol. Ther. 2010, 9, 778-790.

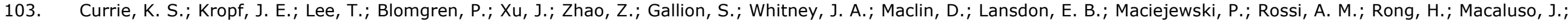

Barbosa, J.; Di Paolo, J. A.; Mitchell, S. A. Discovery of GS-9973, a Selective and Orally Efficacious Inhibitor of Spleen Tyrosine Kinase. J. Med. Chem. 2014, 57, 3856-3873.

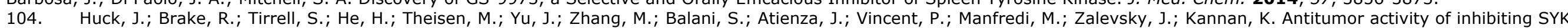

kinase with TAK-659, an investigational agent, in DLBCL models. J. Clin. Oncol. 2014, 32 (Suppl.), Abs. 8580.

105. Rini, B.; Kar, S.; Kirkpatrick, P. Temsirolimus. Nat. Rev. Drug Discov. 2007, 6, 599-600.

106. Atkins, M. B.; Yasothan, U.; Kirkpatrick, P. Everolimus. Nat. Rev. Drug Discov. 2009, 8, 535-536.

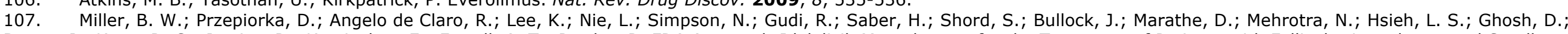

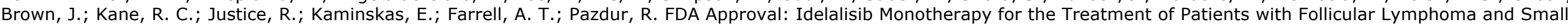

Lymphocytic Lymphoma. Clin. Cancer Res. 2015, 21, 1525-1529. 


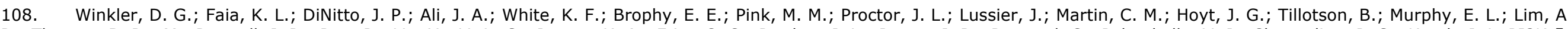

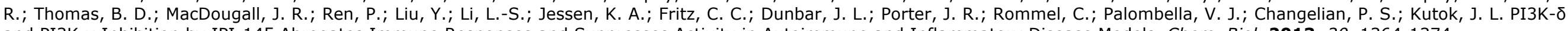
and PI3K-y Inhibition by IPI-145 Abrogates Immune Responses and Suppresses Activity in Autoimmune and Inflammatory Disease Models. Chem. Biol. 2013, $20,1364-1374$.

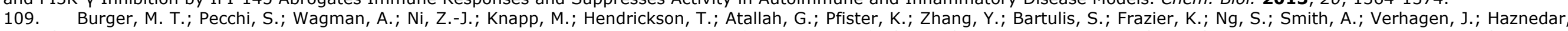

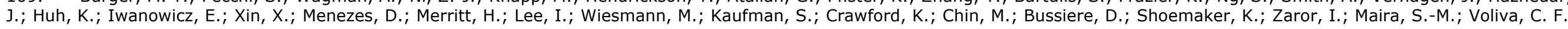
Identification of NVP-BKM120 as a Potent, Selective, Orally Bioavailable Class I PI3 Kinase Inhibitor for Treating Cancer. ACS Med. Chem. Lett. 2011, 2, 774-779.

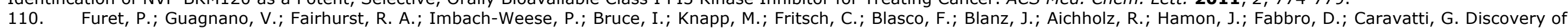
110. Furet, P.; Guagnano, V.; Fairhurst, R. A.; Imbach-Weese, P.; Bruce, I.; Knapp, M.; Fritsch, C.; Blasco, F.; Blanz, J.; Aichholz, R.; Hamon, J.; Fabbro, D.;
NVP-BYL719 a potent and selective phosphatidylinositol-3 kinase alpha inhibitor selected for clinical evaluation. Bioorg. Med. Chem. Lett. 2013, 23, 3741-3748.

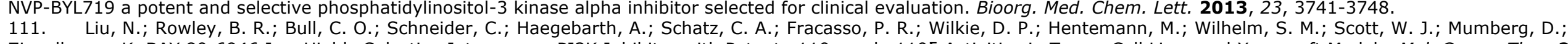

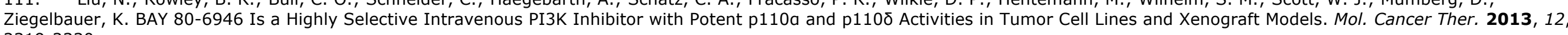
2319-2330.

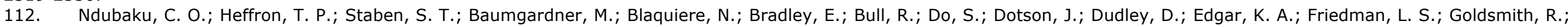

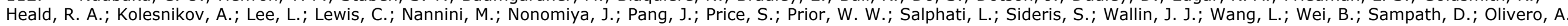

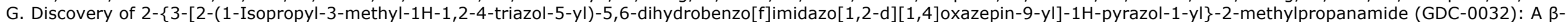
Sparing Phosphoinositide 3-Kinase Inhibitor with High Unbound Exposure and Robust in Vivo Antitumor Activity. J. Med. Chem. 2013, 56, 4597-4610.

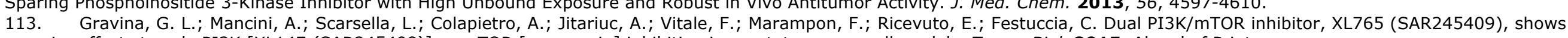
superior effects to sole PI3K [XL147 (SAR245408)] or mTOR [rapamycin] inhibition in prostate cancer cell models. Tumor Biol. 2015, Ahead of Print.

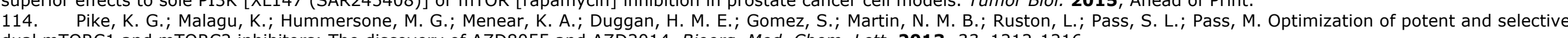

dual mTORC1 and mTORC2 inhibitors: The discovery of AZD8055 and AZD2014. Bioorg. Med. Chem. Lett. 2013, 23, 1212-1216.

115. Garlich, J. R.; De, P.; Dey, N.; Su, J. D.; Peng, X.; Miller, A.; Murali, R.; Lu, Y.; Mills, G. B.; Kundra, V.; Shu, H. K.; Peng, Q.; Durden, D. L. A Vascular Targeted Pan

Phosphoinositide 3-Kinase Inhibitor Prodrug, SF1126, with Antitumor and Antiangiogenic Activity. Cancer Res. 2008, 68, $206-215$.

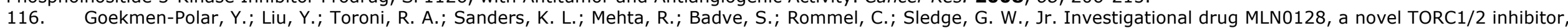

demonstrates potent oral antitumor activity in human breast cancer xenograft models. Breast Cancer Res. Treat. 2012, 136, 673-682.

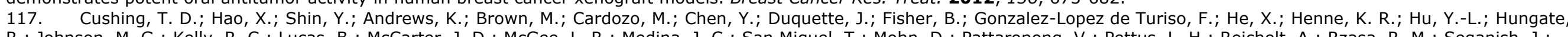

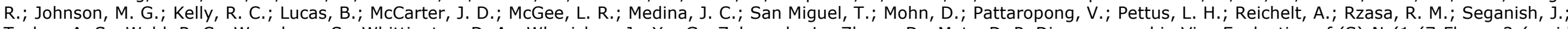

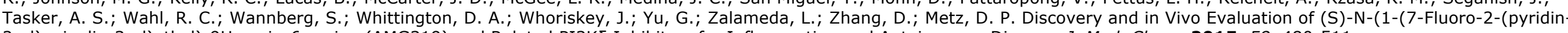
2-yl)quinolin-3-yl)ethyl)-9H-purin-6-amine (AMG319) and Related PI3Kס Inhibitors for Inflammation and Autoimmune Disease. J. Med. Chem. 2015, 58, 480-511.

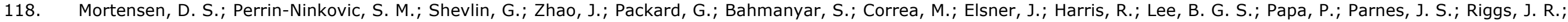

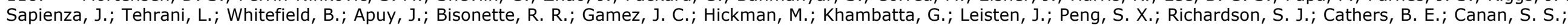

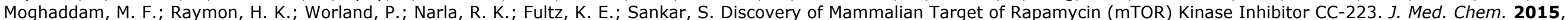
$58,5323-5333$.

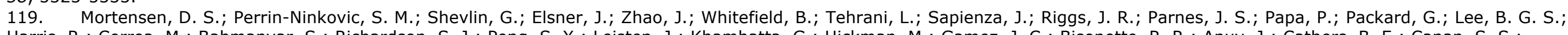

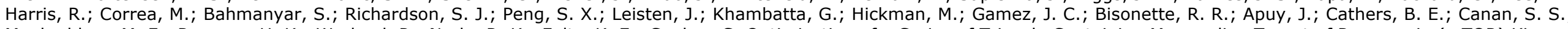

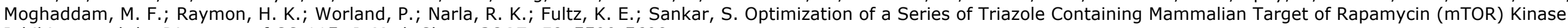
Inhibitors and the Discovery of CC-115. J. Med. Chem. 2015, 58, 5599-5608.

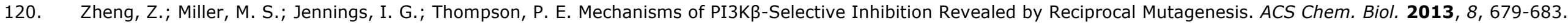

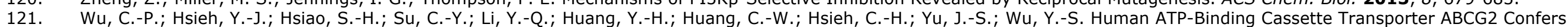
Resistance to CUDC-907, a Dual Inhibitor of Histone Deacetylase and Phosphatidylinositol 3-Kinase. Mol. Pharmaceutics 2016, 13, 784-794.

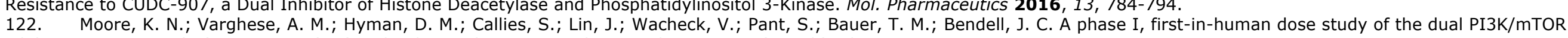

122. Moore, K. N.; Varghese, A. M.; Hyman, D. M.; Callies, S.; Lin, J.; Wacheck, V.; Pant, S.; Bauer, T. M.; Be
inhibitor LY3023414 (LY) in patients (pts) with advanced cancer. J. Clin. Oncol. 2015, 33 (Suppl.), Abs. 11075.

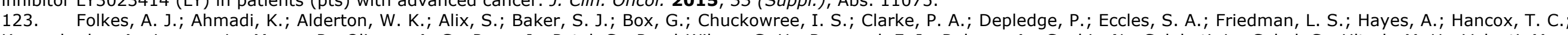

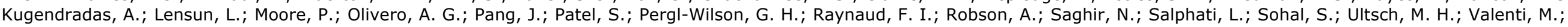

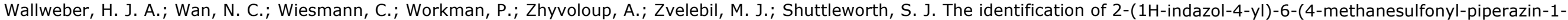

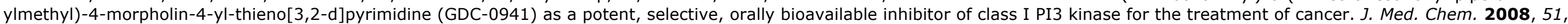
$5522-5532$.

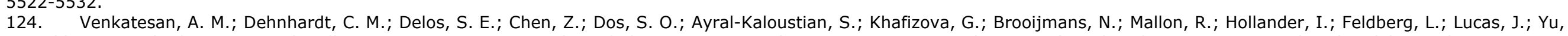

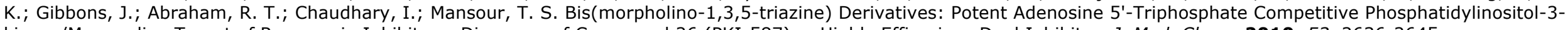
kinase/Mammalian Target of Rapamycin Inhibitors: Discovery of Compound 26 (PKI-587), a Highly Efficacious Dual Inhibitor. J. Med. Chem. 2010, 53, 2636-2645.

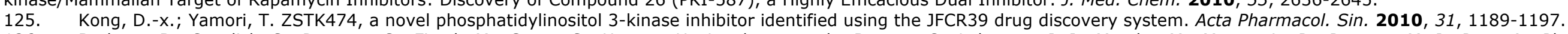

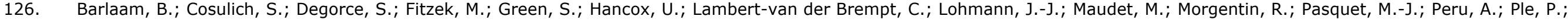

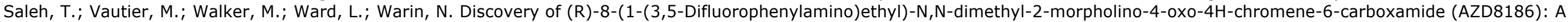
Potent and Selective Inhibitor of PI3K $\beta$ and PI3Kס for the Treatment of PTEN-Deficient Cancers. J. Med. Chem. 2015, 58, $943-962$. 


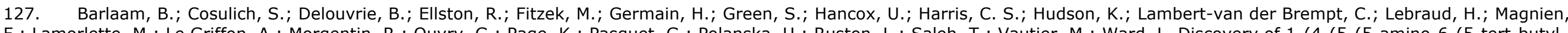

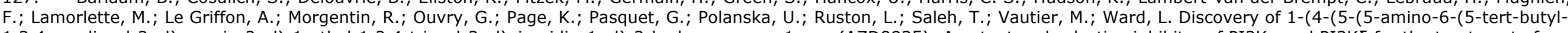

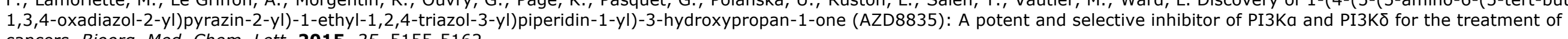
cancers. Bioorg. Med. Chem. Lett. 2015, 25, 5155-5162.

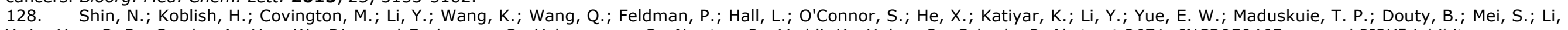

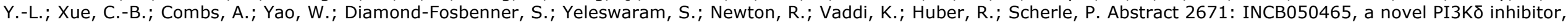
synergizes with PIM protein kinase inhibition to cause tumor regression in a model of DLBCL. Cancer Res. 2015, 75, 2671.

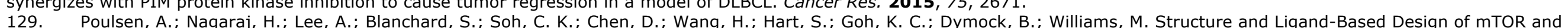

129. Poulsen, A.; Nagaraj, H.; Lee, A.; Blanchard, S.; Soh, C. K.; Chen, D.; Wang, H.; Hart, S.; Goh, K. C.; Dymock, B.; Williams,
PI3-Kinase Inhibitors Leading to the Clinical Candidates VS-5584 (SB2343) and SB2602. J. Chem. Inf. Model. 2014, 54, 3238-3250.

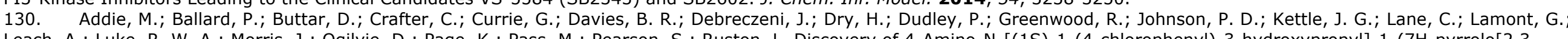

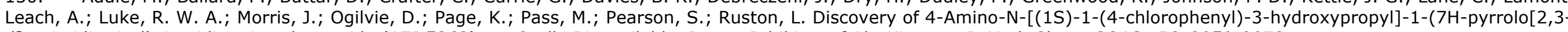

d]pyrimidin-4-yl)piperidine-4-carboxamide (AZD5363), an Orally Bioavailable, Potent Inhibitor of Akt Kinases. J. Med. Chem. 2013, 56, 2059-2073.

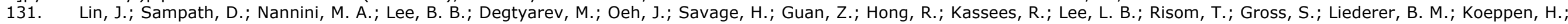

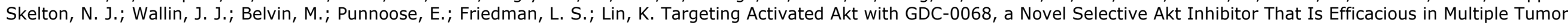
Models. Clin. Cancer Res. 2013, 19, 1760-1772.

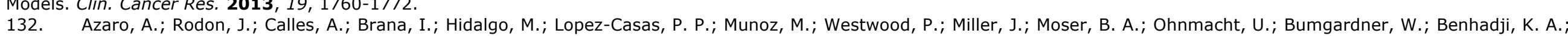

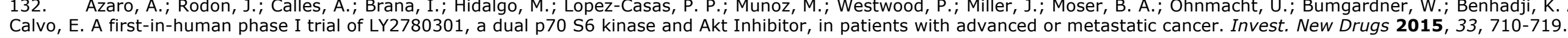

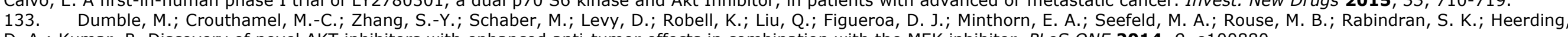

D. A.; Kumar, R. Discovery of novel AKT inhibitors with enhanced anti-tumor effects in combination with the MEK inhibitor. PLOS ONE 2014, 9, e100880.

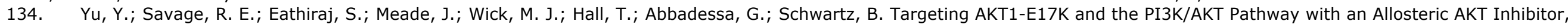

ARQ 092. PLOS ONE 2015, 10, e0140479.

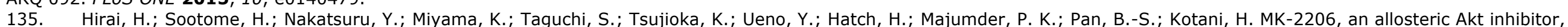
enhances antitumor efficacy by standard chemotherapeutic agents or molecular targeted drugs in vitro and in vivo. Mol. Cancer Ther. 2010, 9, $1956-1967$.

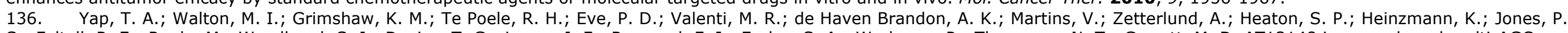

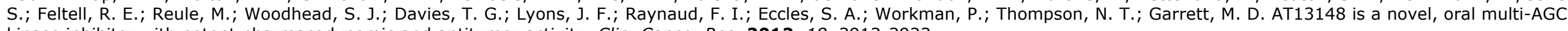
kinase inhibitor with potent pharmacodynamic and antitumor activity. Clin. Cancer Res. 2012, 18, 3912-3923.

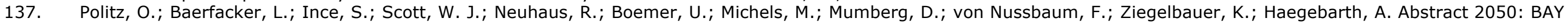

1125976, a highly selective and potent allosteric AKT1/2 inhibitor, for the treatment of cancers with aberrations in the PI3K-AKT-mTOR pathway. Cancer Res. 2013, 73, 2050.

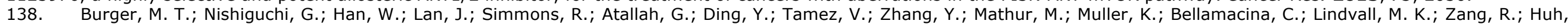

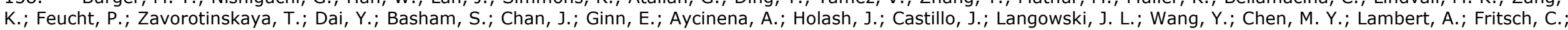

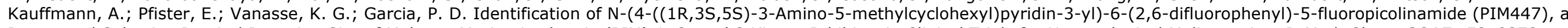

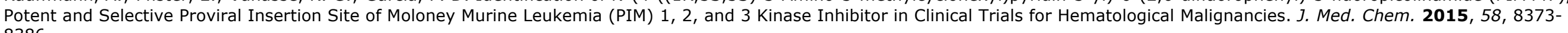
8386.

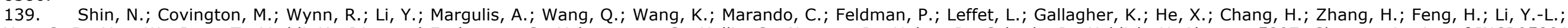

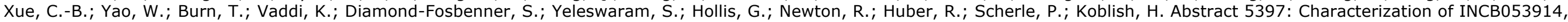
a novel pan-PIM kinase inhibitor. Cancer Res. 2015, 75, 5397.

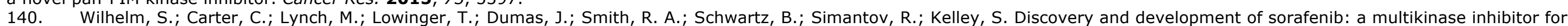
treating cancer. Nat. Rev. Drug Discov. 2006, 5, 835-844.

treating cancer. Nat. Rev. Drug Discov. 2006, 5, 835-844.
141. Flaherty, K. T.; Yasothan, U.; Kirkpatrick, P. Vemurafenib. Nat. Rev. Drug Discov. 2011, 10, 811-812.

141. Flaherty, K. T.; Yasothan, U.; Kirkpatrick, P. Vemurafenib. Nat. Rev. Drug Discov. 2011, 10, 811-812.
142. Ballantyne, A. D.; Garnock-Jones, K. P. Dabrafenib: First Global Approval. Drugs 2013, 73, 1367-1376.

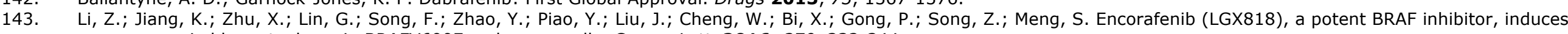
senescence accompanied by autophagy in BRAFV600E melanoma cells. Cancer Lett. 2016, 370, 332-344.

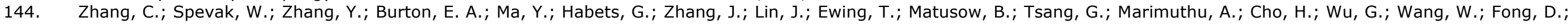

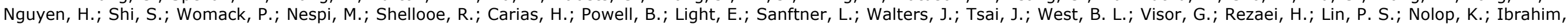
P. N.; Hirth, P.; Bollag, G. RAF inhibitors that evade paradoxical MAPK pathway activation. Nature 2015, 526, 583-586.

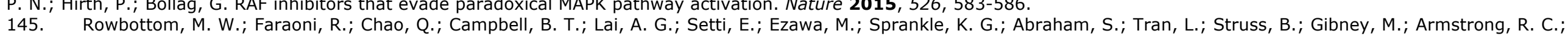

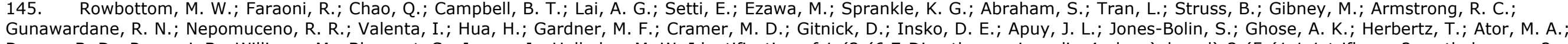

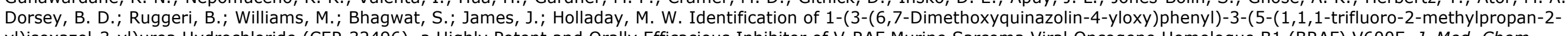

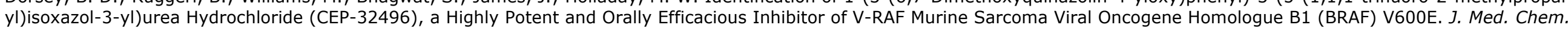

2012, 55, 1082-1105. 


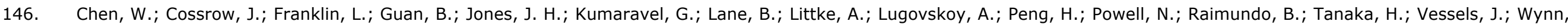

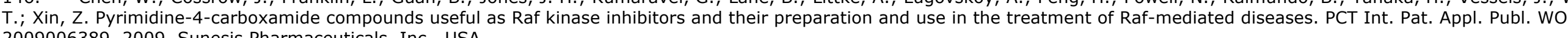
2009006389, 2009, Sunesis Pharmaceuticals, Inc., USA

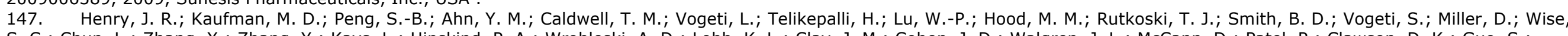

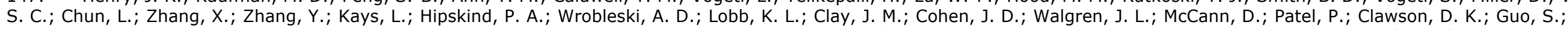

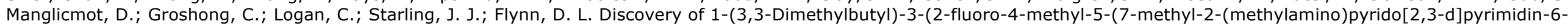

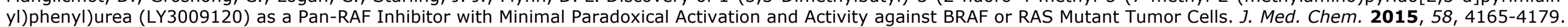

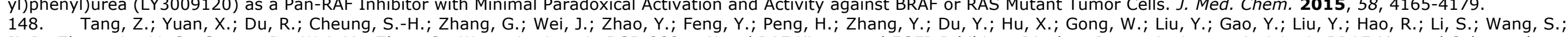

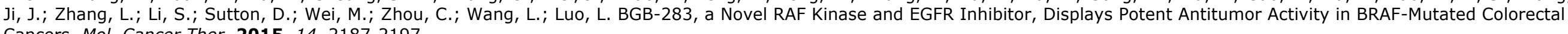
Cancers. Mol. Cancer Ther. 2015, 14, 2187-2197.

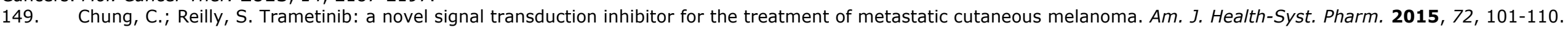

150. Garnock-Jones, K. P. Cobimetinib: First Global Approval. Drugs 2015, 75, 1823-1830.

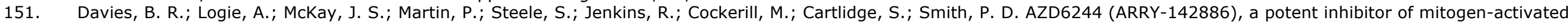

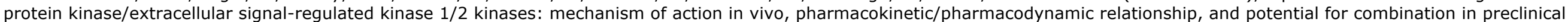
models. Mol. Cancer Ther. 2007, 6, 2209-2219.

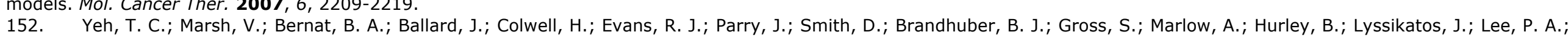

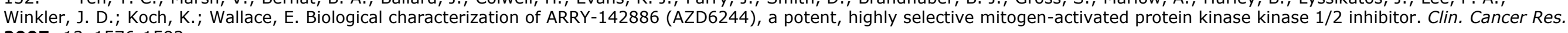
2007, 13, 1576-1583.

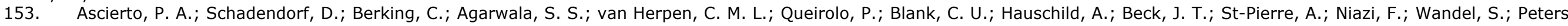

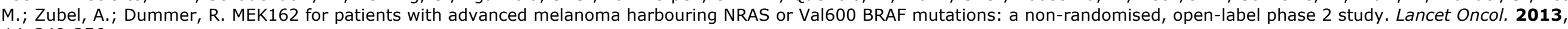
$14,249-256$.

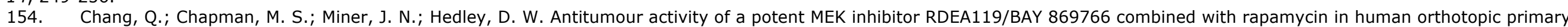
pancreatic cancer xenografts. BMC Cancer 2010, 10,515.

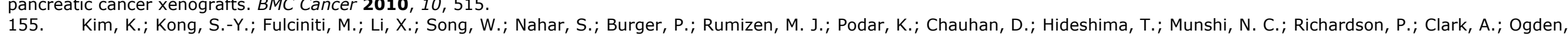

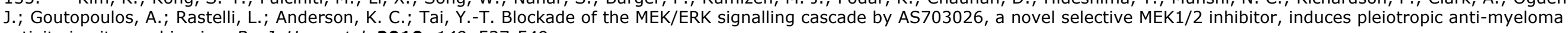
activity in vitro and in vivo. Br. J. Haematol. 2010, 149, 537-549.

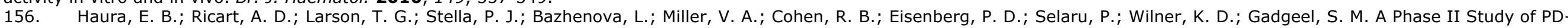
0325901, an Oral MEK Inhibitor, in Previously Treated Patients with Advanced Non-Small Cell Lung Cancer. Clin. Cancer Res. 2010, 16, $2450-2457$.

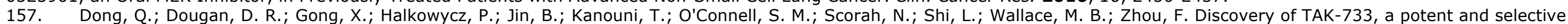
MEK allosteric site inhibitor for the treatment of cancer. Bioorg. Med. Chem. Lett. 2011, 21, 1315-1319.

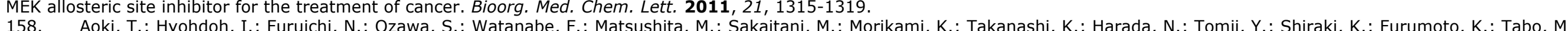

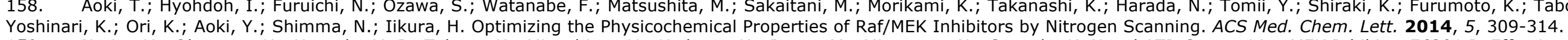

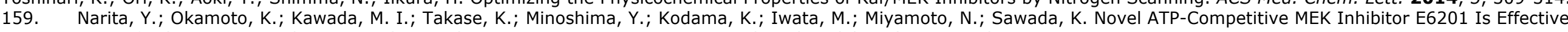
against Vemurafenib-Resistant Melanoma Harboring the MEK1-C121S Mutation in a Preclinical Model. Mol. Cancer Ther. 2014, 13, 823-832.

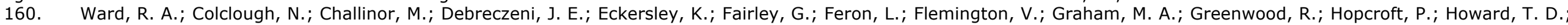

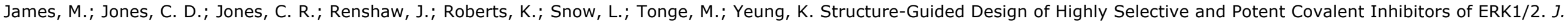
Med. Chem. 2015, 58, 4790-4801.

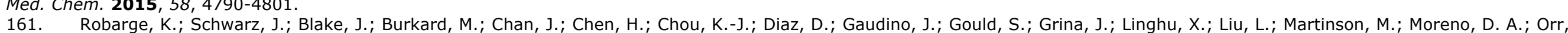

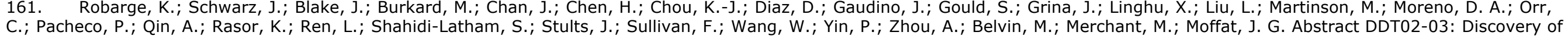
GDC-0994, a potent and selective ERK1/2 inhibitor in early clinical development. Cancer Res. 2014, 74, DDT02-03.

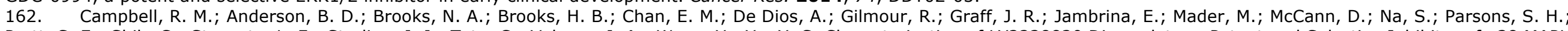

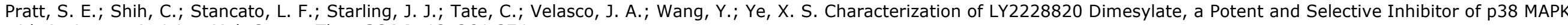
with Antitumor Activity. Mol. Cancer Ther. 2014, 13, 364-374.

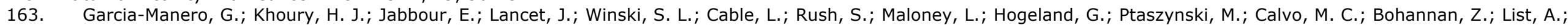

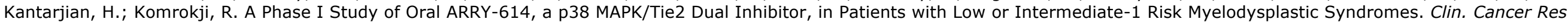
2015, 21, 985-994.

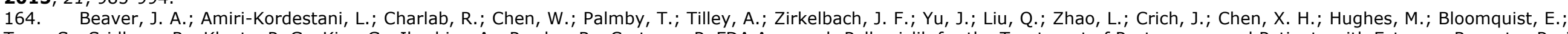

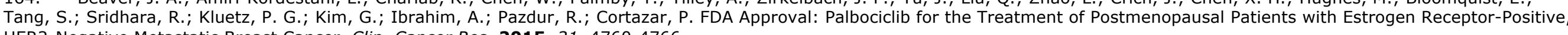
HER2-Negative Metastatic Breast Cancer. Clin. Cancer Res. 2015, 21, 4760-4766.

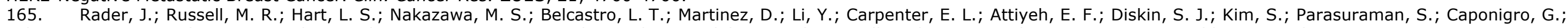

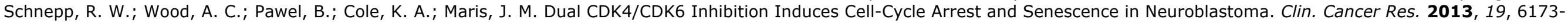
6182. 
166. Tate, S. C.; Cai, S.; Ajamie, R. T.; Burke, T.; Beckmann, R. P.; Chan, E. M.; De Dios, A.; Wishart, G. N.; Gelbert, L. M.; Cronier, D. M. Semi-Mechanistic

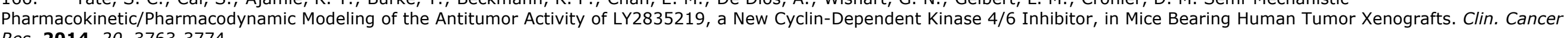
Res. 2014, 20, 3763-3774.

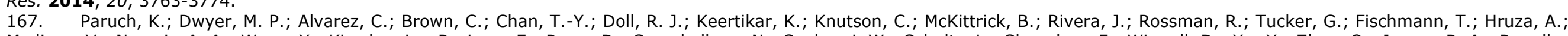

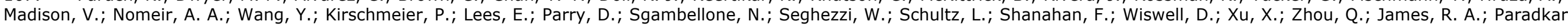

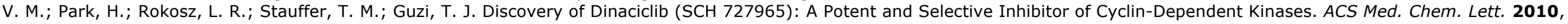
$1,204-208$.

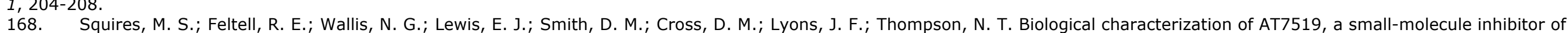
168. Squires, M. S.; Feltell, R. E.; Wallis, N. G.; Lewis, E. J.; Smith, D. M.; Cross, D. M.;
cyclin-dependent kinases, in human tumor cell lines. Mol. Cancer Ther. 2009, 8, 324-332.

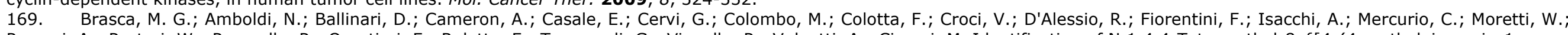

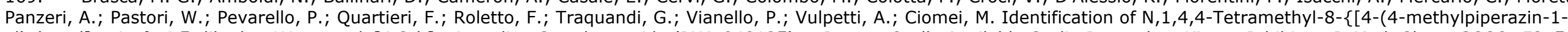

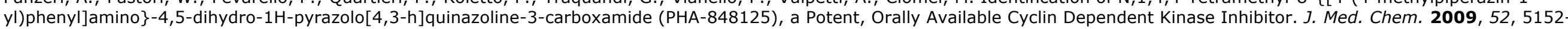
5163.

170. Zeidner, J. F.; Karp, J. E. Clinical activity of alvocidib (flavopiridol) in acute myeloid leukemia. Leuk. Res. 2015, 39, 1312-1318.

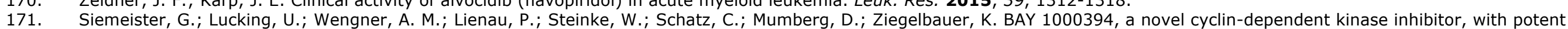
171. Siemeister, G.; Lucking, U.; Wengner, A. M.; Lienau, P.; Steinke, W.; Schatz, C.; Mumberg, D.; Ziegelbauer, K. BAY
antitumor activity in mono- and in combination treatment upon oral application. Mol. Cancer Ther. 2012, 11, 2265-2273.

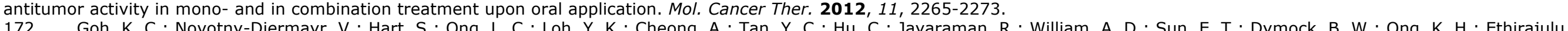
172. Goh, K. C.; Novotny-Diermayr, V.; Hart, S.; Ong, L. C.; Loh, Y. K.; Cheong, A.; Tan, Y. C.; Hu, C.; Jayaraman, R.; William, A. D.; Sun, E. T.; Dymock, B. W.
K.; Burrows, F.; Wood, J. M. TG02, a novel oral multi-kinase inhibitor of CDKs, JAK2 and FLT3 with potent anti-leukemic properties. Leukemia 2012, 26 , $236-243$.

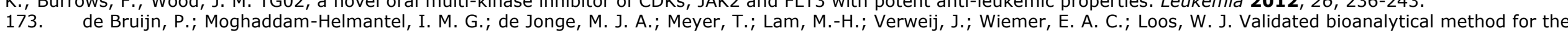

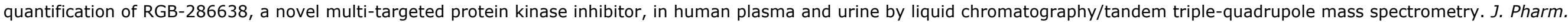
Biomed. Anal. 2009, 50, 977-982.

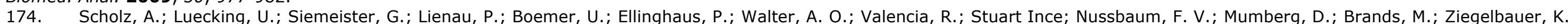

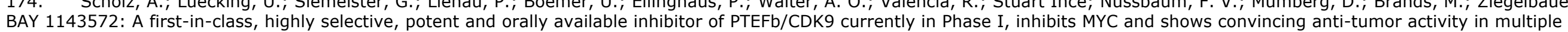
BAY 1143572: A first-in-class, highly selective, potent and orally available inhibitor of PTEFb/CDK9 currently in Phase I, inhibits MYC and shows convincing anti-tumor activity
xenograft models by the induction of apoptosis. In 106th Annual Meeting of the American Association for Cancer Research, Philadelphia, PA, USA, $2015 ;$ pp Abs. DDT02-02.

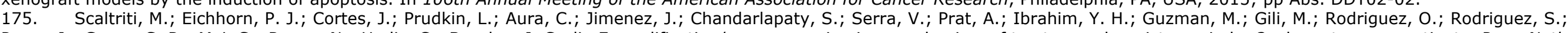

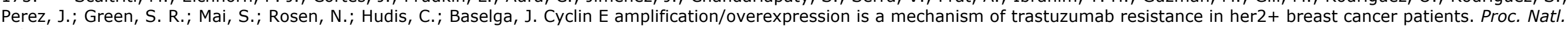
Adad. Sci. USA 2011, 108, 3761-3766.

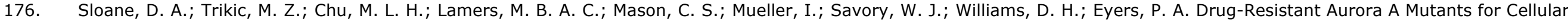
Target Validation of the Small Molecule Kinase Inhibitors MLN8054 and MLN8237. ACS Chem. Biol. 2010, 5, 563-576.

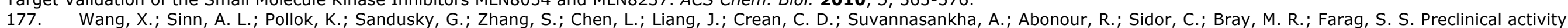
of a novel multiple tyrosine kinase and aurora kinase inhibitor, ENMD-2076, against multiple myeloma. Br. J. Haematol. 2010, 150, $313-325$.

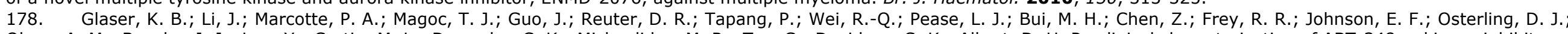

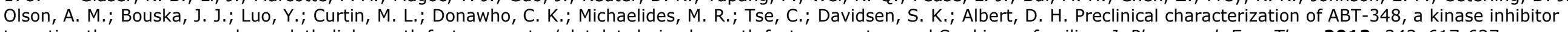
targeting the aurora, vascular endothelial growth factor receptor/platelet-derived growth factor receptor, and Src kinase families. J. Pharmacol. Exp. Ther. 2012, 343, 617-627.

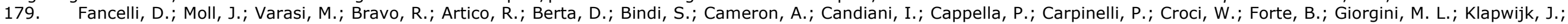

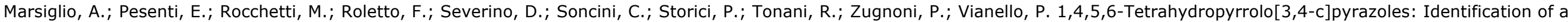
Potent Aurora Kinase Inhibitor with a Favorable Antitumor Kinase Inhibition Profile. J. Med. Chem. 2006, 49, 7247-7251.

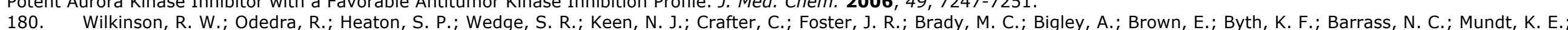

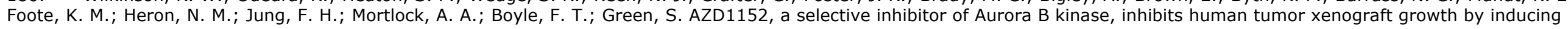
apoptosis. Clin. Cancer Res. 2007, 13, 3682-3688.

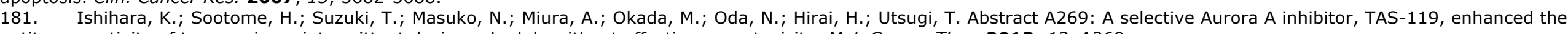
antitumor activity of taxanes in an intermittent dosing schedule without affecting neurotoxicity. Mol. Cancer Ther. 2013, 12, A269.

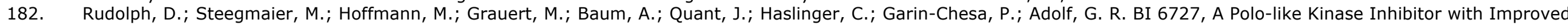
Pharmacokinetic Profile and Broad Antitumor Activity. Clin. Cancer Res. 2009, 15, 3094-3102.

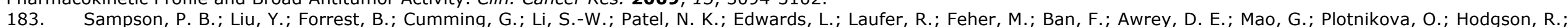

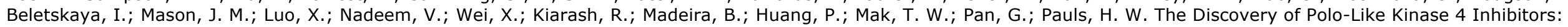

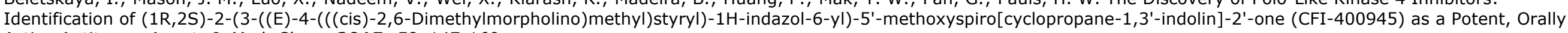
Active Antitumor Agent. J. Med. Chem. 2015, 58, 147-169.

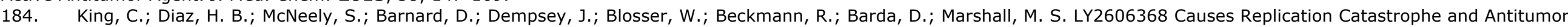
Effects through CHK1-Dependent Mechanisms. Mol. Cancer Ther. 2015, 14, 2004-2013.

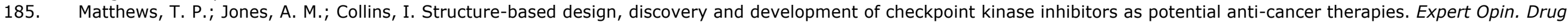
Discov. 2013, 8, 621-640. 


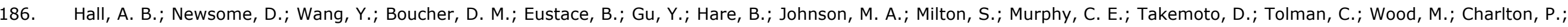

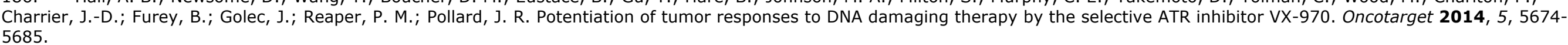

187. Karnitz, L. M.; Zou, L. Molecular Pathways: Targeting ATR in Cancer Therapy. Clin. Cancer Res. 2015, 21, 4780-4785.

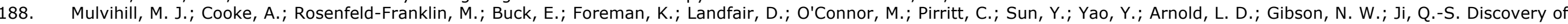
OSI-906: a selective and orally efficacious dual inhibitor of the IGF-I receptor and insulin receptor. Future Med. Chem. 2009, 1, 1153-1171.

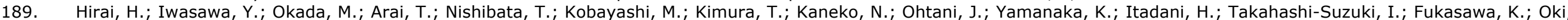

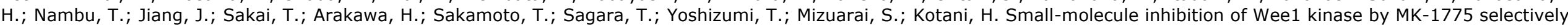
sensitizes p53-deficient tumor cells to DNA-damaging agents. Mol. Cancer Ther. 2009, 8, 2992-3000.

190. Pierre, F.; Chua, P. C.; O'Brien, S. E.; Siddiqui-Jain, A.; Bourbon, P.; Haddach, M.; Michaux, J.; Nagasawa, J.; Schwaebe, M. K.; Stefan, E.; Vialettes, A.; Whitten, J. P.; Darjania, L.; Stansfield, R.; Anderes, K.; Bliesath, J.; Drygin, D.; Ho, C.; Omori, M.; Proffitt, C.; Streiner, N.; Trent, K.; Rice, W. G.; Ryckman, D. M. Discovery and SAR of 5-(3654.

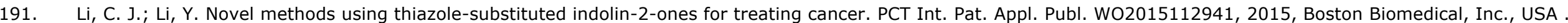

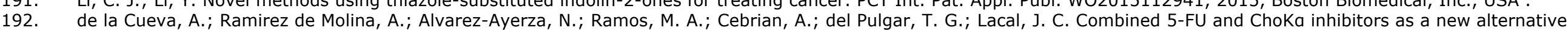
therapy of colorectal cancer: evidence in human tumor-derived cell lines and mouse xenografts. PLoS ONE 2013, 8, e64961.

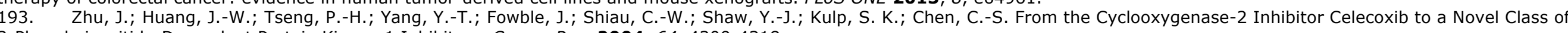
3-Phosphoinositide-Dependent Protein Kinase-1 Inhibitors. Cancer Res. 2004, 64, 4309-4318.

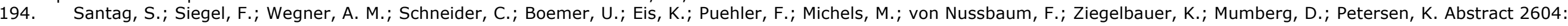
Preclinical anti-tumor efficacy and mode of action of a novel, orally available, selective MKNK1 inhibitor [BAY 1143269]. Cancer Res. 2015, 75, 2604.

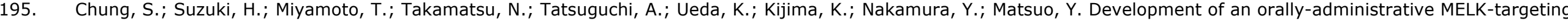
inhibitor that suppresses the growth of various types of human cancer. Oncotarget 2012, 3, 1629-1640. 